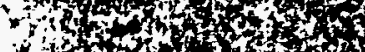

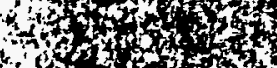

$430,4+2$

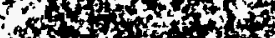

$4+4+4$

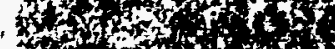

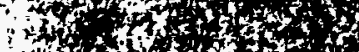

$7 y+2 x+4 x^{2}$

4 a

ons

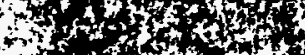

ond ond

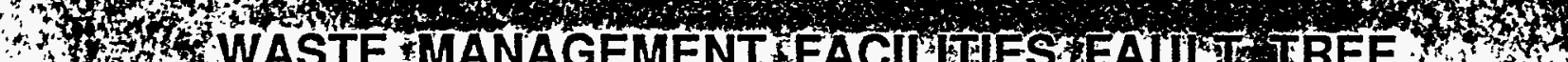

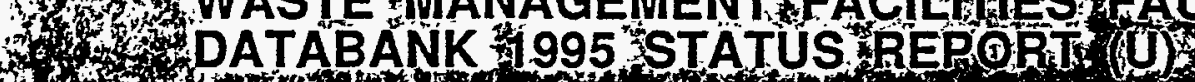

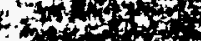

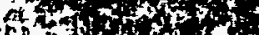

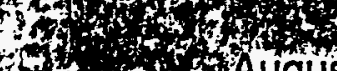

2t

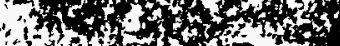

B

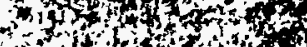

Wustow W. Ninnick

H. W. Welliaker

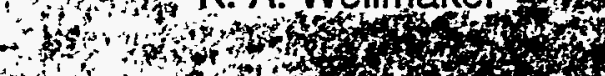

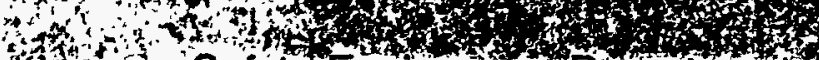

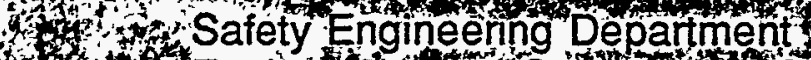

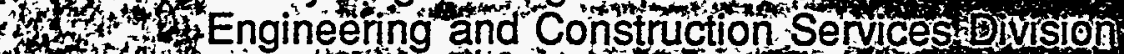

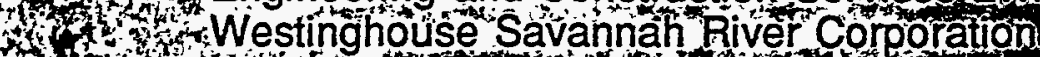

A

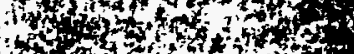

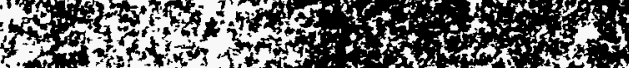

Hat

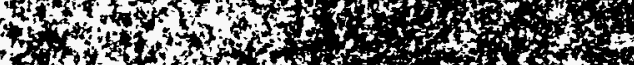

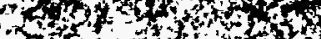

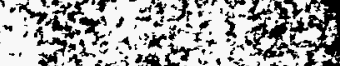

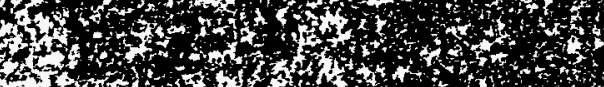

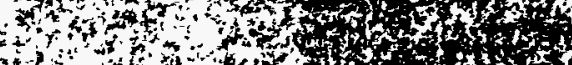

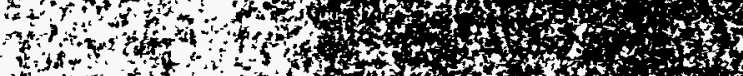

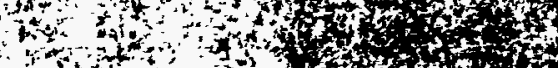

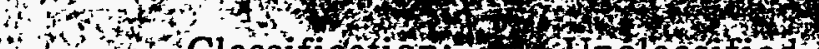

Classification ${ }^{2}$, nolassified

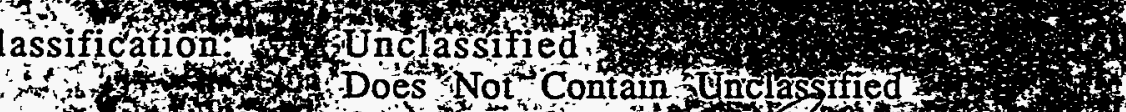

Retention

Eifetime

Gatabanks:

inclidents.

Biskissessment

tot

(4)

t.

tits

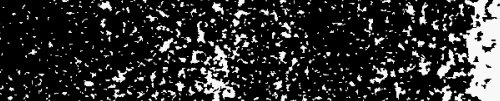

and controlled Nuclear on formation

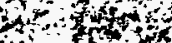

Authorized Derivative Classifier:

RREE

and Reviewing Official y

sition

10

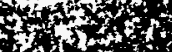

atis

(2) $6,+2$

thy

rithor

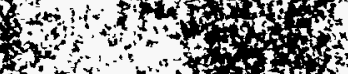

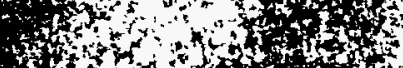

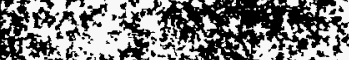

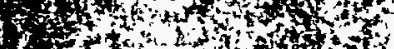

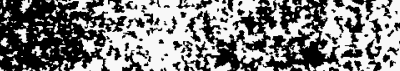

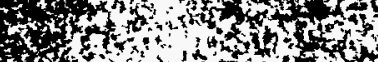

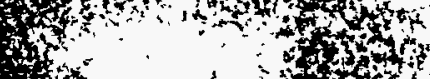

4 and

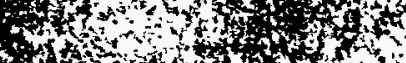

4 , now

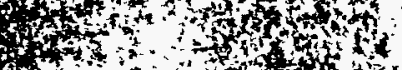

(x)

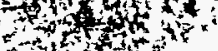

as

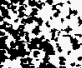

(2)

$x^{2}$

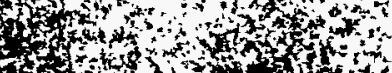

13, +2

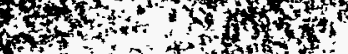

$4 \mathrm{~s}^{4}$

fint

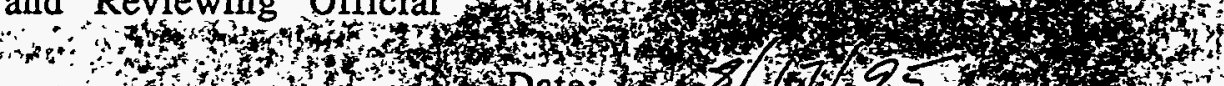

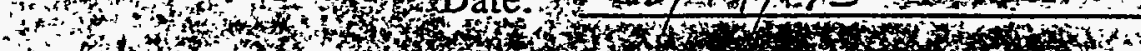

atom,

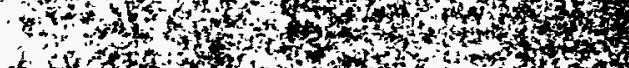

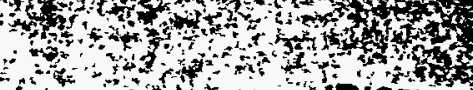

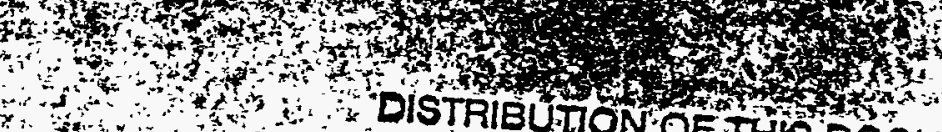

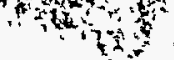

L

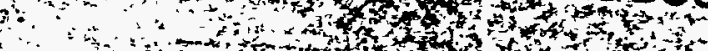






\section{Waste Management Facilities Fault Tree Databank 1995 Status Report (U)}

by

K. A. Wellmaker

Westinghouse Savannah River Company

Savannah River Site

Aiken, South Carolina 29808

W. V. Minnick

\section{MASTER}

This paper was prepared in connection with work done under the above contract number with the U.S.

Department of Energy. By acceptance of this paper, the publisher and/or recipient acknowledges the U.S.

Government's right to retain a nonexclusive, royalty-free license in and to any copyright covering this paper, along with the right to reproduce and to authorize others to reproduce all or part of the copyrighted paper. 
TO: $\quad$ Status Report Recipient

FROM: W.V. Minnick

Attached is a copy of the Waste Management Fault Tree Data Bank 1995 Status Report. The report contains the revised listing of the Area, Source, Facility, Operation, and Equipment codes to be used in conjunction with 'Risk Assessment Methodology's Fault Tree Data Banks User's Guide' to extract information from the data bank.

The revised listing represents a culmination of internal reviews and user surveys to improve databank operations. The user interface was improved with the addition of revised alpha numeric operation codes and a categorized equipment code listing which simplifies search code selection. Approximately 300 Waste Management Equipment Codes were added to minimize future additions, permit more specific search capability and reduce the need for manual review of large volume dumps. The databank was recoded (35,000 entries) to ensure uniform application of the revised codes and remove limitations imposed by previous codes.

The recoding effort has taken 12 months. During this time some 1993, all 1994 and all issued 1995 incidents were coded but backlogged for entry. It is anticipated the backlog will be worked off during the next 9 to 12 months. concurrent with entry of current data.

Safety Information Management Analysis data bank personnel are located in Centennial Corporate Center, Building 992-1W. Databank personnel are ready to help you with all your data needs: including data analysis. For more information on the SIMA data banks please contact W.V. Minnick, ext. 4-5174 or K.A. Wellmaker, ext. 4-5087. 

PROJECT: $\quad$ WM Databank Status Report

DOCUMENT: $\quad$ WSRC-TR-95-0173

TITLE:

Waste Management Facilities Fault Tree Databank

(WM) 1995 Status Report

APPROVALS

PDot

D. J. Baker, Manager, SIMA Group

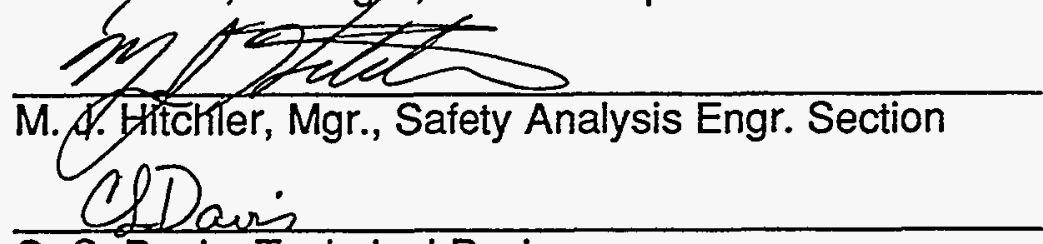

C. S. Davis, Technical Reviewer
$8 / 21 / 95$

Date

$\frac{8 / 25 / 55}{\text { bate }}$

$8 / 21 / 95$

Date 


\section{WASTE MANAGEMENT FAULT TREE DATABANK (WM) 1995 STATUS REPORT (U)}

\section{INTRODUCTION}

The Safety Information Management and Analysis Group (SIMA) of the Safety Engineering Department (SED) maintains compilations of incidents that have occurred in the Separations and Process Control, Waste Management, Fuel Fabrication, Tritium and SRTC facilities. This report records the status of the Waste Management (WM) Databank at the end of CY-1994. The WM Databank contains more than 35,000 entries ranging from minor equipment malfunctions to incidents with significant potential for injury or contamination of personnel. This report documents the status of the WM Databank including the availability, training, sources of data, search options, Quality Assurance, and usage to which these data have been applied. Periodic updates to this memorandum are planned as additional data or applications are acquired.

In June 1992, the Waste Management incidents were removed from the Separation and Process Control Databank (SEPR) and incorporated in the Waste Management Databank. Since its inception, 440 requests for data have been processed for use outside the SIMA group. The number of data searches performed by others is not recorded. Data searches performed for others by SIMA are tabulated in Table 1.

The Safety Information Management \& Analysis Group is here to assist in searching the databanks as well as data analysis. Coding assigned to incidents and events are included in this report however, our experience with the data entry and searching for historical data has proven to be a valuable asset available to you. If you do access the databanks directly, please keep in mind that if you plan to use information from the databanks to generate Level 1 calculations or modify Technical Baselines you must:

- be sure the search codes you use will retrieve a complete data set

- be sure that the date range you use will ensure that the source documents were available for the search category

- be sure to remove duplicates that may have been reported from more than one source

- be sure that each entry is appropriate for the analysis

SIMA-is prepared to provide you with results from the databanks rather than raw data. Please contact us if you need help. 
If you wish to gain access to WM and other SIMA Fault Tree Databanks you must fill out a CPC-16 form requesting an account to the SACDR1 node with read only access to the SIMA Fault Tree Databanks. The form must be signed by the level 3 manager and forwarded to SIMA' (K. A. Wellmaker, 992-W1) for additional approval. Upon receipt of the form by SIMA, training will be provided and a copy of the " Risk Assessment Methodology Fault Tree Databank User Guide (U)" , WSRC-IM-92-50, will be issued.

For more information on the SIMA databanks, please contact W. V. Minnick $992-$ $1 \mathrm{~W}$, ext. 4-5174, or K. A. Wellmaker, 992-1W, ext. 4-5087.

\section{AVAILABILITY}

WM Databank information is available to WSRC personnel upon request to the SIMA group. Printouts are usually available on the day of the request.

Distribution of the data outside of DOE or direct DOE contractors requires approval of DOE-SR. Direct access to the data for updates and changes is limited to authorized personnel to protect against unauthorized use and tampering. However, if errors are found, the customer is encouraged to contact SIMA so that corrections may be made.

Statistical analysis of data may be obtained from SIMA using a Work Authorization Document (WAD). The analysis includes both frequency and consequence determinations. Trend plots and statistical values such as mean, median, maximum, minimum, $90 \%$ bounds, sigma and error factor can be generated.

SIMA databank personnel are located in the Centennial Corporate Center, Building 992-1W
W. V. Minnick
Rm 349
644-5174
K. A. Wellmaker
Rm 238
644-5087
C. H. Sims
$\mathrm{Rm} 202$
664-5074
J.W. Abear
Rm 199 .
644-5039
S. B. Friedrichsen
Rm 201
644-5120
L. M. Adams
Rm 252
644-5406 


\section{TRAINING}

The Risk Assignment Methodology Fault Tree Databanks User's Guide (U), WSRC-IM-92-50 was developed for distribution to databank users. This manual is a self-help guide that starts with the sign-on screen and ends with the printed hard copy of your search. There are many options explained in the guide to help customers with their inquires. Training classes or individual training are available by contacting K. A. Wellmaker at extension 4-5087. If you intend to use the WM data for Technical Base Line Calculations you are required to note the admonishments shown on the computer screen and on each hard copy search, and advised to discuss the data limitations with SIMA personnel.

\section{SOURCES OF DATA}

As shown on table 3, data have been abstracted from 60 types of sources, 47 WSRC publications, and 6 unpublished sources such as log books. The range of dates for incidents abstracted from each of these sources is shown on data sheet 3.

Final data from published sources and one-year-old log books are coded on a continuous basis but an input lag of about a year is currently being experienced due to input resource limitations. Incidents as early as 1953 are included within the WM Databank. Potential data sources not listed in table 3 should be reported to K. A. Wellmaker at 644-5087.

\section{SEARCH OPTIONS}

Data may be searched by a variety of options as described in the user's guide. As an inquiry aid, each incident is assigned codes to identify: Area, Source of document [Table 3], Facility [Table 4], Operation [Table 5], Equipment Code or Key Word [Table 6, 7, and 9]. All search requests performed by the SIMA group for WSRC personnel will be mailed through the site mail or held at 992-1W for pickup.

\section{USAGE}

The WM Databank incidents have been applied to a wide range of applications.

- Some of these applications are shown in Table 10. 


\section{QUALITY ASSURANCE (QA)}

The SRS Fault Tree Databanks are categorized as a general service (GS) resource which provides a baseline for both QA protocol and user responsibility when utilizing output of the Databanks for Technical Baseline calculations (see sections 2.25, 2.31, and 2.32 of Conduct of Engineering and Technical Support (U), WSRC Procedure Manual E7). In general, raw data from the SRS Fault Tree Databanks cannot be used directly in critical calculations without proper qualification of data for that particular application. The proprietors of the Databanks (currently Safety Information Management Analysis In the Safety Engineering Department) will perform this function for customers upon request, or the user may choose to do so themselves.

Procedures have been developed that describe the key elements of the Databank support/maintenance. They document activities such as data extraction from sources, data entry, data editing, and sorts/searches. These procedures are intended to serve as guidelines for those performing the activities and to serve as resource for those involved in data qualification.

Efforts have also been concentrated in keeping users aware of Databank QA. In the opening screens of the data Bank user interface software, Databank users receive a written reminder of the QA categorization, including related obligations and responsibilities. In addition, new users are required to acknowledge receipt and familiarity with documentation regarding proper use of. Databank Information in critical applications.

\section{CODE UPGRADE PROGRAM}

The Waste Management Databank search codes have been revised to improve the user interface. The revision program required recoding 35,000 entries of the WM Databank. During the recoding task, published data were coded on a continuous basis and backlogged. These data are being entered; however, there will be a lag of approximately one year until the backlog can be worked off. 
TABLE 1

USAGE OF WM FACILITIES FAULT TREE DATABANK (Customer Requested Data Searches)

1994

E\&CSD

201

Safety Engineering Department

No. of

Year

Requesting Organization

Requests

1994

SRTC

3

Safety Technology

Site Configuration and Safety Services

199

2

1994

E\&PD

1994

SRS Site

Waste Management

Waste Management Technology

Waste Management Engineering

Tritium Engineering

Site Geo Technology

General Council Office.

Westinghouse-Hanford

SAIC

DOE-SR

Los Alamos National Laboratory

VECTRA

Radiological Assessment Corporation 


\section{TABLE 2}

AREA CODE DESIGNATION

\section{AREA CODES}

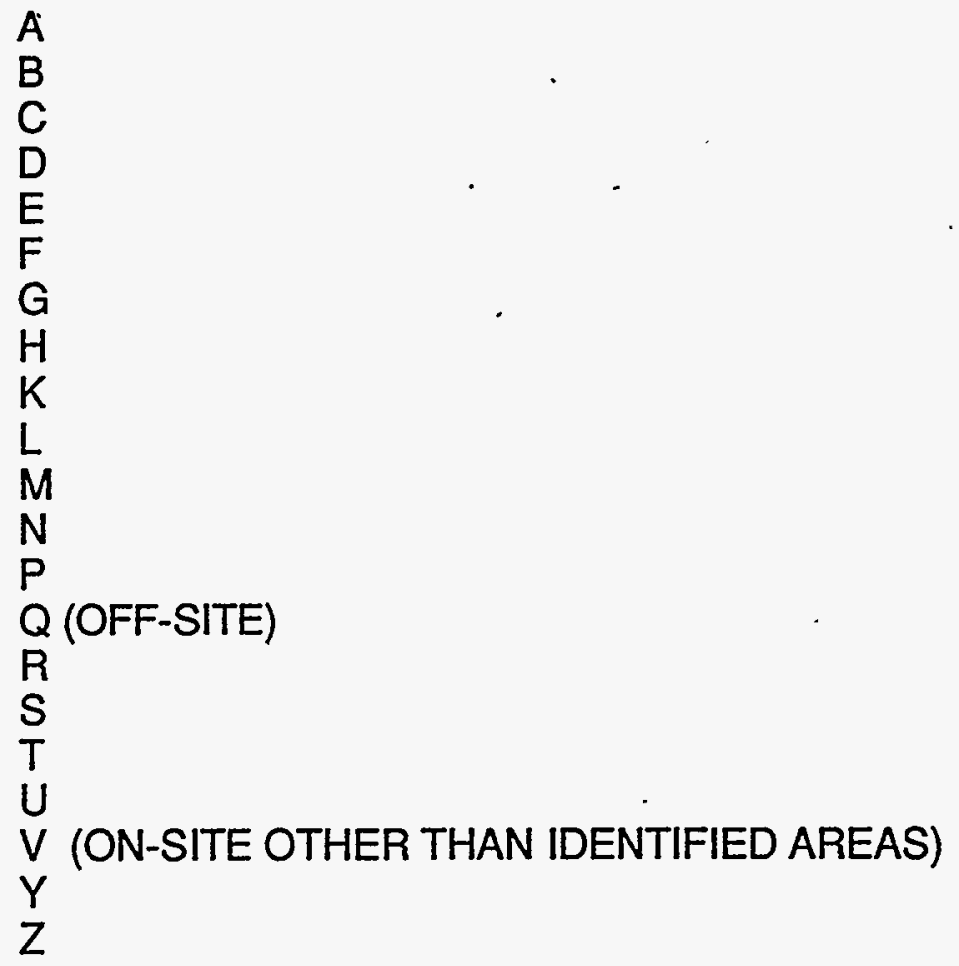


TABLE 3

SOURCE TITLE, SOURCE CODE, AND DATA RANGE

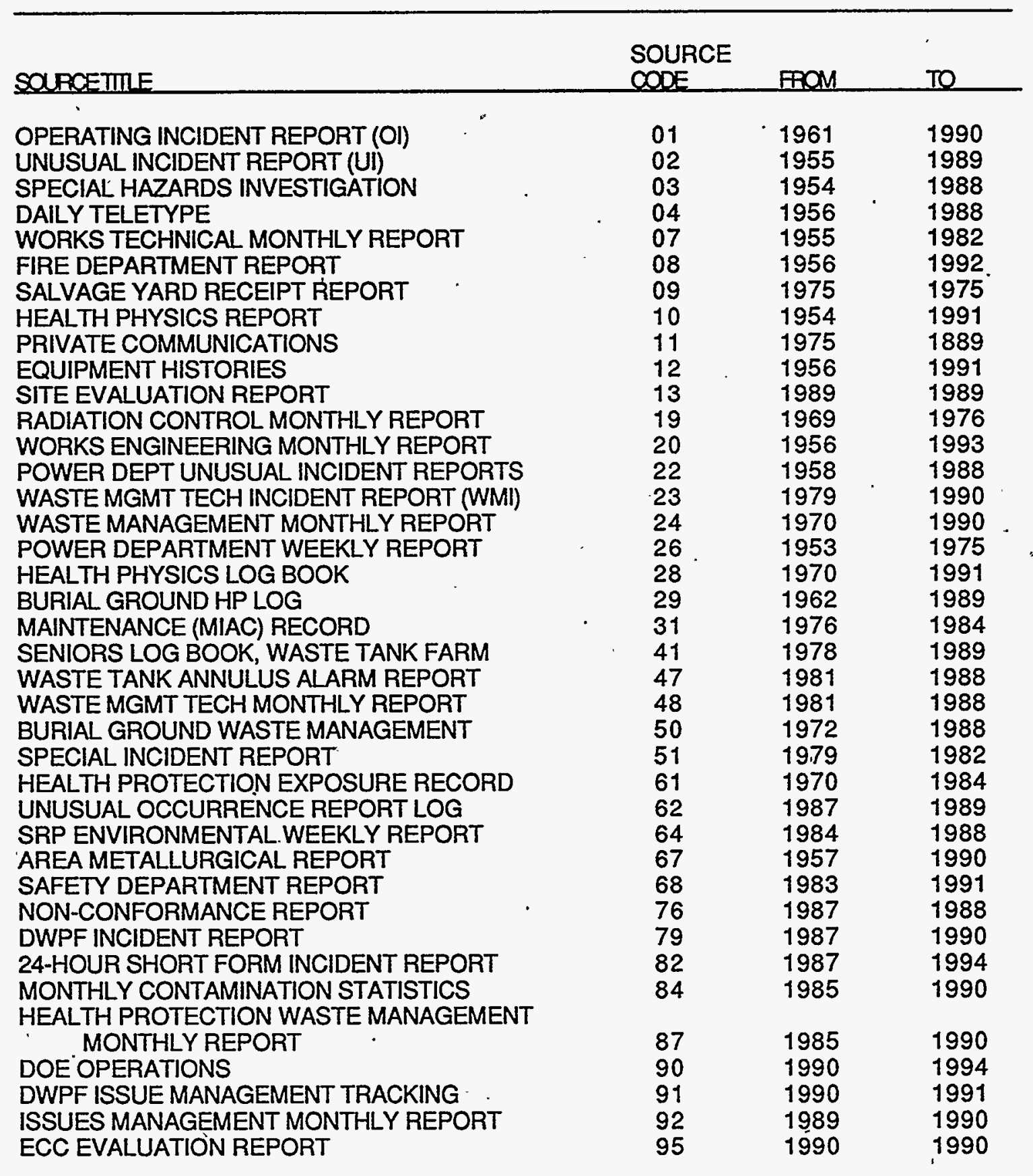


TABLE 3

SOURCE TITLE, SOURCE CODE, AND -DATA RANGE (Cont'd)

\begin{tabular}{|c|c|c|c|}
\hline SOURCETIIL & $\begin{array}{l}\text { SOURCE } \\
\text { CODE }\end{array}$ & FROM & TO \\
\hline $\begin{array}{l}\text { DWPF FINAL EVALUATION REPORT } \\
10 \text { DAY OCCURRENCE REPORT } \\
\text { FINAL OCCURRENCE REPORT } \\
\text { SAFETY NEWSLETTER } \\
\text { EMERGENCY OPERATIONS ACTIV. LOG (TSC) } \\
\text { SRTC SAFETY MORNING REPORT } \\
\text { SCRAP RECOVERY POWER SURVEILLANCE LOG } \\
\text { FIRE DEPARTMENT } 30 \text { DAY REPORT } \\
\text { HLWP (WM) DAILY OPERATIONS REPORT } \\
\text { SITE CENTRAL SAFETY COMM. MINUTES } \\
\text { SWM DAILY OPERATIONS REPORT } \\
\text { 24IF SUPERVISOR LOGSHEET } \\
\text { HIGH LEVEL WASTE DEPARTMENT OPERATIONS }\end{array}$ & $\begin{array}{l}97 \\
2 B \\
3 B \\
5 B \\
8 B \\
1 C \\
5 C \\
7 C \\
8 C \\
4 D \\
5 D \\
9 E\end{array}$ & $\begin{array}{l}1990 \\
1990 \\
1990 \\
1991 \\
1991 \\
1991 \\
1992 \\
1992 \\
1992 \\
1992 \\
1992 \\
1993\end{array}$ & $\begin{array}{l}1992 \\
1994 \\
1994 \\
1991 \\
1992 \\
1993 \\
1992 \\
1994 \\
1993 \\
1992 \\
1994 \\
1993\end{array}$ \\
\hline $\begin{array}{l}\text { MORNING REPORT } \\
\text { HIGH LEVEL WASTE DEPT WEEKLY REPORT } \\
\text { HIGH LEVEL WASTE DEPT DAILY REPORT } \\
\text { DWPF DAILY OPERATIONS REPORT } \\
\text { SRTC MATERIAL TECH SECTION MONTHLY REPORT } \\
\text { HIGH LEVEL WASTE ENGINEERING MONTHLY REPORT } \\
\text { LESSONS LEARNED NEWSLETTER } \\
\text { DOE OPERATING EXPERIENCE WEEKLY SUMMARY } \\
\text { THE 82-H CONTROL ROOM LOG } \\
\text { WASTE MANAGEMENT MORNING REPORT }\end{array}$ & $\begin{array}{l}1 G \\
2 G \\
3 G \\
4 G \\
5 G \\
6 G \\
7 G \\
8 G \\
3 C \\
9 B\end{array}$ & $\begin{array}{l}1993 \\
1993 \\
1993 \\
1993 \\
1993 \\
1993 \\
1994 \\
1994 \\
1989 \\
1991\end{array}$ & $\begin{array}{l}1994 \\
1994 \\
1994 \\
1994 \\
1994 \\
1994 \\
1994 \\
1994 \\
1992 \\
1991\end{array}$ \\
\hline
\end{tabular}


TABLE 4

FACILITY CODES

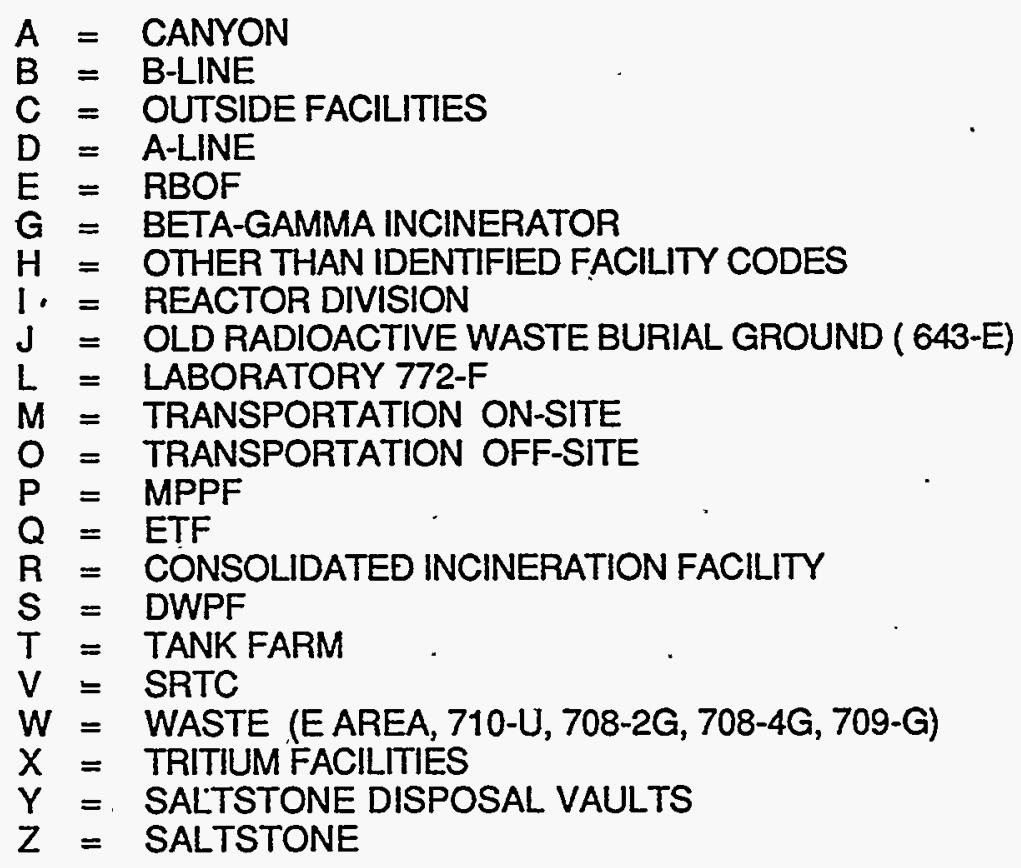


TABLE 5

OPERATION CODES (Cont'd)

Q3 QUALITY ASSURANCE

R1 RAILROAD TUNNELRAILROAD

R3 RESTORATION

S1 PERSONNEL SAFETY

S2 PROCESS SAFETY

S3 SALTSTONE

S5 SAMPLING

S7 SECURITY

S8 SHIPPING

T4 TANK (All references to tanks)

T5 TRANSFER (Inter Area, Intra Area Transfer Equipment, Pipe, Leak Detection and Diversion Boxes, 'etc.)

T7 TRAINING

U1 UTILITY (Water, Steam, Sanitary Sewer)

V1 VACUUM

V2 VEHICULAR

V5 VENTILATION

V7 VITRIFICATION

W1 WASTE-INTERMEDIATE LEVEL/HIGH LEVEL

W2 WASTE-HAZARDOUS

W3 WASTE-INTERIM STORAGE

W4 WASTE-SANITARY

W5 WASTE-TRENCH DISPOSAL

W6 WASTE-TRANSURANIC (TRU-Hazardous \& Non-hazardous)

W7 WASTE-VAULT DISPOSAL

W8 WASTE-MIXED

W9 WASTE-LOW LEVEL 


\section{TABLE 6}

EQUIPMENT CODES

(Alphabetical Order)

700

699

755

A

$\begin{array}{ll}094 & \text { ABSORBER } \\ 049 & \text { ABSORBER, ACID } \\ 683 & \text { ABSORPTION/UPTAKE } \\ 755 & \text { ACCEPTANCE CRITERIA } \\ 130 & \text { ACCOUNTABILITY } \\ 049 & \text { ACID, ABSORBER } \\ 963 & \text { ACID DRAIN CATCH TANK } \\ 786-901 & \text { ACID, FORMIC } \\ 044 & \text { ACID NITRIC } \\ 787 & \text { ACID OXALIC } \\ 037 & \text { ACTIVITY } \\ 226 & \text { ADDED, PROTECTIVE DEVICE } \\ 003 & \text { ADDITIONERROR, CHEMICAL } \\ 740 & \text { ADJUSTMENT TANK SLUDGE RECEIPT } \\ 149 & \text { ADJUSTMENT, VALENCE } \\ 822 & \text { ADMINISTRATIVE CONTROL, INADEQUATE } \\ 068 & \text { AGITATOR } \\ 988 & \text { AIR CONDITION } \\ 087 & \text { AIR LOCK } \\ 088 & \text { AIR REVERSAL } \\ 151 & \text { AIR, BREATHING } \\ 482 & \text { AIR, COMPRESSED } \\ 496 & \text { AIR, INSTRUMENT } \\ 705 & \text { AIR, PLANT } \\ 622 & \text { AIR, PROCESS } \\ 153 & \text { AIR BORNE CONTAMINATION } \\ 686 & \text { AIRCRAFT } \\ 989 & \text { ALGAE } \\ 282 & \text { ALARM } \\ 639 & \text { ALARM, FALSE } \\ 270 & \text { ALARM, HM } \\ 449 & \text { ALARM, TROUBLE } \\ 202 & \text { ALIGNMENT } \\ 133 & \text { ALKALI, CAUSTIC } \\ 554 & \text { AMERICIUM } \\ 526 & \text { AMERICIUM, CURIUM, CALIFORNIUM } \\ 502 & \text { AMMONIA COMPOUND } \\ 124 & \text { ANALYTICAL } \\ 754 & \text { ANALYZER, LOWER EXPLOSIVE LIMIT } \\ 882 & \text { ANALYZER, BENZENE } \\ 753 & \text { ANALYZER, HYDROGEN } \\ & \\ 0.9 & \end{array}$

10 GALLONS, GREATER THAN

10 GALLONS, LESS THAN

1006D. ACCEPTANCE CRITERIA ACID DRAIN CATCH TANK ACTIVITY ADJUSTMENT TANK SLUDGE RECEIPT ADJUSTMENT, VALENCE AGITATOR AIR LOCK AIR REVERSAL AIR, BREATHING AIR, COMPRESSED AIR, PLANT

AIR, PROCESS AIRCRAFT

ALGAE

ALARM

ALARM, HM

ALKALI, CAUSTIC AMMONIA COMPOUND ANALYTICAL ANALYZER, BENZENE ANALYZER, HYDROGEN 
TABLE 6

EQUIPMENT CODES (CONT'D)

(Alphabetical Order)

$\begin{array}{ll}902 & \text { ANALYZER, MOC } \\ 698 & \text { ANALYZER, OXYGEN } \\ 703 & \text { ANIMALINSECT/BIRD } \\ 436 & \text { ANNULUS } \\ 717 & \text { ANNULUS, INSPECTION } \\ 387 & \text { ANNULUS, VENTILATION } \\ 670 & \text { ARGON } \\ 702 & \text { ASH BUILDUP } \\ 486 & \text { ASPHALT/PAVEMENT } \\ 267 & \text { ASSEMBLY } \\ 942 & \text { AUTOMATIC TRANSFER SWITCH } \\ 802 & \text { AUX. PUMP PIT 512S }\end{array}$

B

B-25 CONTAINERS

B12 CONTAINERS

BAG .

666

BAG SEALER

BAG, DUST

BAIL, LIFTING

181

491

922

306

180

047

968

438

501

923

439

980

693

BALANCE

BARRICADE/FENCE/ENCLOSURE/DIKE

BASIN, DELAY

BASIN, RETENTION

BASIN, SEEPAGE

BASIN, STORAGE FUELTARGET

BATTERY

BEARINGS

BECHTEL

BELL

BELT

BETA GAMMA, INCINERATOR

BENZENE

BENZENE ANALYZER

882

582

BIOASSAY AND/OR CHEST COUNT

BIRD/ANIMALINSECT

BLANK

BLOW DOWN/UNPLUG/FLUSH

BLOW RING

BLOWER, FANS

BOARD/PANEL BOARD/CONTROL ROOM/CONTROL

BOILER

BOILOFF

BOILOVER

BOX, DIVERSION

BOX, LEAK DETECTION 
TABLE 6

EQUIPMENT CODES (CONT'D)

(Alphabetical Order)

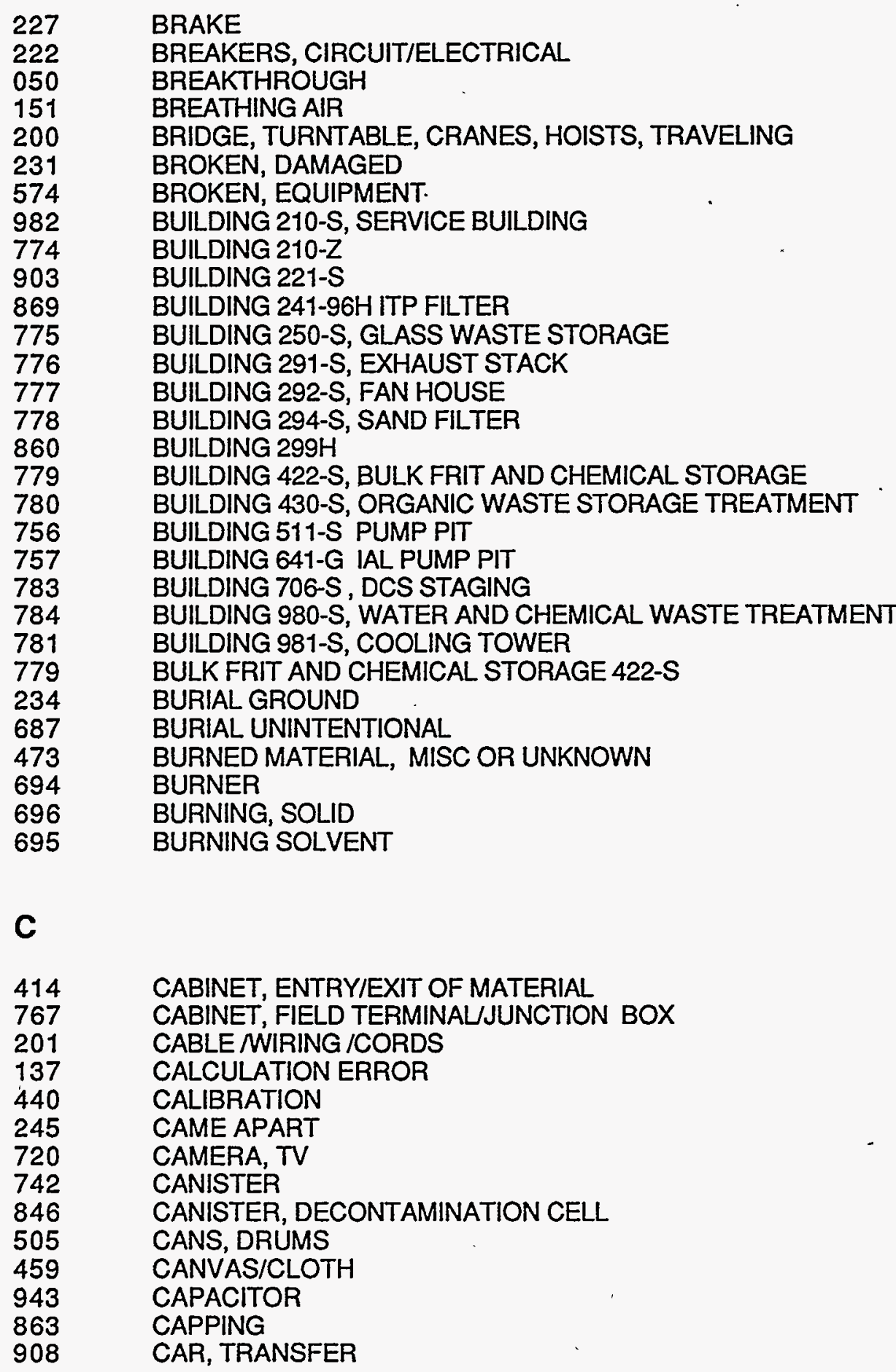


TABLE 6

EQUIPMENT CODES (CONT'D)

(Alphabetical Order)

$\begin{array}{ll}524 & \text { CAR, TRUCK } \\ 210 & \text { CAR/RAIL CAR, CASK } \\ 871 & \text { CARD/MODULE/BOARD } \\ 684 & \text { CART } \\ 628 & \text { CASH COIL PRESSURE SYSTEM } \\ 207 & \text { CASK } \\ 210 & \text { CASK, CAR/RAIL CAR } \\ 188 & \text { CATALYST } \\ 979 & \text { CATCH TANK } \\ 963 & \text { CATCH TANK, ACID DRAIN } \\ 962 & \text { CATCH TANK, FLOOR DRAIN } \\ 133 & \text { CAUSTIC ALKALI } \\ 846 & \text { CELL, CANISTER DECONTAMINATION } \\ 172 & \text { CELL COVER } \\ 844 & \text { CELL, CHEMICAL PROCESSING } \\ 182 & \text { CELL, DECONTAMINATION } \\ 847 & \text { CELL, MAINTENANCEIDECONTAMINATION } \\ 850 & \text { CELL, MELTER } \\ 851 & \text { CELL, PROCESS } \\ 845 & \text { CELL, SALT PROCESS } \\ 849 & \text { CELL, SAMPLE AND ANALYTICAL } \\ 277 & \text { CELL, WASTE } \\ 848 & \text { CELL, WELD } \\ 768 & \text { CENTER, LOAD } \\ 625 & \text { CENTER, MOTOR CONTROL } \\ 441 & \text { CHAMBER } \\ 429 & \text { CHANGE, GLOVE } \\ 492 & \text { CHARGER/GENERATOR } \\ 433 & \text { CHECK, LEAK } \\ 824 & \text { CHECK, SOURCE } \\ 003 & \text { CHEMICAL ADDITION ERROR } \\ 690 & \text { CHEMICALS, HAZARDOUS } \\ 836 & \text { CHEMICAL/NDUSTRIAL WASTE TREATMENT } \\ 779 & \text { CHEMICAL STORAGE BLDG. 422-S, BULK FRIT } \\ 784 & \text { CHEMICAL WASTE TREATMENT BLDG. 980-S WATER \& } \\ 582 & \text { CHEST COUNT AND/OR BIOASSAY } \\ 764-935 & \text { CHILLERS PROCESS } \\ 821 & \text { PROCESS, WATER } \\ 065 & \text { CHIPPED/CRACKS/CRACKING } \\ 452 & \text { CHLORIDES } \\ 513 & \text { CHOCKS } \\ 870 & \text { CHROMATED WATER } \\ 966 & \text { CIRCUIT } \\ 218 & \text { CIRCUIT, ELECTRICAL SHORT } \\ 222 & \text { CIRCUIT/ELECTRICAL BREAKERS } \\ 950 & \text { CLAYISOILS } \\ 260 & \text { CLOSED VESSEL ENTRY } \\ 512 & \text { CLOSURE, TIE DOWN } \\ & \\ & \end{array}$


TABLE 6

EQUIPMENT CODES (CONT'D)

(Alphabetical Order)

$\begin{array}{ll}459 & \text { CLOTH/CANVAS } \\ 672 & \text { CLOTHING, CONTAMINATION } \\ 877 & \text { CLOTHING, PERSONALIEFFECTS } \\ 079 & \text { CLOTHING, PROTECTIVE } \\ 401 & \text { COAL } \\ 494 & \text { COATINGILINER } \\ 014 & \text { COIL LEAK/TUBE BUNDLE LEAK } \\ 628 & \text { COIL, CASH PRESSURE SYSTEM } \\ 167 & \text { COIL, COOLING } \\ 132 & \text { COIL, HEATING } \\ 825 & \text { COLD FEED PREP } \\ 766 & \text { COLLAPSE STEAM } \\ 080 & \text { COLLECTION, DUST } \\ 770 & \text { COLLECTION TANK, RECYCLE } \\ 504 & \text { COLLISION } \\ 886 & \text { COLUMN, CESIUM REMOVAL } \\ 043 & \text { COLUMN FLOODING } \\ 743 & \text { COLUMN, MERCURY PURIFICATION } \\ 119 & \text { COLUMNS, DEIONIZERIION EXCHANGE } \\ 795 & \text { COLUMN, STRIPPER } \\ 868 & \text { COMMUNICATION, INADEQUATE } \\ 159 & \text { COMMUNICATION, PHONE } \\ 806 & \text { COMMUNICATION SYSTEM } \\ 697 & \text { COMBUSTION, PROGRAMMERS } \\ 453 & \text { COMBUSTION, SPONTANEOUS } \\ 726 & \text { COMPACTOR, WASTE } \\ 225 & \text { COMPONENT, FAULTY } \\ 217 & \text { COMPONENT, JAMMED } \\ 233 & \text { COMPONENT STRUCTURAL } \\ 502 & \text { COMPOUNDS, AMMONIA } \\ 482 & \text { COMPRESSED AIR } \\ 110 & \text { COMPRESSOR } \\ 481 & \text { COMPUTER } \\ 803 & \text { COMPUTER PROGRAM ERROR } \\ 712 & \text { CONCENTRATE, TRANSFER SYSTEM } \\ 633 & \text { CONCRETE } \\ 855 & \text { CONDENSATE, TANK } \\ 034 & \text { CONDENSER } \\ 880 & \text { CONDUCT OF OPERATIONS } \\ 929 & \text { CONNECTOR } \\ 558 & \text { CONSTRUCTION DIVISION } \\ 224 & \text { CONTACT, ELECTRICAL } \\ 724 & \text { CONTAINER, UNAUTHORIZED } \\ 239 & \text { CONTAINER, SHIPPING } \\ 143 & \text { CONTAINERNESSELITANK } \\ 746 & \text { CONTAINERS, B-25 } \\ 751 & \text { CONTAMINATION } \\ 153 & \text { CONTAMINATION, AIRBORNE } \\ & \\ & \end{array}$


TABLE 6

EQUIPMENT CODES (CONT'D)

(Alphabetical Order)

$\begin{array}{ll}672 & \text { CONTAMINATION, CLOTHING } \\ 048 & \text { CONTAMINATION, FACILITY OR EQUIPMENT } \\ 875 & \text { CONTAMINATION, FIXED } \\ 682 & \text { CONTAMINATION, NASAL } \\ 548 & \text { CONTAMINATION, SKIN } \\ 877 & \text { CONTAMINATION, PERSONAL CLOTHING/EFFECTS } \\ 804 & \text { CONTAMINATION, PERSONNEL MONITOR } \\ 811 & \text { CONTAMINATION, PROCESS MONITOR } \\ 876 & \text { CONTAMINATION, SMEARABLE } \\ 096 & \text { CONTROL, FLOW } \\ 822 & \text { CONTROL, INADEQUATE ADMINISTRATIVE } \\ 680 & \text { CONTROLLER } \\ 625 & \text { CONTROL, MOTOR CENTER } \\ 759 & \text { CONTROL, PRESSURE } \\ 873 & \text { CONTROL, PROGRAMMABLE LOGIC } \\ 179 & \text { CONTROL ROOM/CONTROL BOARD/PANEL BOARD } \\ 723 & \text { CONTROL, QUALITY } \\ 899 & \text { CONTROL SYSTEM, DISTRIBUTIVE } \\ 062 & \text { CONTROL, TEMPERATURE } \\ 790 & \text { CONVEYOR } \\ 801 & \text { CONVEYOR, PNEUMATIC } \\ 086 & \text { CONVEYOR, SCREW } \\ 167 & \text { COOLING COIL } \\ 305 & \text { COOLING TOWER } \\ 781 & \text { COOLING TOWER, BUILDING 981-S } \\ 045 & \text { COOLING WATER } \\ 201 & \text { CORD/CABLEMIRING } \\ 016 & \text { CORROSION } \\ 749 & \text { CORROSION (MIC) MICRO BIOLOGICALLY INDUCED } \\ 985 & \text { COUNTER/TIMER/INTEGRATOR } \\ 172 & \text { COVER, CELL } \\ 065 & \text { CRACKS/CRACKING/CHIPPED } \\ 200 & \text { CRANES/HOISTSTTRAVELING BRIDGETTURN TABLE } \\ 807 & \text { CRANE, INCELL } \\ 046 & \text { CREEK, FOUR MILE } \\ 095 & \text { CREEK, THREE RUNS } \\ 755 & \text { CRITERIA, ACCEPTANCE } \\ 005 & \text { CRITICALITYPOTENTIAL } \\ 522 & \text { CROSSING, RR } \\ 117 & \text { CRUCIBLE } \\ 719 & \text { CTS } \\ 948 & \text { CTS LOOP } \\ 738 & \text { CUTTER, PIPE } \\ 291 & \text { CUTTING } \\ & \end{array}$




\section{TABLE 6}

EQUIPMENT CODES (CONT'D)

(Alphabetical Order)

D

D20/TRITIUM

DAMAGE, MECHANICAL

DAMAGE, ELECTRICAL EQUIPMENT

DAMAGED/BROKEN

DAMAGED, TRANSPORTATION EQUIPMENT

DAMPER

DATE, OUT OF

DCS STAGING BLDG. 706-S

DEBRIS

DECANT

DECAYIDECOMPOSITION

DECANTER

DECAY HEAT

DECOMMISSION/DEMOLITION

DECOMPOSITION/DECAY

DECONTAMINATION CELL. .

DECONTAMINATION

DEFICIENCY, WORK ORDER

DEFLAGRATION

DEFORMATION

DEHUMIDIFIER

DEIONIZER/ION EXCHANGE COLUMN

DELAY BASIN

DEMISTER

DEMOLITION/DECOMMISSION

DEPARTMENT, REACTOR.

DESIGN

DESIGN, ERROR/IMPROVEMENT

DESALTIDESCALE

DETECTION, FIRE AND SUPPRESSION

DETECTOR

DEVICE ADDED, PROTECTIVE

DEVICE MISSING, PROTECTIVE

DEVICE, TRANSFER

DEWATERING FACILITY

DIAPHRAGM

DIES

DIESEL

DIFFICULTY, PERSONNEL

DIFFICULTY, PROCEDURAL

DIGGING

DIKE/FENCE/BARRICADE/ENCLOSURE

DIMENSIONS

DIODE

DIP TUBE

DISK, RUPTURE 
TABLE 6

EQUIPMENT CODES (CONT'D)

(Alphabetical Order)

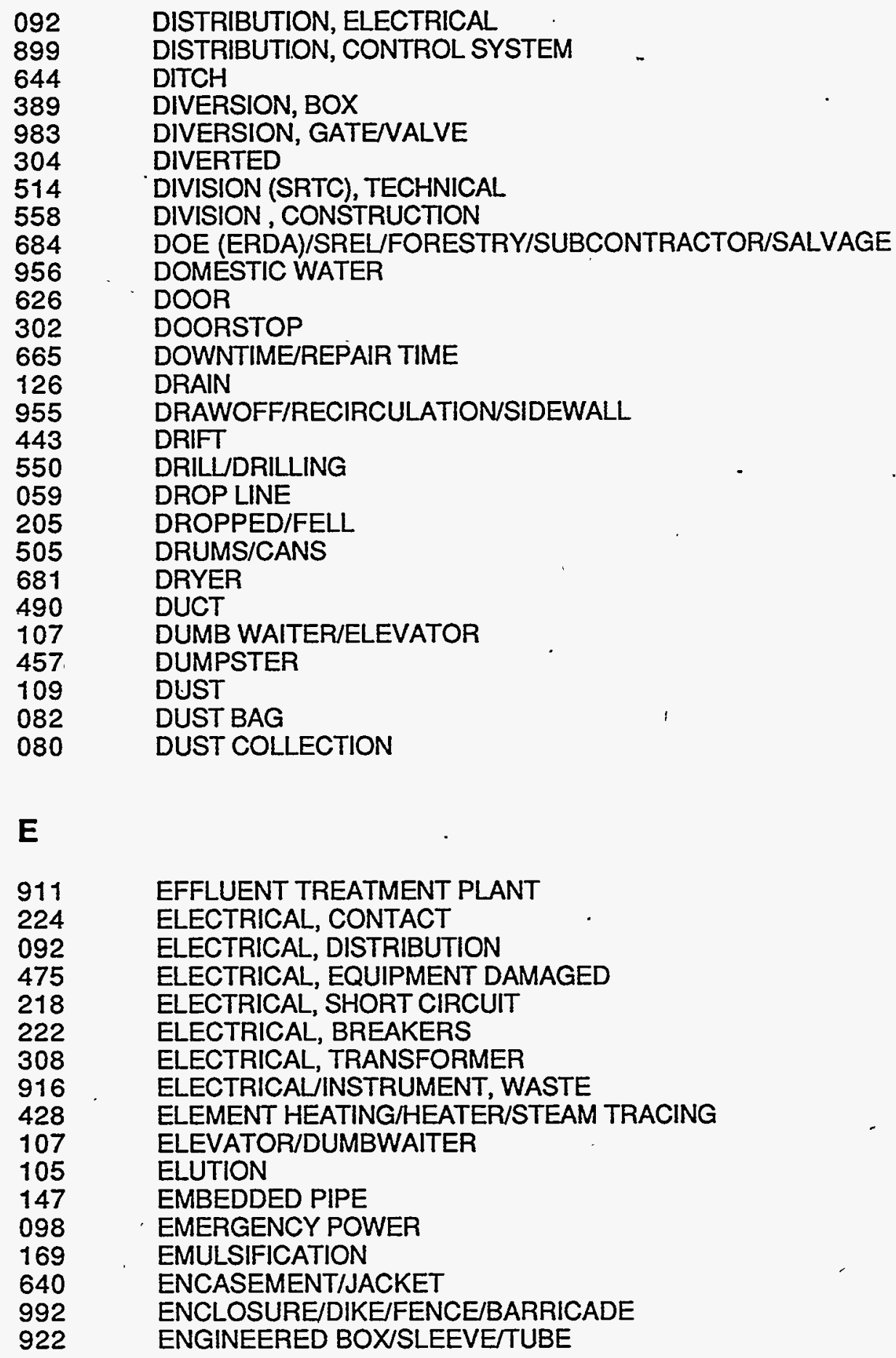


TABLE 6

EQUIPMENT CODES (CONT'D)

(Alphabetical Order)

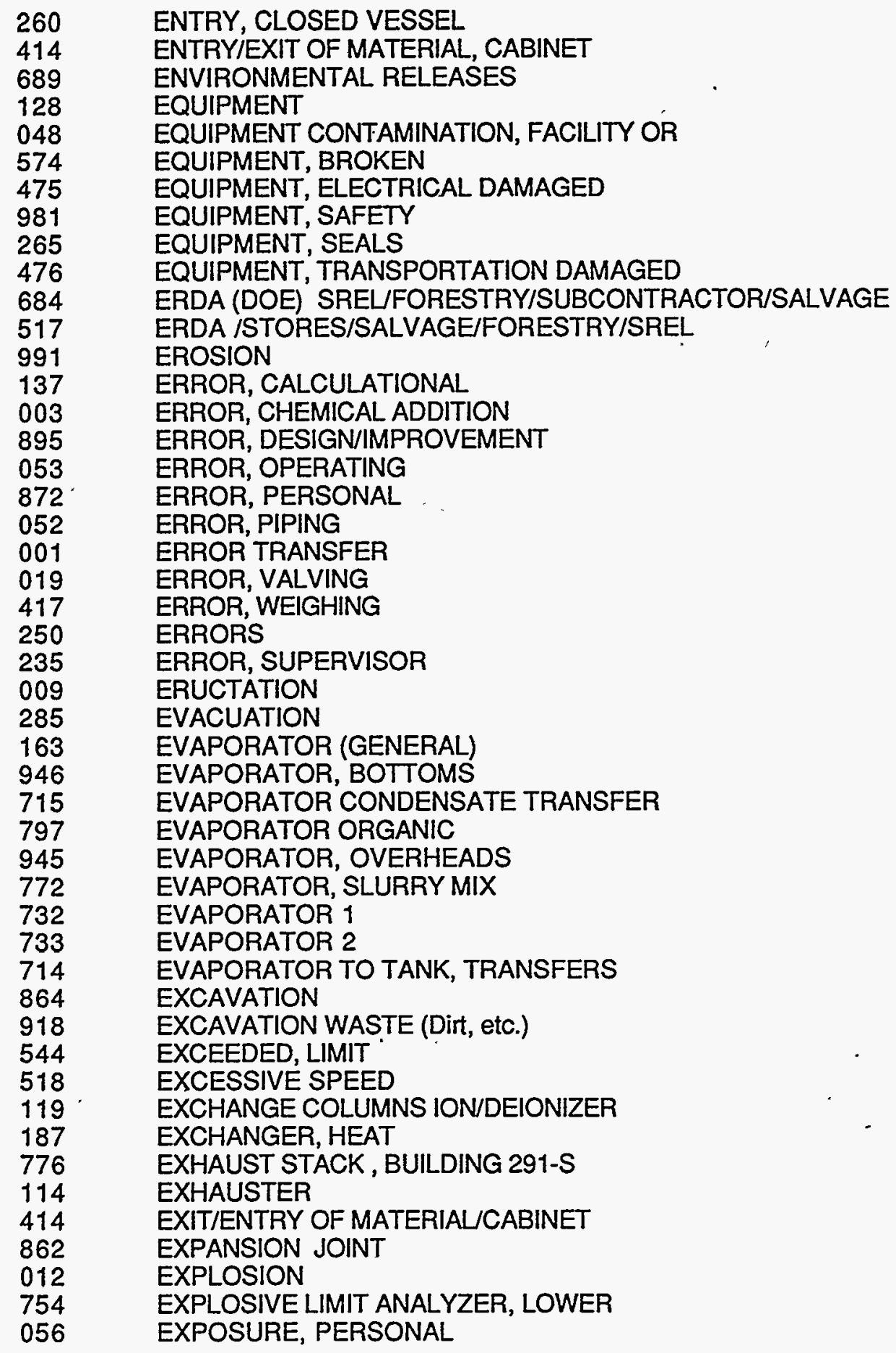


TABLE 6

EQUIPMENT CODES (CONT'D)

(Alphabetical Order)

$\mathbf{F}$

FACILITY BLDG. 430-S, ORGANIC WASTE STORAGE

FACILITY OR EQUIPMENT CONTAMINATION

FACILITY, DEWATERING

FACILITY, LATE WASH

FACILITY, SOLID WASTE DISPOSAL

FAILURE, MECHANICAL

FAILURE, VALVE

FALSE

FALSE ALARM

FAN HOUSE, BUILDING 292-S

FANS / BLOWER

FATALITIES

FAULTY COMPONENT

FAULTY/MALFUNCTION

FEED PUMP

FEED TANK

FENCE/BARRICADE/ENCLOSURE/DIKE

FELLIDROPPED

FIELD TERMINAL CABINET/JUNCTION BOX

FILL, SANITARY LAND FILL

OIL FILM

FILTER, BUILDING 294-S, SAND

FILTER, HEPA

FILTER, SAND

FILTER (GENERAL) / SCREENS/STRAINERS

FIRE

FIRE DETECTION EQUIPMENT

FITTINGS

FIXED CONTAMINATION

FLANGE/GASKET

FLASHER

FLOOD/DIVERSION/SLUICE, GATENALVE

FLOODED

FLOODING, COLUMN

FLOOR

FLOOR, HIT

FLOOR, HIT OTHER THAN

FLOW

FLOW CONTROL

FLOWMETER/ROTAMETER

FLUID, HYDRAULIC

FLUORINE COMPOUNDS

949

FLUSH / BLOWDOWN / UNPLUG 
TABLE 6

EQUIPMENT CODES (CONT'D)

(Alphabetical Order).

645

021

684

517

808

786-901

960

529

046

466

798

779

042

230

069

221

676

102

425

422

761

G

287 GAGE, INSTRUMENT

983

157

920

472

101

024

468

455

455

051

492

131

818

741

262

775

429

111

463

700

407

295

FOAMING

FORMATION, SALT

FORMIC ACID

FOUR MILE CREEK

FRICTION

FRIT

FROZEN, ICE

FUEL

FUMES

FUNNEL/HOPPER

FURNACE/OVEN

FURNACE, VACUUM

FUSE

GANG VALVE

GAS

GAS, INERT

GASKET/FLANGE

GASOLINE

GEAR BOXJGEARS

GEARS/GEAR BOX

GEL, SILICA

GLASS

GLASS MELTER

GLASS, SIGHT

GLOVE CHANGE

GLOVES

GRASS

GROUND WATER

\section{FLUSH WATER}

FORESTRY / SREL / DOE (ERDA)/ SUBCONTRACTOR / SALVAGE

FORESTRY/STORES/SALVAGE/SREL/ERDA

FORMIC ACID WASTE HEADER

FORK LIFT/MOTOR CRANE/TRACTOR

FRIT \& CHEMICAL STORAGE, BLDG. 422-S, BULK

FUNCTION, INADEQUATE

FURNACE, INDUCTION

GATE, DIVERSION/FLOOD/SLUICE

GANG VALVE HOUSE

GENERATOR/CHARGER

GENERATOR, MOTOR

GLASS WASTE STORAGE BUILDING, 250-S

GREATER THAN 10 GALLONS

GRINDINGMACHINING 
TABLE 6

EQUIPMENT CODES (CONT'D)

(Alphabetical Order)

234 GROUND, BURIAL

619 GUIDE, RELEASE

H

673 HALON

403 WATER HAMMER

171 HAN

209 HAND TOOLS

690 HAZARDOUS CHEMICALS

927 HAZARDOUS METALS

840 HAZARDOUS/MIXED WASTE VAULT

832 HAZARDOUS WASTE, INTERIM STORAGE

909 HAZARDOUS WASTE

136 HEADER

960 HEADER, FORMIC ACID WASTE

$958^{\circ} \quad$ HEADER, HOT DECON WASTE

961 HEADER, MERCURY TRANSFER

959 HEADER, WARM DECON WASTE

187 HEAT EXCHANGER/COOLER

170 HEAT, DECAY

456 HEAT, PROCESS

728

428

132

668

638

685

214

215

270

200

213

676

261

777

145

936 .

150

692

118

198

753

HEAT $/ T E M P E R A T U R E$

HEATER/HEATING ELEMENT/STEAM TRACING

HEATING COILNAPORIZER

HELIUM

HEPA FILTER

HIGHWAY 125

HIT FLOOR

HIT OTHER THAN FLOOR

HM ALARM

HOISTS/TRAVELING BRIDGEITURNTABLES/CRANES

HOOK

HOPPER/FUNNEL

HOSE

HOUSE, BUILDING 292-S, FAN

HUMIDITY

HUMAN FACTÓRS

HUT

HYDRAULIC FLUID

HYDRAULIC SYSTEMS

HYDROGEN

HYDROGEN ANALYZER

889

HYDROTEST 
TABLE 6

EQUIPMENT CODES (CONT'D)

(Alphabetical Order)

I

042

ICE, FROZEN

244

IDENTIFICATION, FUEL

IGNITION SOURCE, MISC. OR UNKNOWN

978

249

725

125

543

868

221

506

395

535

807

693

235

237

425

836

937

101

155

156

478

479

077

216

703

238

718

551

717

IMPACT WRENCH

IMPELLER

IMPROPER PACKAGING

IMPROPER STORAGE

IMPURITIES

INADEQUATE

INADEQUATE COMMUNICATION

INADEQUATE FUNCTION

INADEQUATE MONITORING

INADEQUATE, PROCEDURE

INATTENTION

INCELL, CRANE

INCINERATOR, BETA-GAMMA

INCOMPLETE JOB

INCORRECT POSITION

INDUCTION, FURNACE

INDUSTRIAL/CHEMICAL WASTE TREATMENT

INDUSTRIAL HYGIENE

INERT GAS

INGESTION/UPTAKE

INHALATION

INHALATION/UPTAKE

INJECTION/UPTAKE

INJURY

INJURY, POTENTIAL MAJOR

INSECT/ANIMAL/BIRD

INSECURE

INSIDE TANK, INSPECTION

INSPECTION

INSPECTION, ANNULUS

INSPECTION, INSIDE TANK

INSTALL/REPLACE/REMOVE

INSTRUMENT AIR

INSTRUMENT MALFUNCTION

INSTRUMENT, GAGE

INSTRUMENT, METEOROLOGICAL

INSTRUMENT/EQUIPMENT, WASTE ELECTRICAL

INSULATION/INSULATOR

462

767

545

985

834

INTER AREA HWE PUMP PIT, BLDG 641-G

INTERLOCK

INTEGRATOR/COUNTER/TIMER

INTERMEDIATE LEVEL WASTE

839

INTERMEDIATE LEVEL WASTE,

NON TRITIUM VAULT 
TABLE 6

EQUIPMENT CODES (CONT'D)

(Alphabetical Order)

$\begin{array}{ll}289 & \text { INVENTORY } \\ 194 & \text { IODINE. } \\ 119 & \text { ION EXCHANGE COLUMN/DEIONIZER } \\ 716 & \text { IRREGULARITIES, PROCESS. } \\ & \\ \mathbf{J} & \\ & \\ 530 & \text { JACK KNIFE } \\ 640 & \text { JACKET/ENCASEMENT } \\ 116 & \text { JACKET LEAK } \\ 217 & \text { JAMMED COMPONENT } \\ 017 & \text { JET } \\ 236 & \text { JOB, INCOMPLETE } \\ 862 & \text { JOINT, EXPANSION } \\ 917 & \text { JOB CONTROL WASTE } \\ 236 & \text { JOB, INCOMPLETE } \\ 767 & \text { JUNCTION.BOXIFIELD TERMINAL CABINET } \\ 025 & \text { JUMPER } \\ & \\ K & \end{array}$

$\mathbf{L}$

$\begin{array}{ll}986 & \text { LATE WASH FACILITY } \\ 691 & \text { LAZY SUSAN } \\ 433 & \text { LEAK CHECK } \\ 014 & \text { LEAK, COILTUBE BUNDLE } \\ 888 & \text { LEAK, PIPE } \\ 931 & \text { LEAK DETECTION, SUMP/BOX/POTS } \\ 008 & \text { LEAKS } \\ 699 & \text { LESS THAN 10 GALLONS } \\ 500 & \text { LEVEL, LIQUID } \\ 181 & \text { LIFTING, BAIL } \\ 072 & \text { LIGHTNING } \\ 263 & \text { LIGHTS } \\ 754 & \text { LIMIT ANALYZER, LOWER EXPLOSIVE } \\ 544 & \text { LIMIT EXCEEDED } \\ 228 & \text { LIMIT SWITCH } \\ 142 & \text { LIMITS, TECHNICAL STANDARDS/OPERATING } \\ 059 & \text { LINE, DROP } \\ 494 & \text { LINER/LINING (COATING) } \\ 500 & \text { LIQUID, LEVEL } \\ 768 & \text { LOAD CENTER }\end{array}$


TABLE 6

EQUIPMENT CODES (CONT'D)

(Alphabetical Order)

LOAD, LOSS OF

087

809

259

418

519

192

525

258

773

994

LOCK, AIR

LOCK AND TAG VIOLATION

LOCKED OUT

LOCKS TRANSFER

LOCOMOTIVE

LOSS

LOSS OF LOAD

LOSS OF MATERIAL

LOW POINT PUMP PIT 511-S

LYSIMETER

M

407 MACHINING/GRINDING

706

847

454

900

763

541

216

MAGNET

MAINTENANCE/DECONTAMINATION CELL

MAINTENANCE OPERATION

MAINTENANCE PREDICTIVE

MAINTENANCE PREVENTATIVE

MAINTENANCE ROOM,

MAJOR INJURY

MALFUNCTION/NSTRUMENT

MALFUNCTION, FAULTY

MANIPULATOR

MASS SPECTROMETER

MATERIAL BURNED, MISC. OR UNKNOWN

MATERIAL LOST

MATERIAL, CABINET ENTRY/EXIT OF

MATERIAL, SMOKING

MATERIAL, UNKNOWN

MECHANICAL DAMAGE

MECHANICAL FAILURE

MELTER CELL

MELTER , GLASS

MERCURY

MERCURY PURIFICATION COLUMN

MERCURY TRANSFER HEADER

METAL

METAL, PROCESS SCRAP

METALS, HAZARDOUS (CD, PB, HG, AG, etc.)

METEOROLOGICAL INSTRUMENTS

METER

MICRO BIOLOGICALLY INDUCED CORROSION (MIC)

MILE CREEK, FOUR

MISC. OR UNKNOWN IGNITION SOURCE

473

MISC. OR UNKNOWN MATERIAL BURNED 
TABLE 6

EQUIPMENT CODES (CONT'D)

(Alphabetical Order)

\section{MISLABELED}

MISSILES

MISSING/UNAVAILABLE

MISSING, PROTECTIVE DEVICE

MIX EVAPORATOR, SLURRY

MIXED/HAZARDOUS WASTE VAULT

MIXED WASTE.TREATMENT AND STORAGE, INTERIM

MIXING, SALT/CEMENT

737

804

805

450

279

MONITOR, PERSONNEL CONTAMINATION

MONITOR, PERSONNEL RADIATION

MONITOR, NEUTRON

MONITOR, STACK

636

549

506

208

066

625

529

131

MONITOR, TREBLER

MONITORING

MONITORING, INADEQUATE

MONORAIL

MOTOR

MOTOR CONTROL CENTER

MOTOR CRANE/FORK LIFT/TRACTOR

MOTOR GENERATOR

$\mathbf{N}$

682 NASAL, CONTAMINATION

041

104

450

097

898

044

897

758

084

271

168

NATURAL PHENOMENON

NEPTUNIUM

NEUTRON MONITOR

NIM

NITRATE, SODIUM

NITRIC ACID

NITRITE, SODIUM

NITROGEN

NOOTER

NOT LOCKED OUT OR TAGGED OUT

NOZZLE

0

$\begin{array}{ll}071 & \text { OFF-GAS } \\ 186 & \text { OFF-SITE } \\ 929 & \text { OIL } \\ 281 & \text { OIL FILM } \\ 053 & \text { OPERATING ERROR } \\ 142 & \text { OPERATING LIMITSITECHNICAL STANDARDS } \\ 880 & \text { OPERATIONS, CONDUCT OF } \\ 454 & \text { OPERATION, MAINTENANCE }\end{array}$


TABLE 6

EQUIPMENT CODES (CONT'D)

(Alphabetical Order)

P

OPERATIONAL SAFETY REQUIREMENT / TECHNICAL SAFETY REQUIREMENT OPTICS ORGANIC

ORGANIC EVAPORATOR ORGANIC, WASTE STORAGE

ORGANIC, WASTE STORAGE FACILITY BUILDING 430-S OSMOSIS, REVERSE OSR / TSR VIOLATION

OUT, LOCKED

OUT OF DATE

OUTAGE, POWER

OUTLET/WEIR

OVEN / FURNACE

OVERCONCENTRATION

OVERFLOW

OVERLOAD

OVERTURN

OXALIC ACID

OXYGEN ANALYZER

OXYGEN ANALYZER MOC

PACKAGING, IMPROPER

PACKAGING, PRIMARY

PACKAGING SECONDARY

PACKING / SEALS / CAULKING .

PANEL BOARD / CONTROL ROOM / CONTROL BOARD

PAPER

PAPERS, SHIPPING

PAVEMENT

PERMIT VIOLATION

PERSONAL CLOTHING

PERSONAL ERROR

PERSONNEL

PERSONNEL CONTAMINATION MONITOR

PERSONNEL DIFFICULTY

PERSONNEL RADIATION MONITOR

PHA

PHENOMENON, NATURAL

PHONE, COMMUNICATION

PIPE

PIPE CUTTER

PIPE, EMBEDDED

PIPE LEAK

PIPE, RACK

PIPING, ERROR 
TABLE 6

EQUIPMENT CODES (CONT'D)

(Alphabetical Order)

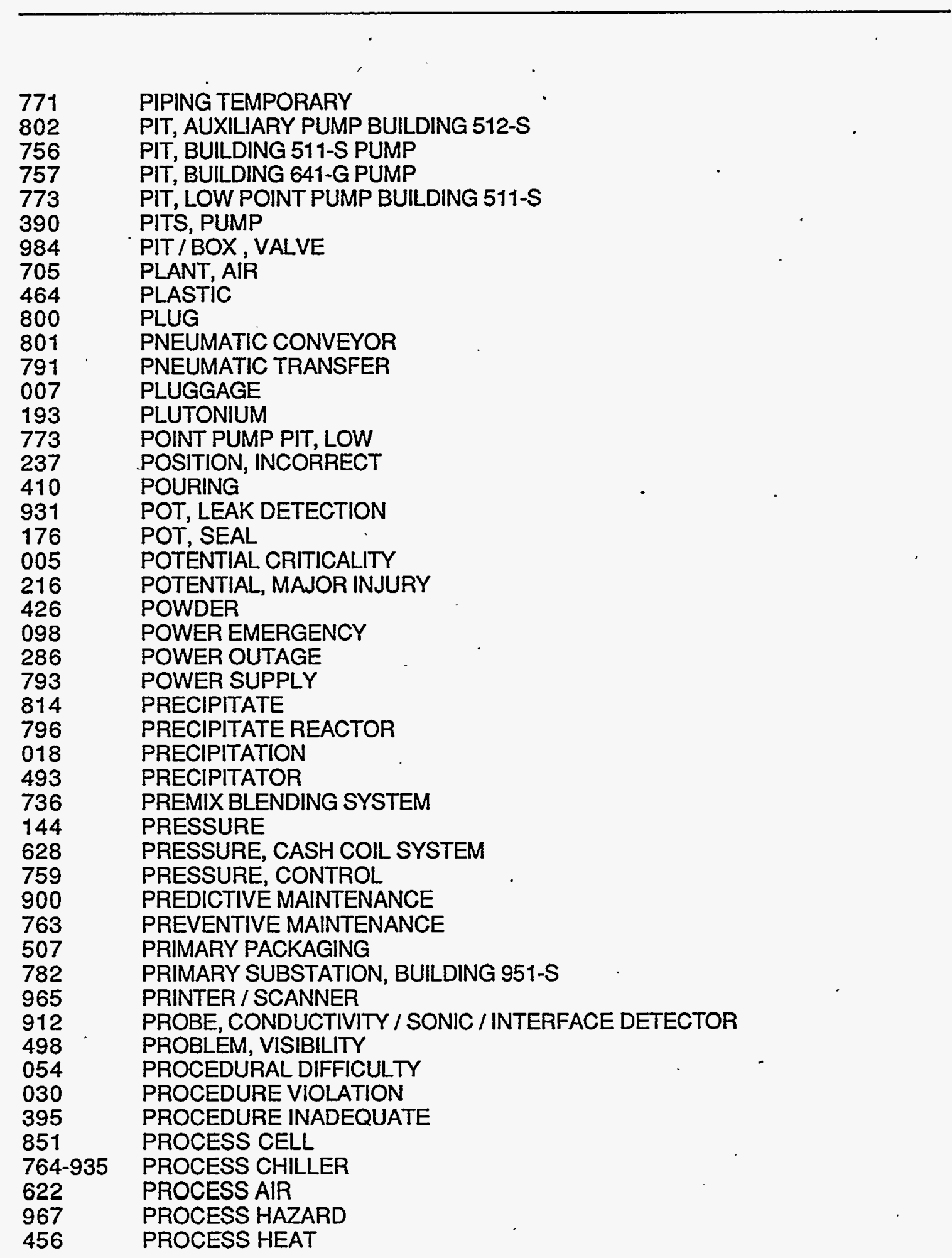




\section{TABLE 6}

EQUIPMENT CODES (CONT'D)

(Alphabetical Order)

$\begin{array}{ll}716 & \text { PROCESS IRREGULARITIES } \\ 036 & \text { PROCESS WATER } \\ 810 & \text { PROCESS CONTAMINATION MONITOR } \\ 811 & \text { PROCESS RADIATION MONITOR } \\ 697 & \text { PROGRAMMERS, COMBUSTION } \\ 079 & \text { PROTECTIVE CLOTHING } \\ 226 & \text { PROTECTIVE DEVICE ADDED } \\ 229 & \text { PROTECTIVE DEVICE MISSING } \\ 533 & \text { PULPWOOD TRUCK } \\ 085 & \text { PULVERIZER } \\ 091 & \text { PUMP } \\ 933 & \text { PUMP, FEED } \\ 906 & \text { PUMP HOUSE } \\ 756 & \text { PUMP PIT BUILDING 511-S } \\ 390 & \text { PUMP, PITS } \\ 265 & \text { PUMP SEALS } \\ 815 & \text { PUMP, SLURRY } \\ 892 & \text { PUMP, TANK } \\ 934 & \text { PUMP, TRANSFER } \\ 141 & \text { PUNCTURE } \\ 100 & \text { PURGING } \\ 743 & \text { PURIFICATION, MERCURY COLUMN }\end{array}$

$\mathbf{Q}$

723 QUALITY CONTROL

701 QUENCH, SPRAY / SPRAY / SPRAY CHAMBER

885 QUESTION, UNRESOLVED SAFETY

$\mathbf{R}$

$\begin{array}{ll}148 & \text { RACK PIPE } \\ 269 & \text { RACK STORAGE } \\ 106 & \text { RADIATION } \\ 805 & \text { RADIATION MONITOR, PERSONNEL } \\ 810 & \text { RADIATION MONITOR PROCESS } \\ 752 & \text { RADIOACTIVE } \\ 210 & \text { RAIL CAR / CASK CAR } \\ 629 & \text { RAIL / TRACK } \\ 297 & \text { RAILROAD } \\ 242 & \text { RAILROAD, RESPONSIBILITY } \\ 536 & \text { RAIN } \\ 520 & \text { RAN THROUGH, OFF } \\ 999 & \text { RBOF } \\ 055 & \text { RCA } \\ 004 & \text { REACTION, UNCONTROLLED }\end{array}$


TABLE 6

EQUIPMENT CODES (CONT'D)

(Alphabetical Order)

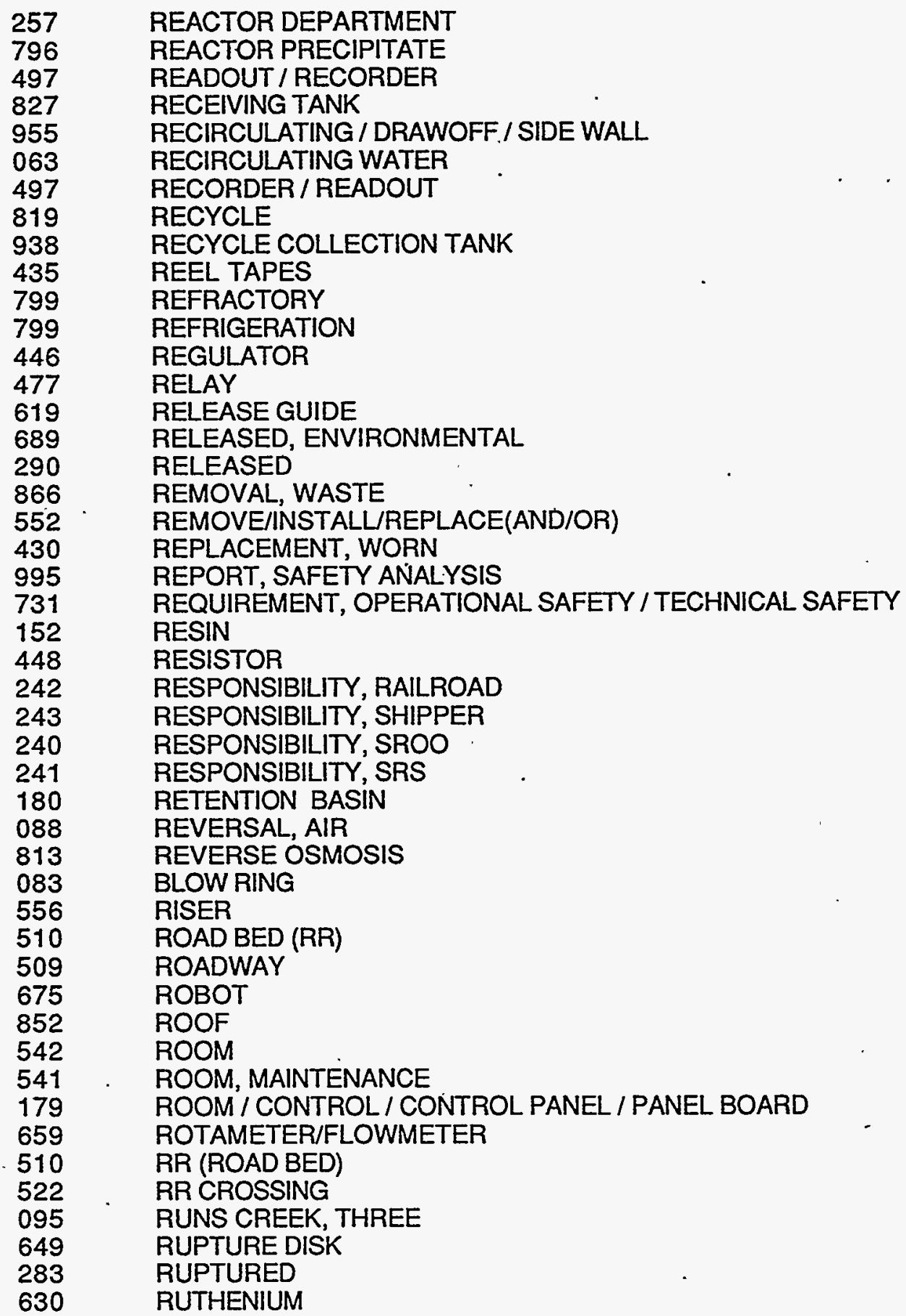


TABLE 6

EQUIPMENT CODES (CONT'D)

(Alphabetical Order)

995 SAFETY ANALYSIS REPORT (SAR)

981

731

SAFETY, EQUIPMENT

SAFETY, OPERATIONAL REQUIREMENTS / TECHNICAL REQUIREMENT

SAFETY SHOWER

731

885

817

737

808

845

620

684

849

523

120

199

778

907

830

266

965

905

121

926

090

086

634

176

957

666

951

265

508

747

256

047

887

SAFETY, TECHNICAL REQUIREMENT / OPERATIONAL REQUIREMENT

SAFETY, UNRESOLVED QUESTION

SALT

SALT-CEMENT MIXING

SALT, FORMATION

SALT, PROCESS CELL

SALVAGE 'YARD

SALVAGE / SREL / DOE ( ERDA ) / FORESTRY / STORES

SAMPLE, ANALYTICAL CELL

SAMPLES

SAMPLING

SAND FILTER

SAND FILTER, BUILDING 294-S

SANITARY LAND FILL

SANITARY WASTE

SATURATION

SCANNER / PRINTER

SCDHEC

SCRAP

SCRAP, PROCESS METAL

SCREENS / STRAINER / FILTERS (GENERAL)

SCREW CONVEYOR

SCRUBBER

SEAL POT

SEAL WATER

SEALER, BAG

SEALS / PACKING / CAULKING

SEALS EQUIPMENT

SECONDARY PACKING

SECURITY

SEDIMENTS

SEEPAGE BASIN

SEISMIC

303

173

774

671

057

924

243

SEGREGATED WATER

SEPARATOR

SERVICE BUILDING, BUILDING 210-S

SERVICES, WACKENHUT

SEWER

SHIELDING

SHIPPER, RESPONSIBILITY 
TABLE 6

EQUIPMENT CODES (CONT'D)

(Alphabetical Order)

SHIPPING CONTAINER SHIPPING PAPERS

SHOP

SHORT CIRCUIT, ELECTRICAL SHOWER, SAFETY

SHUTDOWN

SIDEWALL/ RECIRCULATION / DRAWOFF

SIGHT GLASS

SILICA GEL

SIPHONING

SKID

SKIN CONTAMINATION

SKIP PAN

SLEEVE / BOX / TUBE, ENGINEERED

SLUDGE

SLUDGE RECEIPT AND ADJUSTMENT TANK

SLUICE / FLOOD / DIVERSION, GATE / VALVE

SLUGS

SLURRY MIX EVAPORATOR

SLURRY PUMP

SMEARABLE CONTAMINATION

SMOKE

SMOKING MATERIAL

SNATCHED

SODIUM

SODIUM HYDROXIDE

SODIUM NITRATE

SODIUM NITRATE

SODIUM TETRAPHENYLBORATE

SOIL/CLAY

SOLENOID

SOLID BURNING

SOLVENT

SOLVENT BURNING

SONIC / CONDUCTIVITY PROBE / INTERFACE DETECTOR

SOURCE CHECK

SOURCE, IGNITION, MISC. OR UNKNOWN

SPARGE

SPARKS

SPECTROMETER, MASS

SPEED, EXCESSIVE

SPILL

SPONTANEOUS COMBUSTION

SPECIFIC GRAVITY

SPRAY / QUENCH / SPRAY CHAMBER

SPRAY CHAMBER / SPRAY / SPRAY QUENCH

SPRAY QUENCH / SPRAY / SPRAY CHAMBER

684

SREL / STORES / SALVAGE / FORESTRY / ERDA 


\section{TABLE 6}

EQUIPMENT CODES (CONT'D)

(Alphabetical Order)

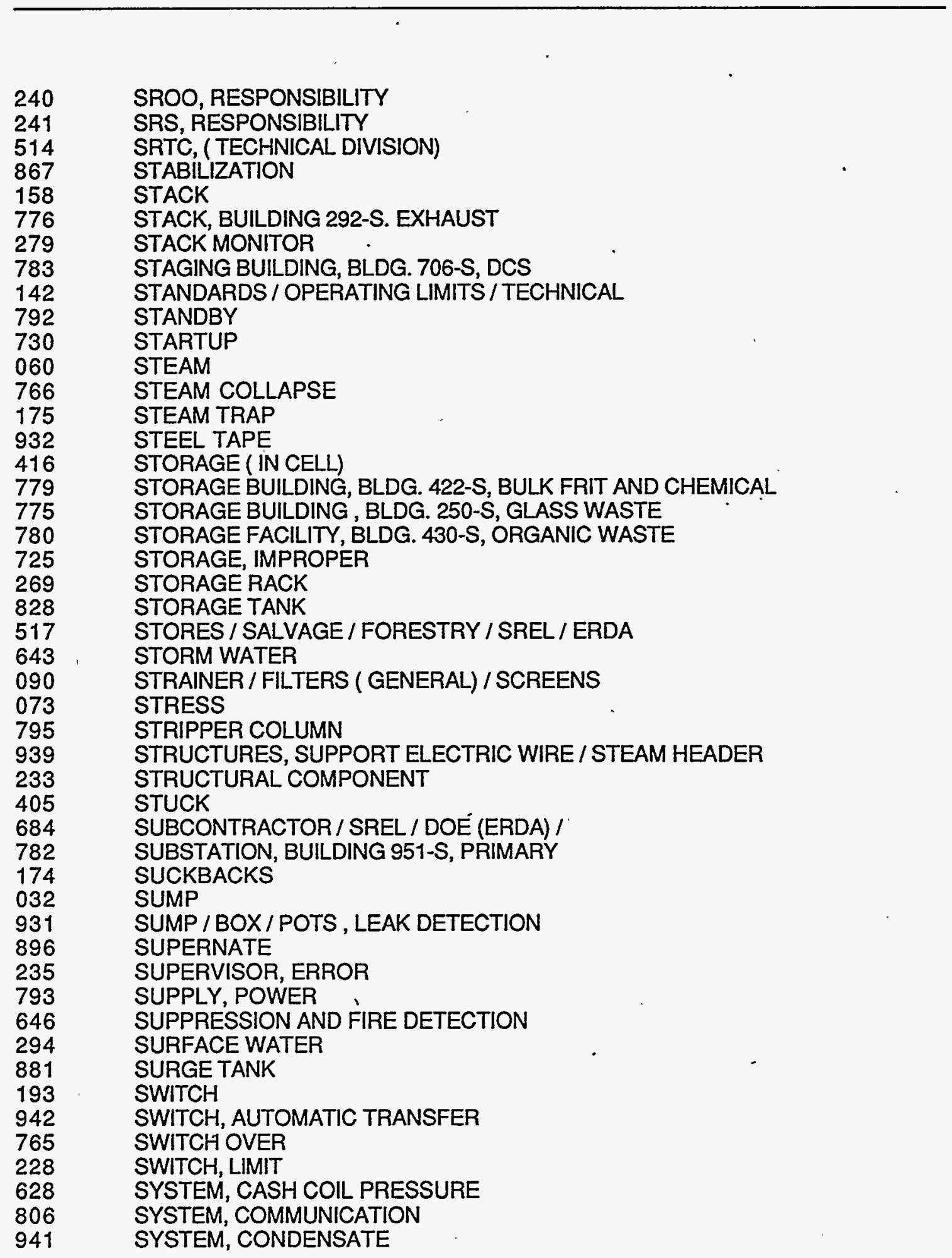


TABLE 6

EQUIPMENT CODES (CONT'D)

(Alphabetical Order)

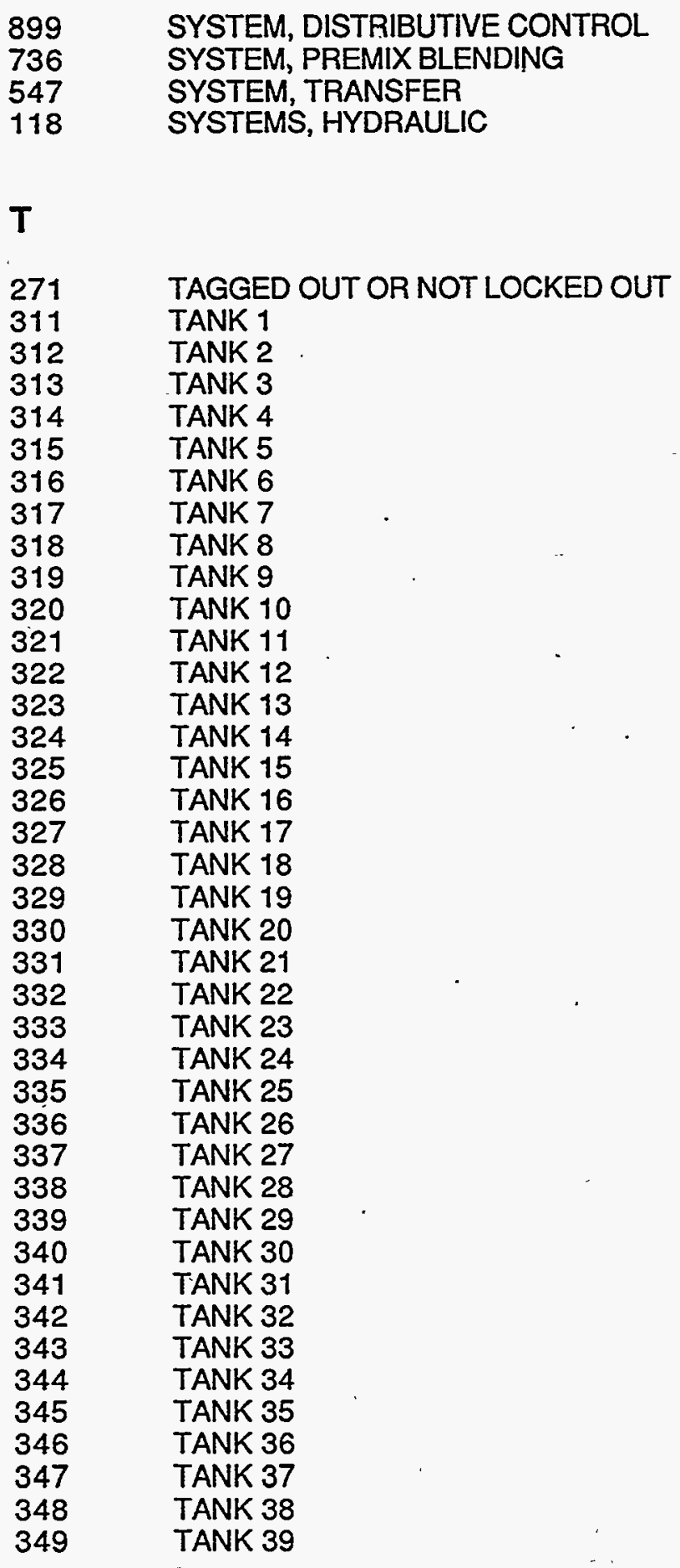


TABLE 6

EQUIPMENT CODES (CONT'D)

(Alphabetical Order)

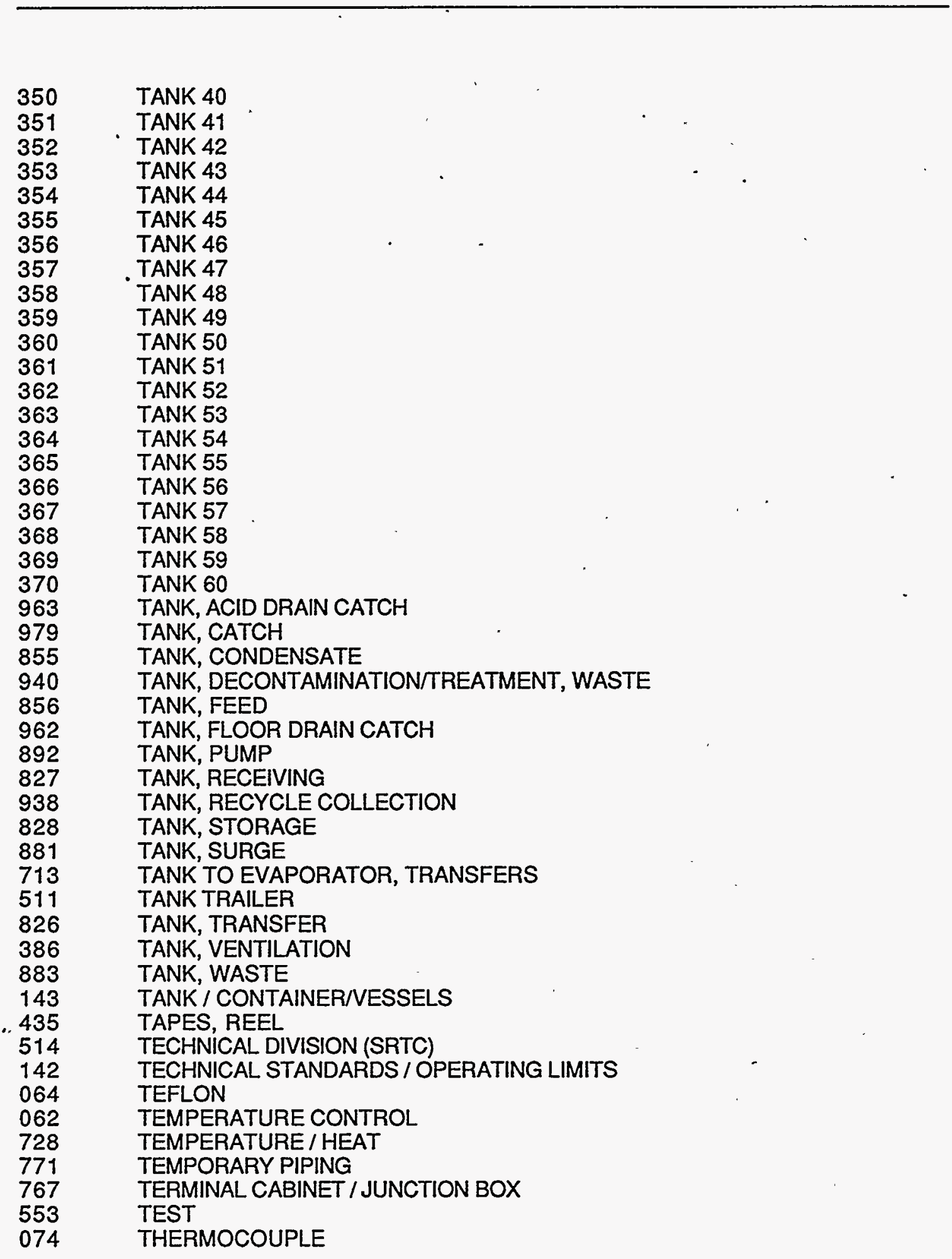


TABLE 6

EQUIPMENT CODES (CONT'D)

(Alphabetical Order)

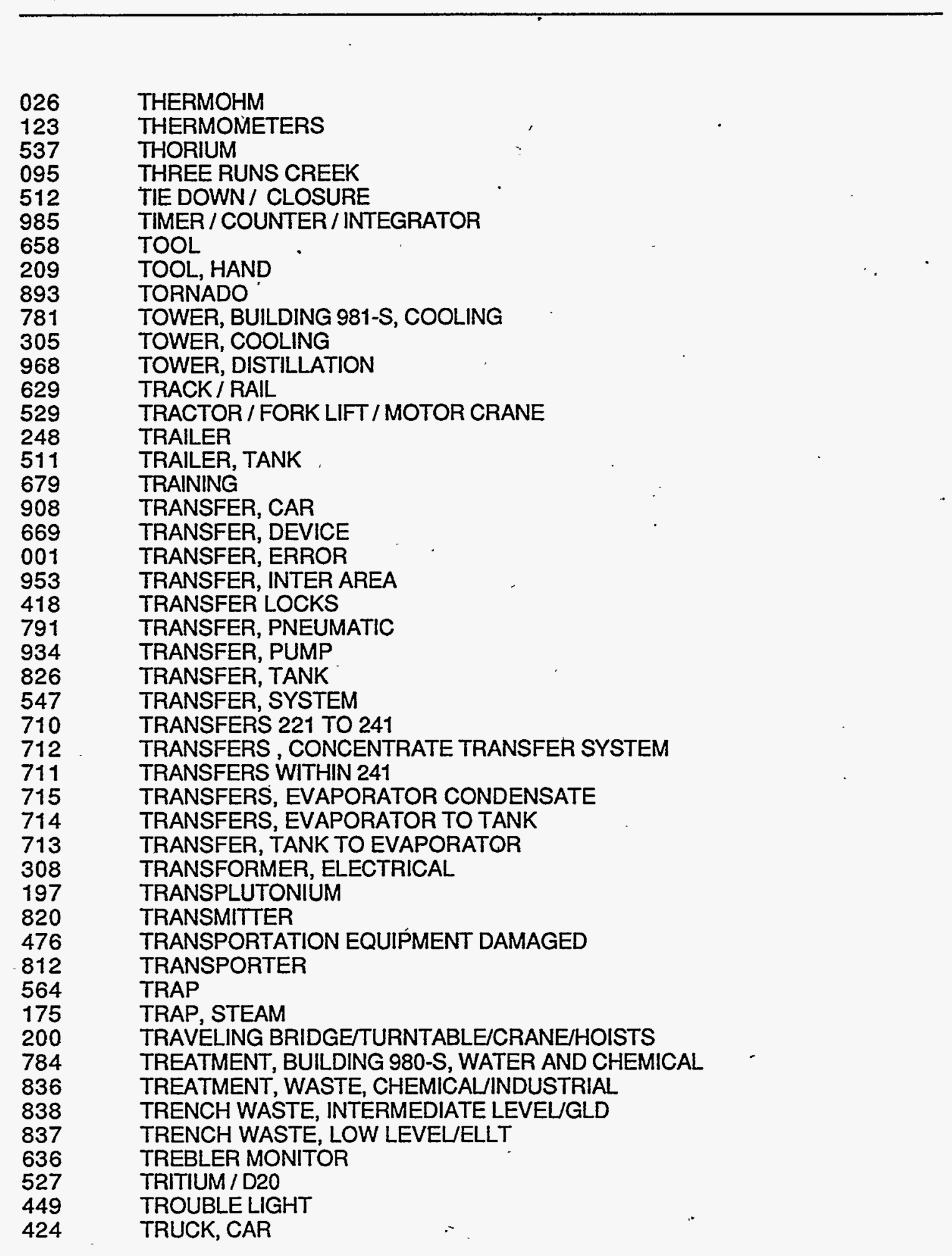


TABLE 6

EQUIPMENT CODES (CONT'D)

(Alphabetical Order)

533

833

203

624

922

296

280

200

720

$\mathbf{U}$

255

724

254

004

162

687

739

223

949

739

683

155

478

479

038

\section{V}

070

422

989

149

983

020

157

984

019

132

555

840

839

842

841

843

TRUCK, PULPWOOD

TRU WASTE, INTERIM STORAGEWCF

TRUNIONS

TUBE, DIP

TUBE/BOX/SLEEVE, ENGINEERED

TUNNEL

TURBIDITY

TURNTABLE/CRANE/HOIST/TRAVELING BRIDGE

TV CAMERA

\author{
UNAUTHORIZED \\ UNAUTHORIZED CONTAINER \\ UNAVAILABLEMISSING \\ UNCONTROLLED REACTION \\ UNDERGROUND \\ UNINTENTIONAL BURIAL \\ UNINTERRUPTIBILE POWER SUPPLY (UPS) \\ UNKNOWN MATERIAL \\ UNPLUG / BLOWDOWN / FLUSH \\ UPS , (UNINTERRUPTIBLE POWER SUPPLY \\ UPTAKE/ABSORPTION \\ UPTAKE/INGESTION \\ UPTAKE/INHALATION \\ UPTAKE/INJECTION \\ URANIUM
}

\author{
VACUUM \\ VACUUM, FURNACE \\ VEGETATION/ALGAE \\ VALENCE ADJUSTMENT \\ VALVE, DIVERSION/FLOOD/SLUICE, GATE \\ VALVE, FAILURE \\ VALVE, GANG \\ VALVE, PIT/BOX \\ VALVING ERROR \\ VAPORIZER / HEATING COIL \\ VAULT \\ VAULT, HAZARDOUS/MIXED, WASTE \\ VAULT, INTERMEDIATE NON TRITIUM WASTE \\ VAULT, INTERMEDIATE WITH TRITIUM WASTE \\ VAULT, LOW LEVEL WASTE \\ VAULT, LONG LIVED WASTE
}


TABLE 6

EQUIPMENT CODES (CONT'D)

(Alphabetical Order)

$\begin{array}{ll}127 & \text { VENDOR } \\ 910 & \text { VENT. VESSEL } \\ 292 & \text { VENTILATION } \\ 919 & \text { VENTILATION, EQUIPMENT WASTE } \\ 387 & \text { VENTILATION, ANNULUS } \\ 386 & \text { VENTILATION, TANK } \\ 260 & \text { VESSEL ENTRY, CLOSED } \\ 143 & \text { VESSEL / TANK I CONTAINER } \\ 480 & \text { VIBRATION } \\ 539 & \text { VIBRATOR } \\ 809 & \text { VIOLATION, LOCK AND TAG } \\ 734 & \text { VIOLATION, OSR } \\ 748 & \text { VIOLATION, PERMIT } \\ 030 & \text { VIOLATION, PROCEDURE } \\ 498 & \text { VISIBILITY PROBLEMS }\end{array}$

W

854 WALL

671 WACKENHUT SERVICES

707 WASHSTATION, EYE

299 WASHER

276 WASTE

277 WASTE, CELL

836 WASTE, CHEMICALINDUSTRIAL, TREATMENT

833 WASTE CERTIFICATION FACILITY/ INTERIM TRU WASTE STORAGE

726 WASTE,COMPACTOR

916 WASTE, ELECTRICALINSTRUMENT, EQUIPMENT

918 WASTE, EXCAVATION (DIRT, ASPHALT, etc.)

909 WASTE, HAZARDOUS

977 WASTE, HIGH LEVEL TRENCH

838 WASTE, INTERMEDIATE LEVEL TRENCH / GCD

839 WASTE, INTERMEDIATE LEVEL NON TRITIUM VAULT

842 WASTE, INTERMEDIATE LEVEL WITH TRITIUM VAULT

832 WASTE, INTERIM HAZARDOUS STORAGE

833

831

. 917

837

841

843

913

866

830

964

775

WASTE, INTERIM TRU, STORAGEWASTE CERTIFICATION FACILITY

WASTE, INTERIM MIXED, TREATMENT/STORAGE

WASTE, JOB CONTROL

WASTE, LOW LEVEL TRENCH / ELLT

WASTE, LOW LEVEL VAULT

WASTE, LONG LIVED VAULT

WASTE, MIXED

WASTE, REMOVAL

WASTE, SANITARY

WASTE, SOLID DISPOSAL FACILITY

WASTE, STORAGE BUILDING, 250-S, GLASS

780

WASTE, STORAGE FACILITY, BLDG. 430-S, ORGANIC 
TABLE 6

EQUIPMENT CODES (CONT'D)

(Alphabetical Order)

WASTE, TREATMENT, BLDG. 980-S, WATER AND CHEMICAL

WASTE; STORAGE ORGANIC

WASTE. TRU

WASTE, PROCESS (PIPE, VALVES, JUMPERS, JETS, ETC.)

WASTE. VENTILATION EQUIPMENT ( FILTERS, ETC.)I

WATER

WATER / CHEMICAL WASTE TREATMENT BLDG. 980-S

WATER, CHILLED

WATER, CHROMATED

WATER, DOMESTIC

WATER, FLUSH

WATER, GROUND

WATER, HAMMER

WATER, PROCESS

WATER, RECALCULATING

WATER, SEGREGATED

WATER, SEAL

WATER, STORM

WATER, SURFACE

WATER, WELL

WEIGHING ERROR

WEIR/OUTLET

WELD

WELD, CELL

WELDER

WELDING

WELL, WATER

WELLS

WIND

WINDOW

WIRING /CORDS/CABLE

WOOD

WORK ORDER DEFICIENCY

WORN REPLACEMENT

WRENCH, IMPACT

\section{$\mathrm{X}$}

Y

620 YARD, SALVAGE

211 YOKE

Z 
TABLE 7

EQUIPMENT CODES

(NUMERICAL ORDER)

$\begin{array}{ll}001 & \text { TRANSFER ERROR } \\ 002 & \text { OVERFLOW } \\ 003 & \text { CHEMICAL ADDITION ERROR } \\ 004 & \text { UNCONTROLLED REACTION } \\ 005 & \text { CRITICALITY POTENTIAL } \\ 006 & \text { SIPHONING } \\ 007 & \text { PLUGGAGE } \\ 008 & \text { LEAKS } \\ 009 & \text { ERUCTATION } \\ 010 & \text { BOILOVER } \\ 011 & \text { BOILOFF } \\ 012 & \text { EXPLOSION } \\ 013 & \text { OVER CONCENTRATION } \\ 014 & \text { COIL LEAKITUBE BUNDLE LEAK } \\ 015 & \text { INSTRUMENT MALFUNCTION } \\ 016 & \text { CORROSION } \\ 017 & \text { JET } \\ 018 & \text { PRECIPITATION } \\ 019 & \text { VALVING ERROR } \\ 020 & \text { VALVE FAILURE } \\ 021 & \text { FOAMING } \\ 023 & \text { DEMISTER } \\ 024 & \text { FLANGEIGASKET } \\ 025 & \text { JUMPER } \\ 026 & \text { THERMOHM } \\ 030 & \text { PROCEDURAL VIOLATION } \\ 032 & \text { SUMP } \\ 034 & \text { CONDENSER } \\ 036 & \text { PROCESS WATER } \\ 037 & \text { ACTIVITY } \\ 038 & \text { URANIUM } \\ 039 & \text { ORGANIC } \\ 041 & \text { NATURAL PHENOMENON } \\ 042 & \text { ICEIFROZEN } \\ 043 & \text { COLUMN FLOODING } \\ 044 & \text { NITRICACID } \\ 045 & \text { COOLING WATER } \\ 046 & \text { FOUR MILE CREEK } \\ 047 & \text { SEEPAGE BASIN } \\ 048 & \text { CONTAMINATION, FACILITY OR EQUIPMENT } \\ 049 & \text { ACID ABSORBER } \\ 050 & \text { BREAKTHROUGH } \\ 051 & \text { SILICA GEL } \\ 052 & \text { PIPING ERROR } \\ 053 & \text { OPERATING ERROR } \\ 054 & \text { PROCEDURAL DIFFICULTY } \\ 055 & \text { RCA } \\ 056 & \text { PERSONAL EXPOSURE } \\ & \\ 0.9\end{array}$


TABLE 7

EQUIPMENT CODES

(NUMERICAL ORDER)

\begin{tabular}{|c|c|}
\hline $\begin{array}{l}057 \\
058 \\
059 \\
060 \\
062 \\
063 \\
064 \\
065 \\
066 \\
068 \\
069 \\
070 \\
071 \\
072 \\
073 \\
074 \\
075 \\
076 \\
077 \\
079 \\
080 \\
082 \\
083 \\
084 \\
085 \\
086 \\
087 \\
088 \\
089 \\
090 \\
091 \\
092 \\
093 \\
095 \\
096 \\
097 \\
098 \\
100 \\
101 \\
102 \\
103 \\
104 \\
105 \\
106 \\
107 \\
109 \\
110\end{array}$ & 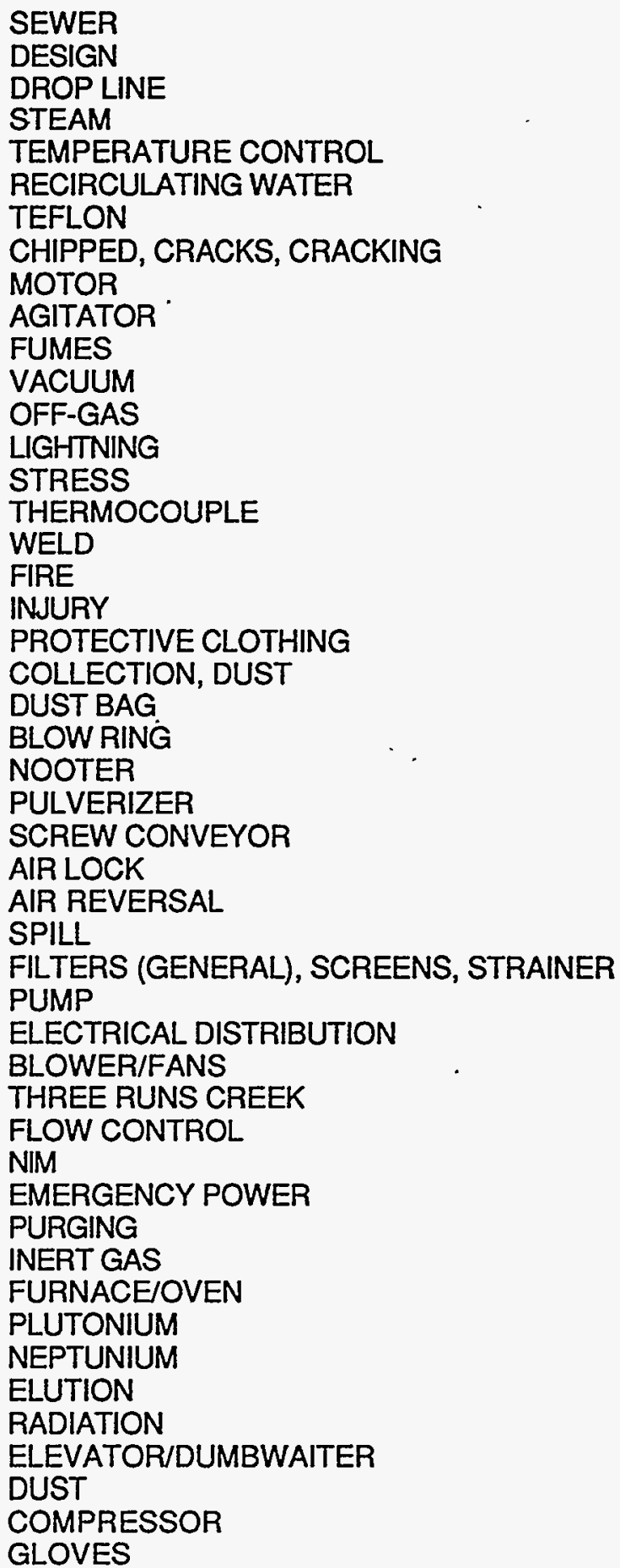 \\
\hline
\end{tabular}


TABLE 7

EQUIPMENT CODES

(NUMERICAL ORDER)

$\begin{array}{ll}112 & \text { DEFORMATION } \\ 114 & \text { EXHAUSTER } \\ 115 & \text { DECOMPOSITIONIDECAY } \\ 116 & \text { JACKET LEAK } \\ 117 & \text { CRUCIBLE } \\ 118 & \text { HYDRAULIC SYSTEMS } \\ 119 & \text { DEIONIZER, ION EXCHANGE COLUMNS } \\ 120 & \text { SAMPLING } \\ 121 & \text { SCRAP } \\ 123 & \text { THERMOMETERS } \\ 124 & \text { ANALYTICAL } \\ 125 & \text { IMPURITIES } \\ 126 & \text { DRAIN } \\ 127 & \text { VENDOR } \\ 128 & \text { EQUIPMENT } \\ 129 & \text { MALFUNCTION/FAULTY } \\ 130 & \text { ACCOUNTABILITY } \\ 131 & \text { MOTOR GENERATOR } \\ 132 & \text { HEATING COILNAPORIZER } \\ 133 & \text { CAUSTIC ALKALI } \\ 135 & \text { SPARGE } \\ 136 & \text { HEADER } \\ 137 & \text { CALCULATIONAL ERROR } \\ 138 & \text { BLANK } \\ 141 & \text { PUNCTURE } \\ 142 & \text { TECHNICAL STANDARDS/OPERATING LIMITS } \\ 143 & \text { VESSELTANKICONTAINER } \\ 144 & \text { PRESSURE } \\ 145 & \text { HUMIDITY } \\ 146 & \text { SHUTDOWN } \\ 147 & \text { EMBEDDED PIPE } \\ 148 & \text { RACK PIPE } \\ 149 & \text { VALENCE ADJUSTMENT } \\ 150 & \text { HUT } \\ 151 & \text { BREATHING AIR } \\ 152 & \text { RESIN } \\ 153 & \text { CONTAMINATION, AIRBORNE } \\ 154 & \text { PERSONNEL } \\ 155 & \text { UPTAKEINGESTION } \\ 156 & \text { INHALATION } \\ 157 & \text { GANG VALVE } \\ 158 & \text { STACK } \\ 159 & \text { COMMUNICATIONS, PHONE } \\ 161 & \text { FLOODED } \\ 162 & \text { UNDERGROUND } \\ 163 & \text { EVAPORATOR (GENERAL) } \\ 164 & \text { DECANT } \\ 165 & \text { PIPE } \\ & \end{array}$




\section{TABLE 7}

\section{EQUIPMENT CODES \\ (NUMERICAL ORDER)}

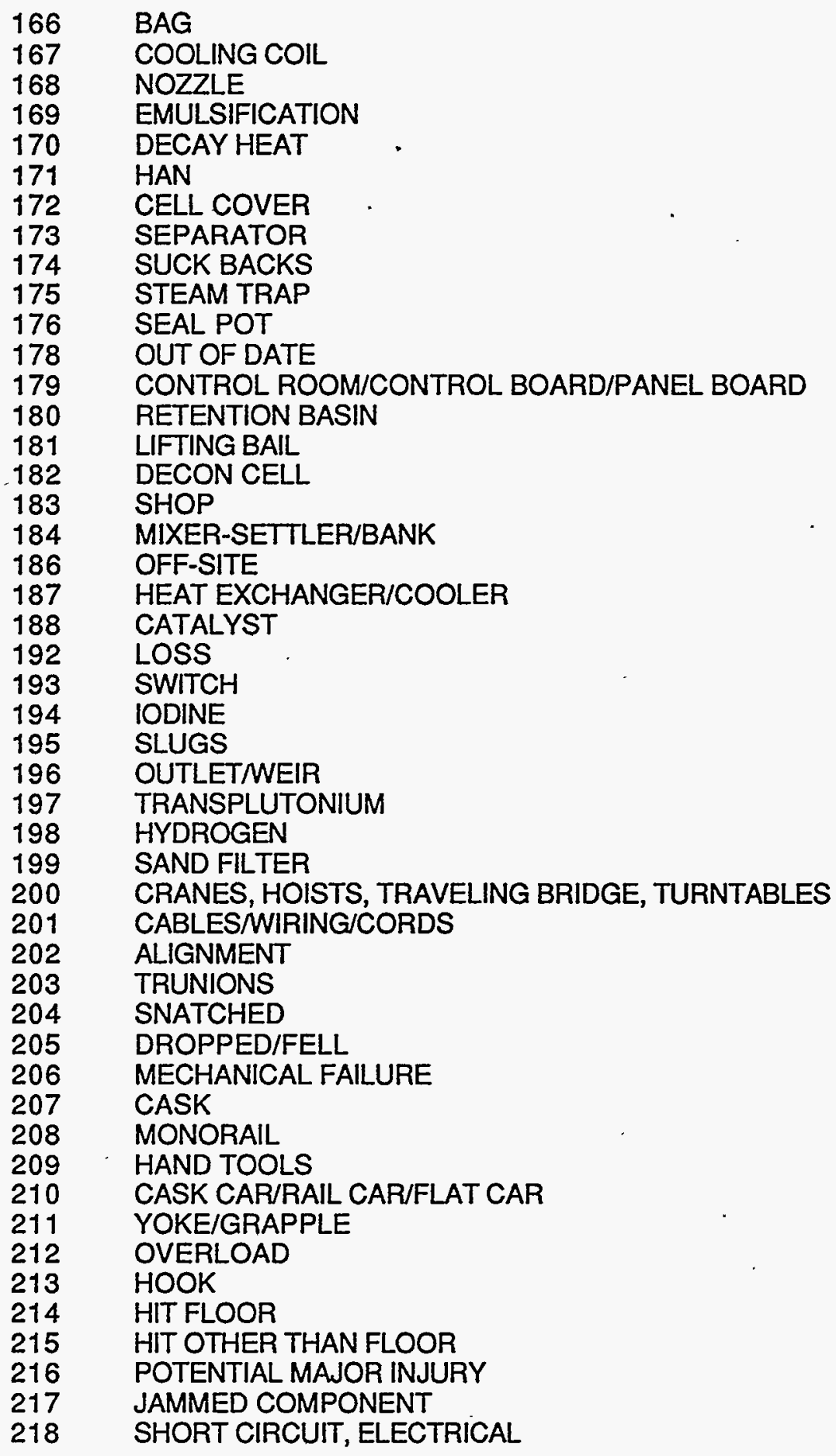


TABLE 7

EQUIPMENT CODES

(NUMERICAL ORDER)

219 MECHANICAL DAMAGE

221 INADEQUATE FUNCTION

222 BREAKER

223

224

225

226

227

228

229

230

231

232

233

234

UNKNOWN MATERIAL

ELECTRICAL CONTACT

FAULTY COMPONENT

PROTECTIVE DEVICE

BRAKE

LIMIT SWITCH

PROTECTIVE DEVICE MISSING

FUEL

BROKEN/DAMAGED

BUNDLETTUBE BUNDLE-

STRUCTURAL COMPONENT

BURIAL GROUND

SUPERVISOR ERROR

INCOMPLETE JOB

INCORRECT POSITION

INSECURE

SHIPPING CONTAINER

RESPONSIBILITY, SROO

RESPONSIBILITY, SRP

RESPONSIBILITY, RAILROAD

RESPONSIBILITY, SHIPPER

CAME APART

TRAILER

IMPROPER PACKAGING

ERRORS

SHIPPING PAPERS

DIMENSIONS

MISSING / UNAVAILABLE

UNAUTHORIZED

SEDIMENTS

REACTOR DEPARTMENT

MATERIAL LOST

LOCKED OUT

CLOSED VESSEL ENTRY

HOSE

SIGHT GLASS

LIGHTS

WATER

EQUIPMENT SEALS

SATURATION

STORAGE RACK

HM ALARM 
TABLE 7

EQUIPMENT CODES

(NUMERICAL ORDER)

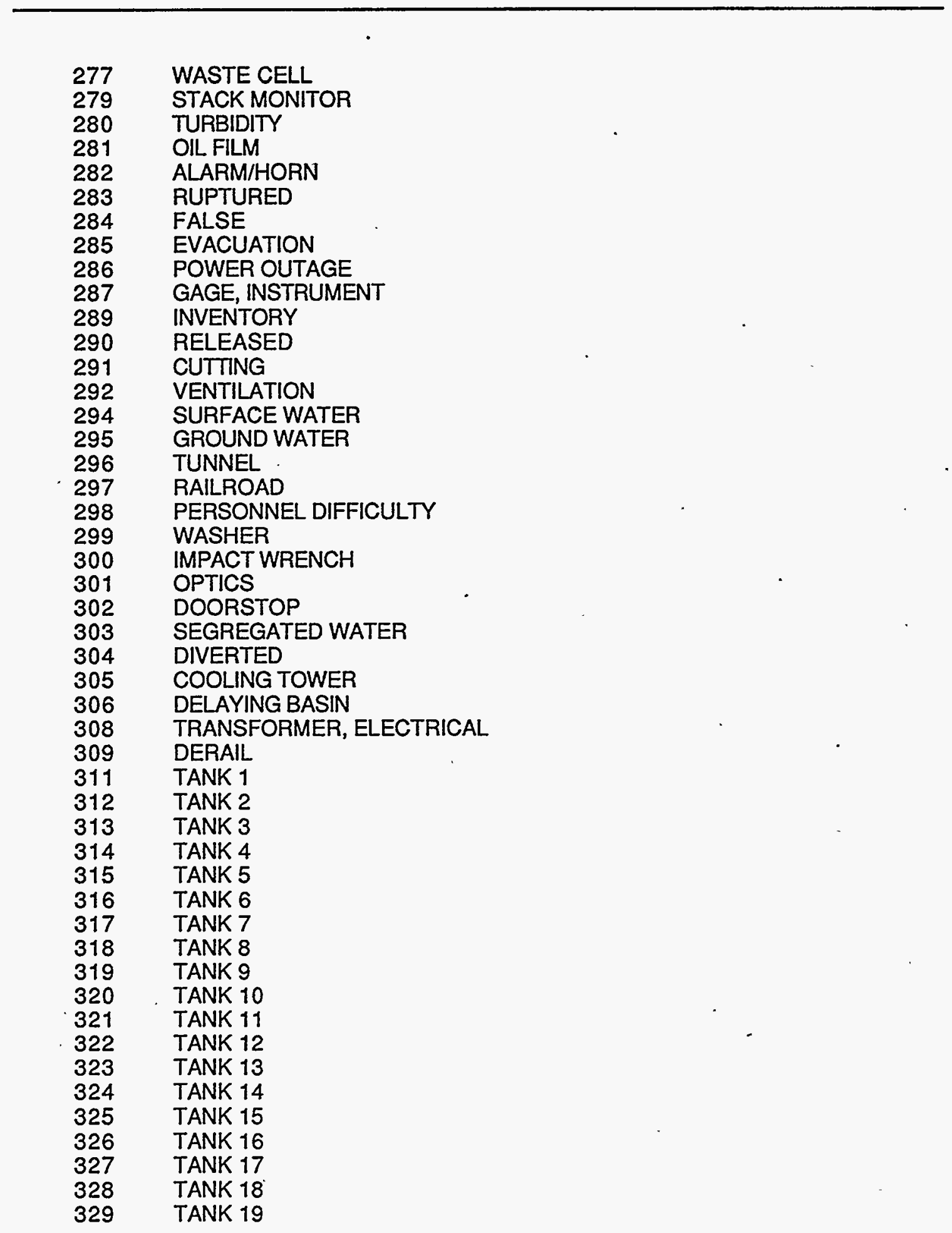


TABLE 7

EQUIPMENT CODES

(NUMERICAL ORDER)

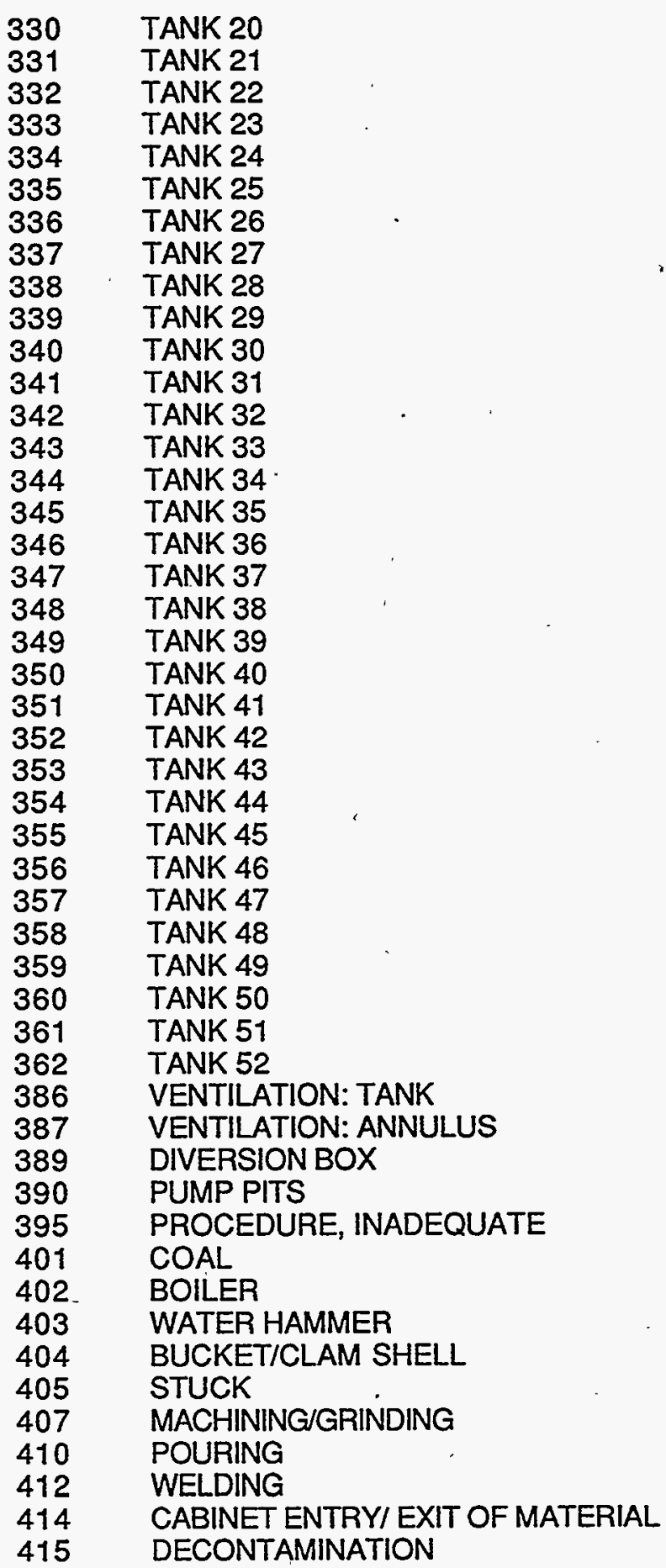


TABLE 7

EQUIPMENT CODES

(NUMERICAL ORDER)

\begin{tabular}{|c|c|}
\hline $\begin{array}{l}416 \\
417 \\
418 \\
419 \\
422 \\
425 \\
426 \\
427 \\
428 \\
429 \\
430 \\
431 \\
432 \\
433 \\
435 \\
436 \\
438 \\
439 \\
440 \\
441 \\
442 \\
443 \\
444 \\
445 \\
446 \\
447 \\
448 \\
449 \\
450 \\
451 \\
452 \\
453 \\
454 \\
455 \\
456 \\
457 \\
458 \\
459 \\
460 \\
462 \\
463 \\
464 \\
465 \\
466 \\
467 \\
468 \\
469 \\
470\end{array}$ & 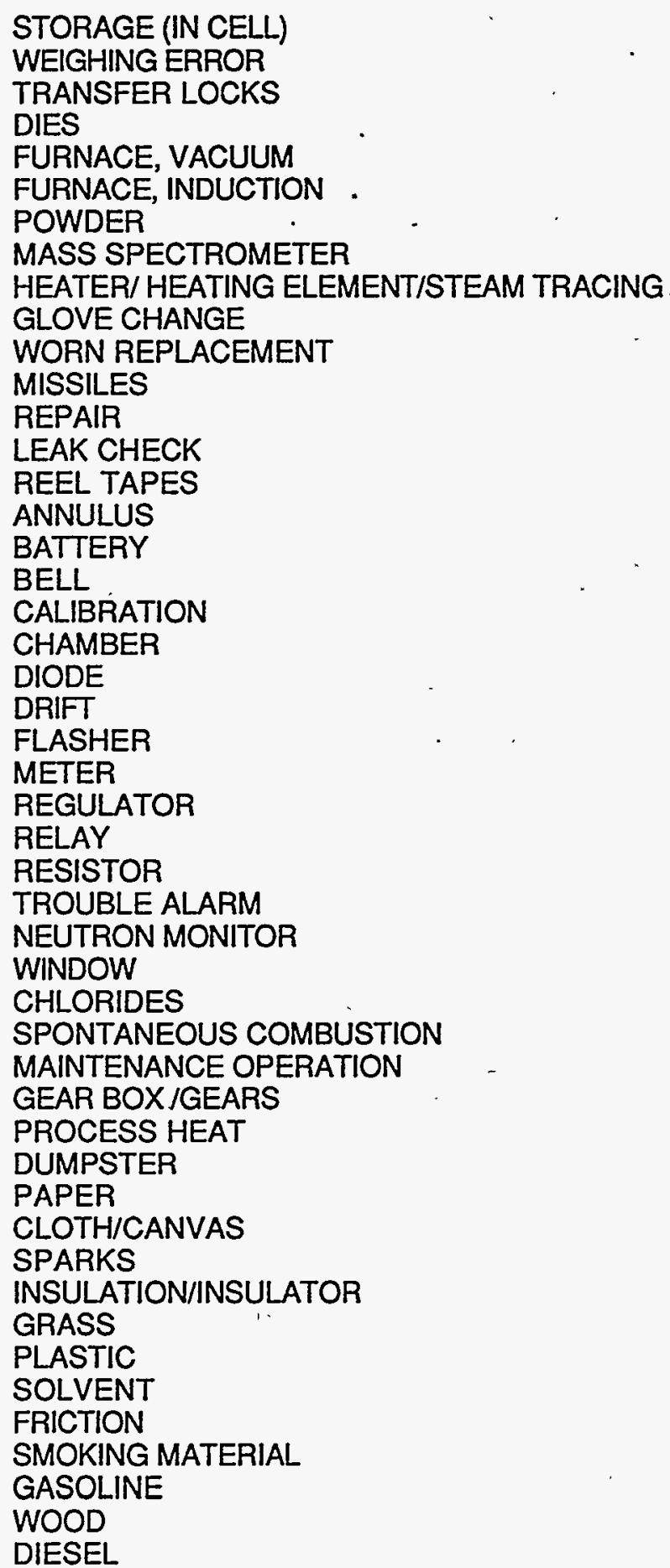 \\
\hline
\end{tabular}


TABLE 7

EQUIPMENT CODES

(NUMERICAL ORDER)

471 METAL

472 GAS

473 MISC. OR UNKNOWN MATL BURNED

474 MISC. OR UNKNOWN IGNITION SOURCE

475 ELECTRICAL EQUIPMENT DAMAGED

476 TRANSPORTATION EQUIPMENT DAMAGED

477 REFRIGERATION

478 UPTAKE/INHALATION

479 UPTAKE/INJECTION

480 VIBRATION

481 COMPUTER

482 COMPRESSED AIR

483 PAVEMENT

484 GRATE

485 REFRACTORY

486 COAL HANDLING EQUIPMENT

489 WELLS

490 DUCT

491 BALANCE

492 GENERATOR/CHARGER

493 PRECIPITATOR

494 COATING (LINING)/LINER

496 INSTRUMENT AIR

497 RECORDER/READOUT

498 VISIBILITY PROBLEM

500 LIQÜID LEVEL

501 BEARINGS,

502

503

504

505

506

507

508

509

510

511

512

513

514

517

518

519

520

521

522

523

524

AMMONIA COMPOUNDS

FLUORINE COMPOUNDS

COLLISION

DRUMS, CANS

INADEQUATE MONITORING .

PRIMARY PACKAGING

SECONDARY PACKAGING

ROADWAY

ROAD BED (RR)

TANK TRAILER

TIE DOWN/ CLOSURE

CHOCKS

TECHNICAL DIVISION (SRTC)

STORES, SALVAGE, FORESTRY, SREL, ERDA

EXCESSIVE SPEED

LOCOMOTIVE

RAN THROUGH, OFF

DEBRIS

RR CROSSING

SAMPLES

TRUCK, CAR 
WSRC-TR-95-0173

Page 51

TABLE 7

EQUIPMENT CODES

(NUMERICAL ORDER)

$\begin{array}{ll}525 & \text { LOSS OF LOAD } \\ 527 & \text { TRITIUM, D2O } \\ 528 & \text { OVERTURN } \\ 529 & \text { FORK LIFTMOTOR CRANETRACTOR/LIFT TRUCK } \\ 530 & \text { JACK KNIFE } \\ 531 & \text { SKID } \\ 532 & \text { FATALITIES } \\ 533 & \text { PULPWOOD TRUCK } \\ 535 & \text { INATTENTION, BLACKOUT } \\ 536 & \text { RAIN } \\ 537 & \text { THORIUM } \\ 538 & \text { MISLABELED } \\ 539 & \text { VIBRATOR } \\ 541 & \text { MAINTENANCE ROOM } \\ 542 & \text { ROOM } \\ 543 & \text { INADEQUATE } \\ 544 & \text { LIMITEXCEEDED } \\ 545 & \text { INTERLOCK } \\ 546 & \text { TRAP } \\ 547 & \text { TRANSFER SYSTEM } \\ 548 & \text { CONTAMINATION, SKIN } \\ 549 & \text { MONITORING } \\ 550 & \text { DRILLDRILLING } \\ 551 & \text { INSPECTION } \\ 552 & \text { INSTALL, REPLACE (ANDIOR REMOVE) } \\ 553 & \text { TEST } \\ 554 & \text { AMERICIUM } \\ 555 & \text { VAULT } \\ 556 & \text { RISER } \\ 558 & \text { CONSTRUCTION DIVISION } \\ 559 & \text { METEOROLOGICAL INSTRUMENTS } \\ 574 & \text { BROKEN, EQUIPMENT } \\ 582 & \text { BIOASSAY AND/OR CHEST COUNT } \\ 619 & \text { RELEASE GUIDE } \\ 620 & \text { SALVAGE YARD } \\ 622 & \text { AIR, PROCESS } \\ 623 & \text { DAMPER } \\ 624 & \text { DIPTUBE } \\ 625 & \text { MOTOR CONTROL CENTER } \\ 626 & \text { DOOR } \\ 627 & \text { MANIPULATOR } \\ 628 & \text { CASH COIL PRESSURE SYSTEM } \\ 629 & \text { RAILTRACK } \\ 630 & \text { RUTHENIUM } \\ 631 & \text { WIND } \\ 632 & \text { DEHUMIDIFIER } \\ 633 & \text { CONCRETE } \\ 634 & \text { SCRUBBER } \\ & \end{array}$


TABLE 7

EQUIPMENT CODES

(NUMERICAL ORDER)

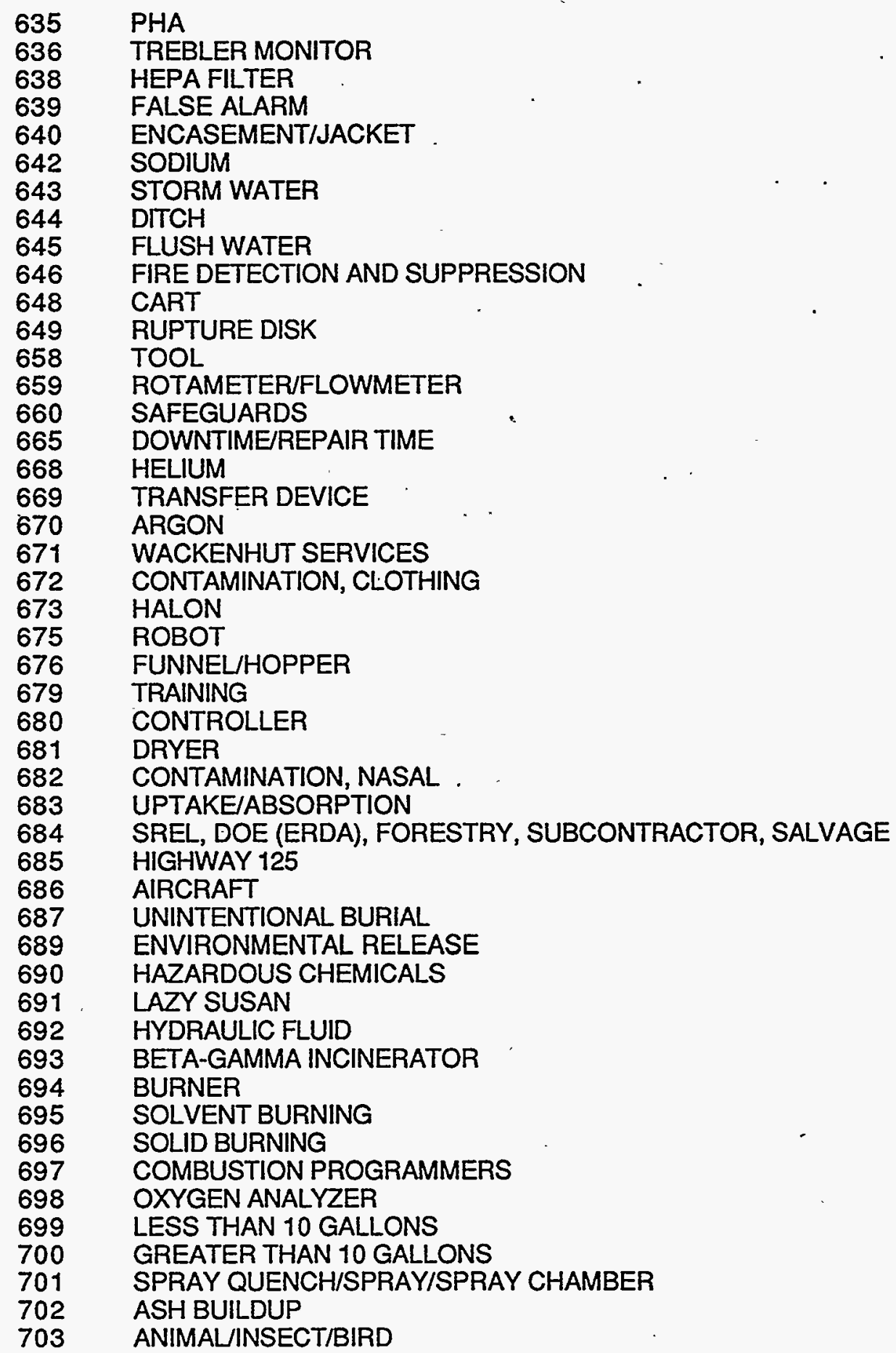




\section{EQUIPMENT CODES}

(NUMERICAL ORDER)

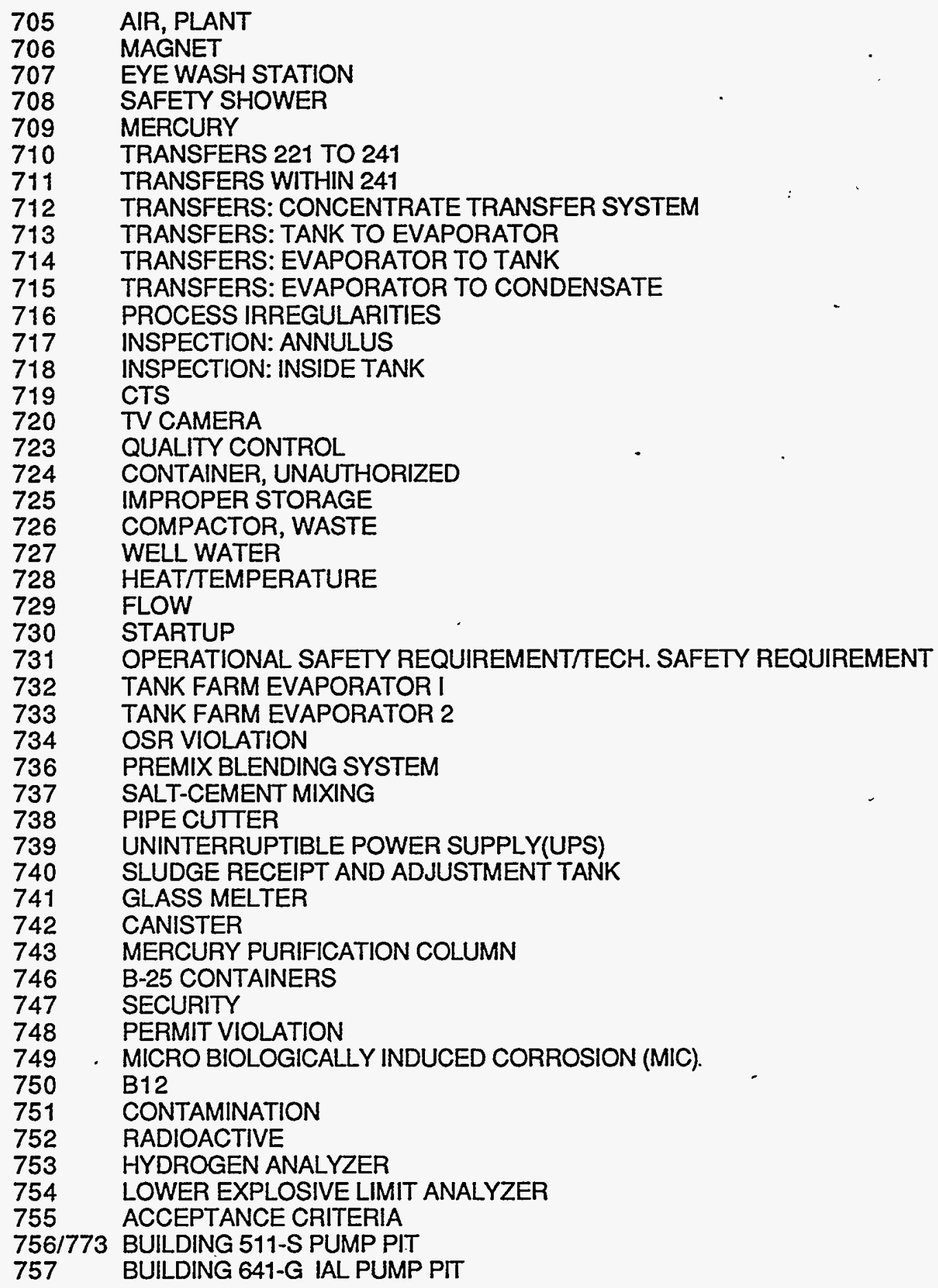


TABLE 7

EQUIPMENT CODES

(NUMERICAL ORDER)

\begin{tabular}{|c|c|}
\hline $\begin{array}{l}770 \\
771 \\
772 \\
773 / 756 \\
774 \\
775 \\
776 \\
777 \\
778 \\
779 \\
780 \\
781\end{array}$ & 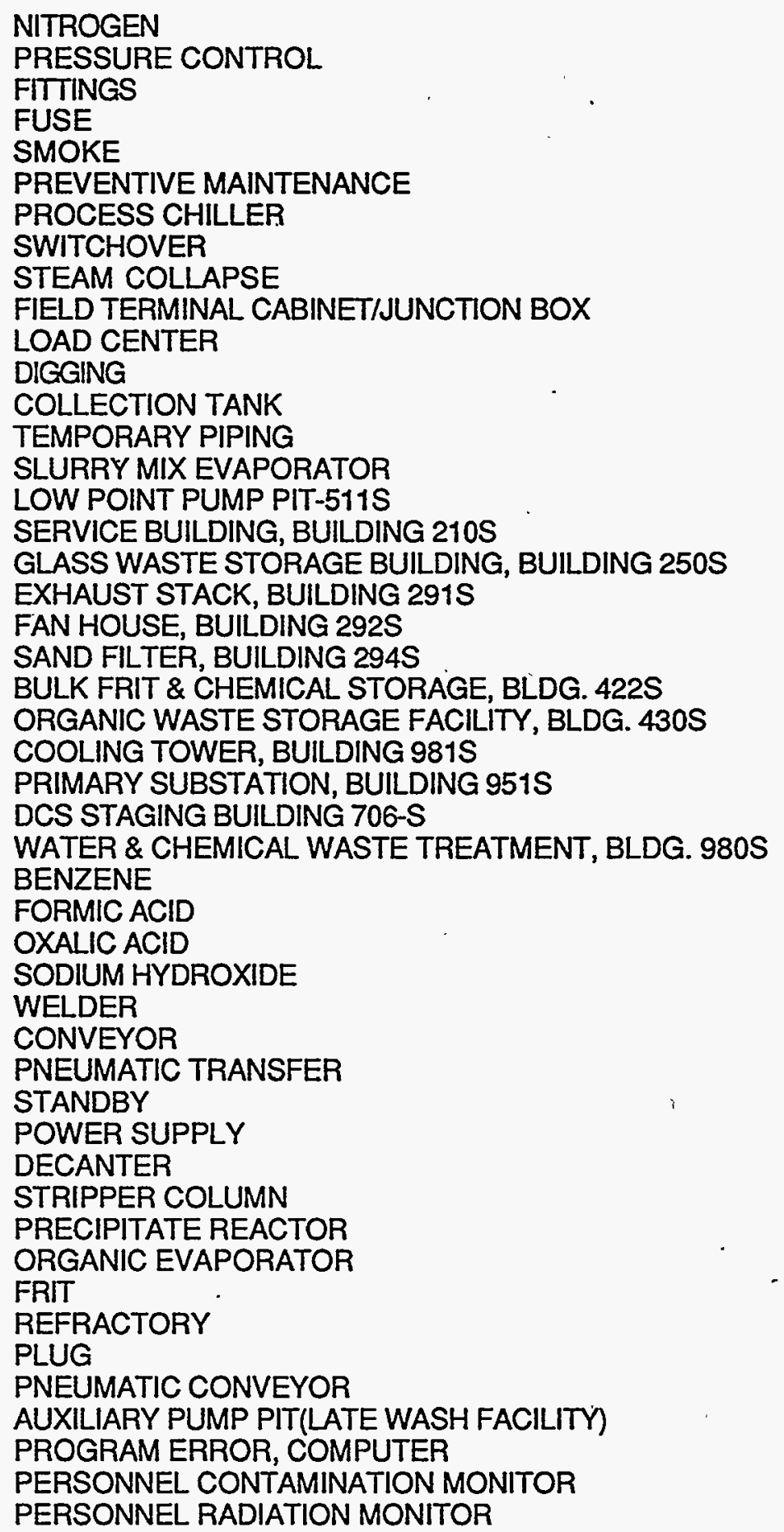 \\
\hline
\end{tabular}


TABLE 7

EQUIPMENT CODES

(NUMERICAL ORDER)

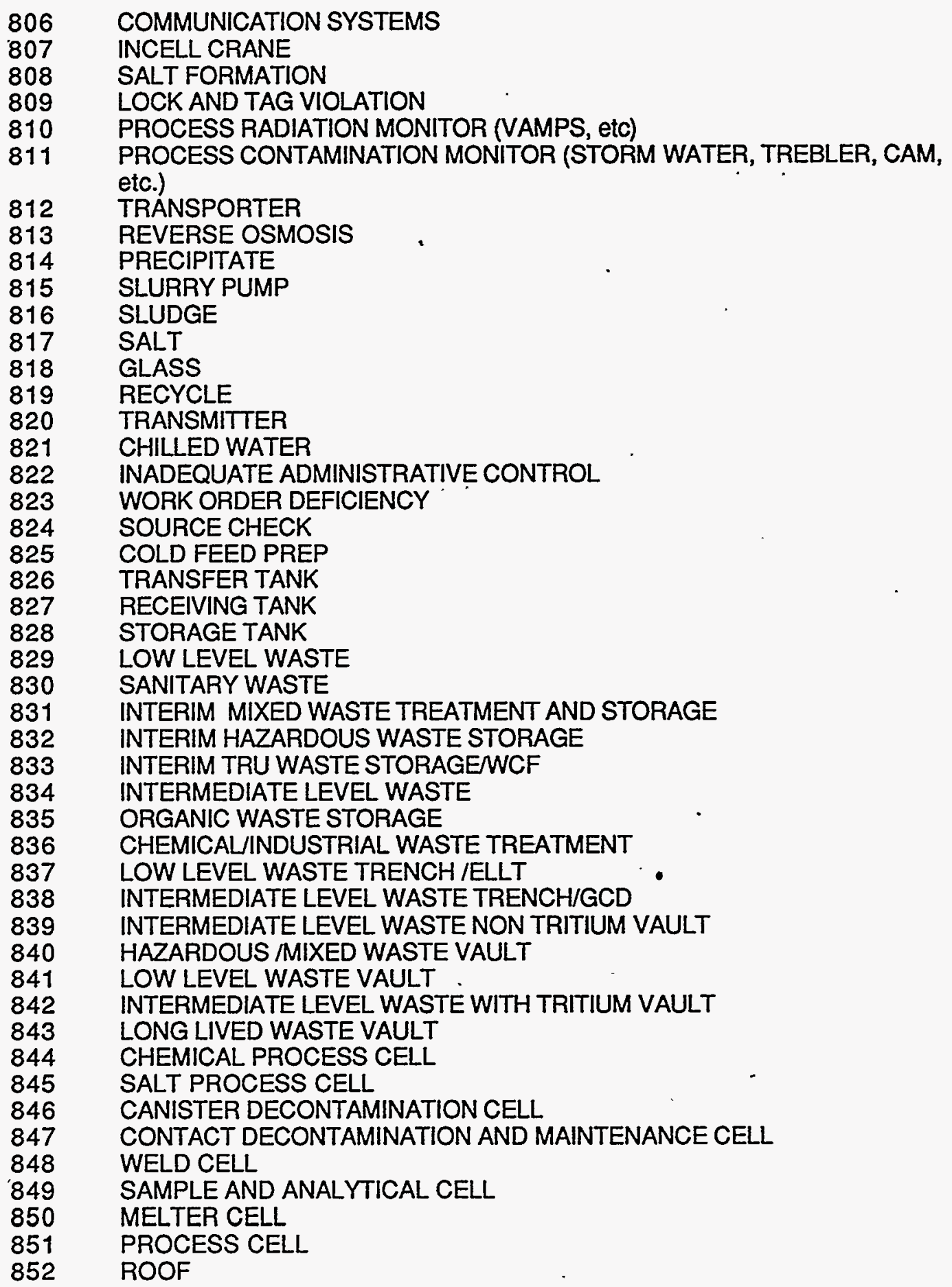


TABLE 7

EQUIPMENT CODES

(NUMERICAL ORDER)

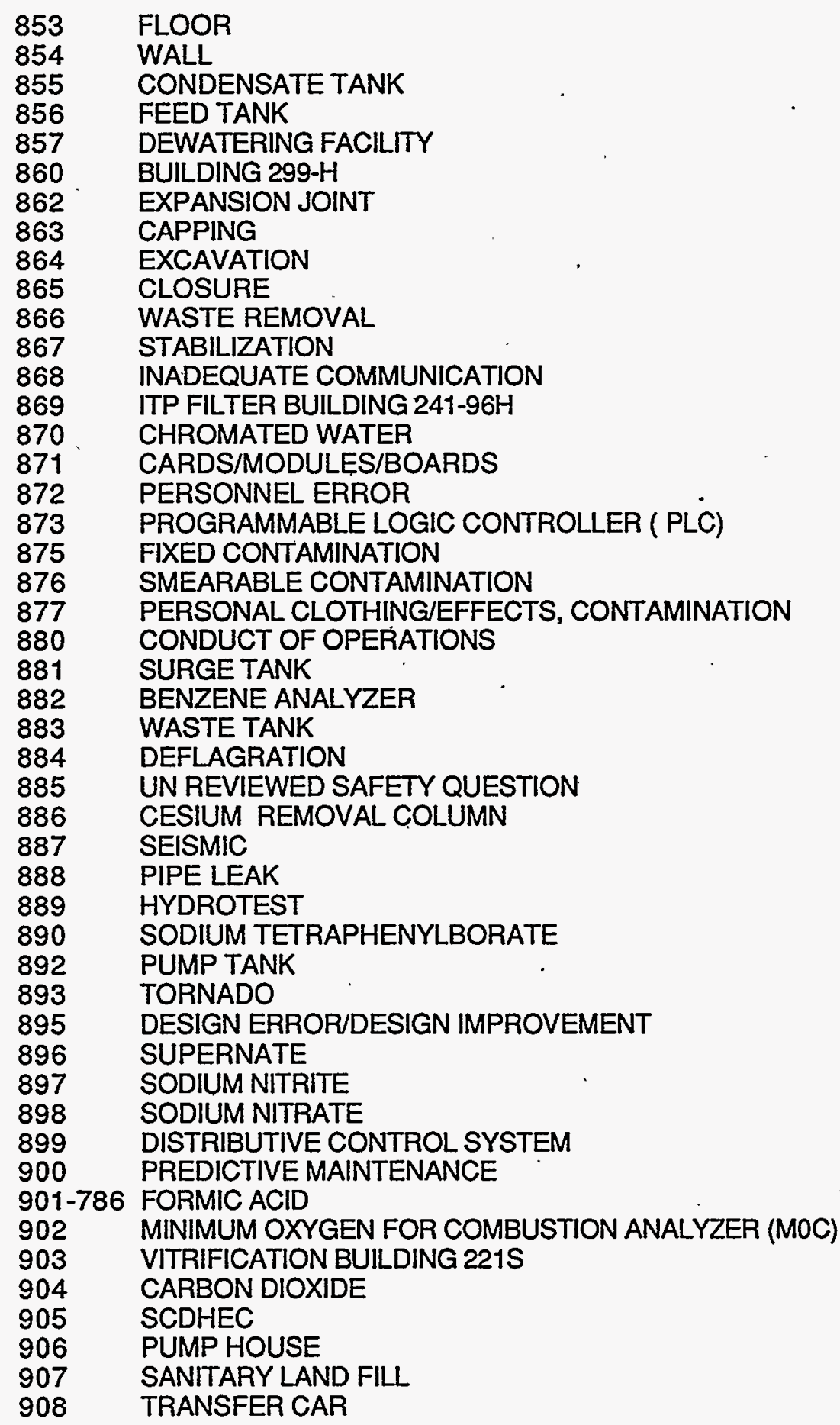


TABLE 7

EQUIPMENT CODES

(NUMERICAL ORDER)

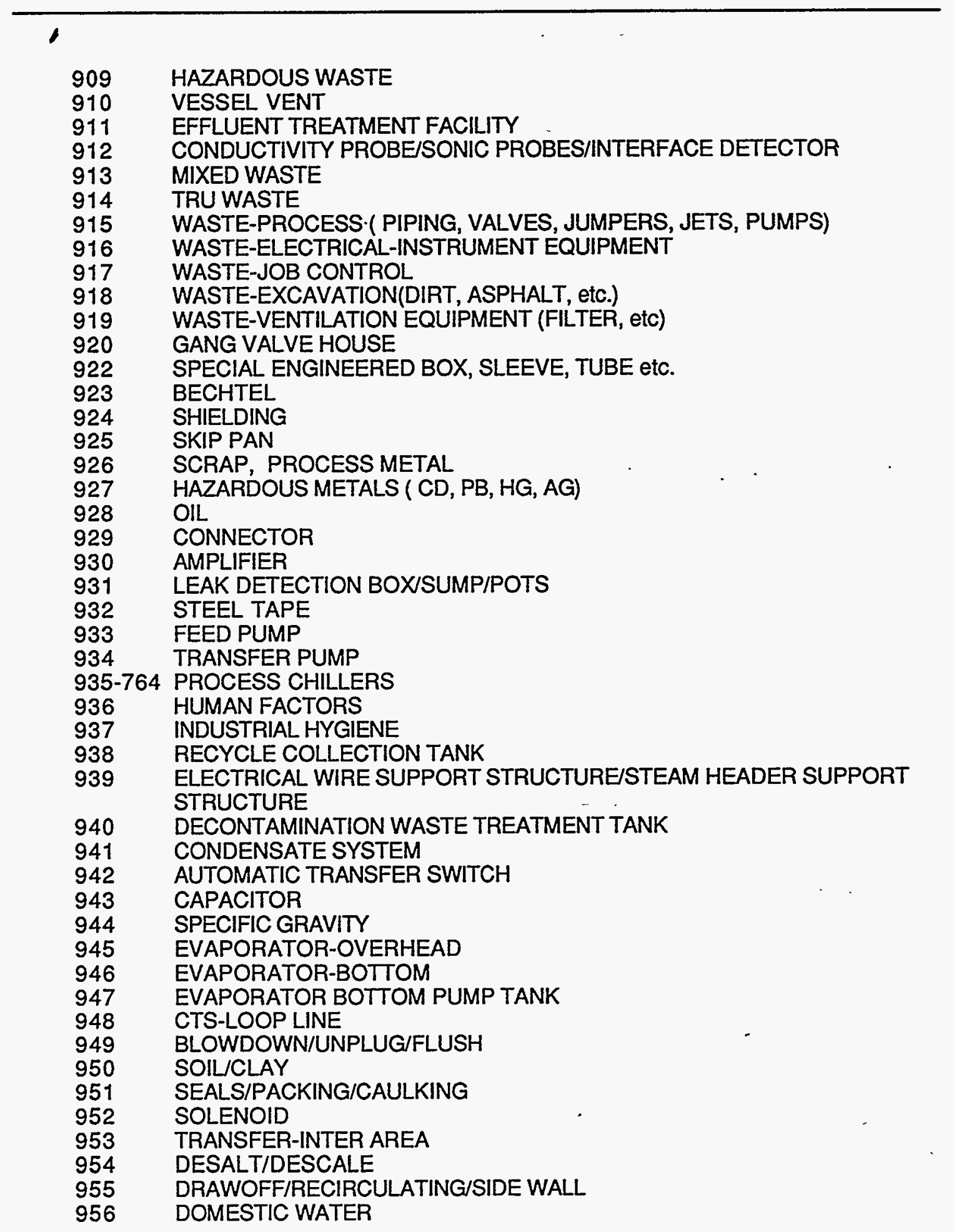


TABLE 7

EQUIPMENT CODES

(NUMERICAL ORDER)

$\begin{array}{ll}957 & \text { SEAL WATER } \\ 958 & \text { HOT.DECONTAMINATION WASTE HEADER } \\ 959 & \text { WARM DECONTAMINATION WASTE HEADER } \\ 960 & \text { FORMIC ACID WASTE HEADER } \\ 961 & \text { MERCURY TRANSFER HEADER } \\ 962 & \text { FLOOR DRAIN CATCH TANK } \\ 963 & \text { ACID DRAIN CATCH TANK } \\ 964 & \text { SOLID WASTE DISPOSAL FACILITY } \\ 965 & \text { PRINTER/SCANNER } \\ 966 & \text { CIRCUIT } \\ 967 & \text { PROCESS HAZARD } \\ 968 & \text { DISTILLATION TOWER } \\ 977 & \text { HIGHLEVEL WASTE TRENCH } \\ 978 & \text { IMPELLER } \\ 979 & \text { CATCH TANK } \\ 980 & \text { BELT } \\ 981 & \text { SAFETY EQUIPMENT } \\ 982 & \text { 21OZ BUILDING } \\ 983 & \text { DIVERSION/FLOOD/SLUICE, GATE/VALVE } \\ 984 & \text { VALVE PIT/BOX } \\ 985 & \text { TIMER/COUNTER/NTEGRATOR } \\ 986 & \text { LATE WASH FACILITY } \\ 988 & \text { AIR CONDITION } \\ 989 & \text { VEGETATION/ALGAE } \\ 990 & \text { INTERMEDIATE ABOVE GROUND STORAGE } \\ 991 & \text { EROSION } \\ 992 & \text { BARRICADE/FENCE/ENCLOSURE/DIKE } \\ 993 & \text { DETECTOR } \\ 994 & \text { LYSIMETER } \\ 995 & \text { SAFETY ANALYSIS REPORT(SAR) } \\ 996 & \text { DIAPHRAGM } \\ 997 & \text { DEMOLITION/DECOMMISSION } \\ 998 & \text { ASBESTOS } \\ 999 & \text { RBOF } \\ & \\ \end{array}$


TABLE 8

EQUIPMENT CODE

(CATEGORY ORDER INDEX)

\section{ADMINISTRATIVE}

- Plant Organizations Terms

- Emergency Response/Criticality Terms

- Procedure Terms

- Human Factor Terms

- Vendor Related Terms

2. CONTAINERS: SHIPPING AND STORAGE

- Generic Terms

- Defined Terms

3. COMMUNICATION

4. ELECTRICAL

- Generic Terms

- Defined Terms

- Emergency Power Terms

- Equipment Terms

5. ENVIRONMENTAL

- Generic Terms

- Defined Terms

- Air/Effluent Monitor Systems Terms

- Environmental Releases Terms

6. ERRORS/FAILURES/ROOT CAUSES

- Generic Terms

- Defined Terms

7. FIRE

- Generic Terms-General

- Generic Terms-Combustible Types 
TABLE 8

EQUIPMENT CODE(cont'd)

(CATEGORY ORDER INDEX)

\section{GENERIC TERMS-GENERAL}

- Action Terms

- Equipment Terms

- Material Terms

- Other Terms

9. HANDLING EQUIPMENT

- Generic Terms

- Defined Terms

10. HEALTH PROTECTION

- Generic Terms

-. Defined Terms

- Assimilation/Exposure Terms

- Contamination Control Terms

- Monitoring Instrumentation/Alarm Terms

11. INSTRUMENTATION/CONTROL

- Generic Terms

- Defined Terms

- Analytical Terms

- Calibration Terms

- Control Activities Terms

- Distributive Control System (DCS) Terms

- Equipment Terms

12. PROCESS

Generic Terms

- Equipment Terms

- General Chemical Terms 
TABLE 8

\section{EQUIPMENT CODE (cont'd)}

(CATEGORY ORDER INDEX)

12. PROCESS (cont'd)

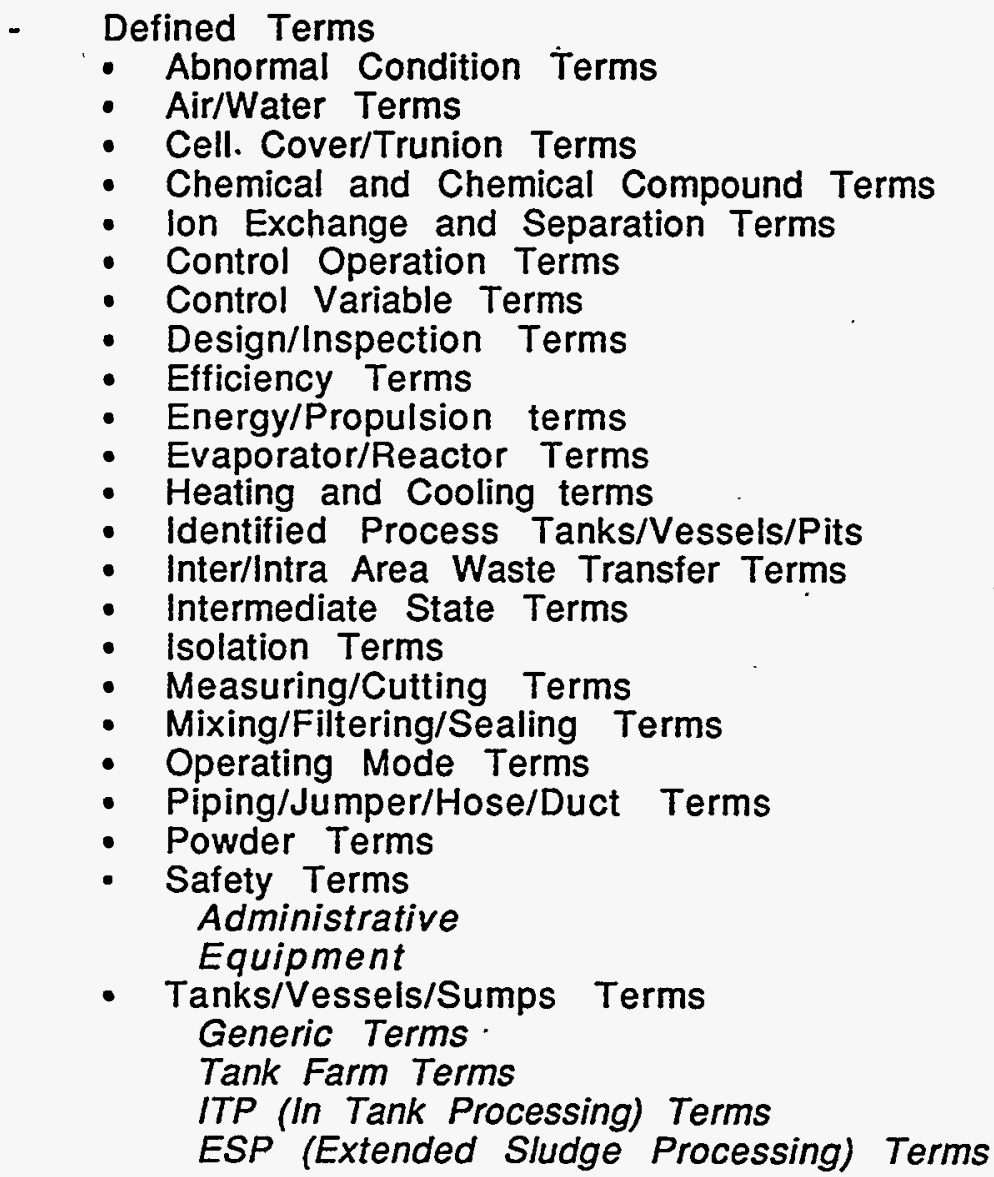

\section{QUALITY ASSURANCE}

14. SAFETY

- Personnel Safety

- Process Safety 
TABLE 8

EQUIPMENT CODE (cont'd)

(CATEGORY ORDER INDEX)

15. SECURITY

16. TRAINING

17. TRANSPORTATION

- Generic Terms

- Defined Terms

- Aircraft, Fuel, Roadways, Vehicles Terms

- Railroad Terms

- Consequence Terms

18. VENTILATION

- Generic Terms

- Defined Terms

19. VESSEL VENT

20. WASTE

- Generic Terms

- Defined Terms

- Composition terms

- Incineration/Compactor

21. WORKS ENGINEERING

- Generic Terms

- Defined Terms

- Work Package

- Design 
TABLE 9

EQUIPMENT CODE (CATEGORY ORDER NUMERICAL LISTING)

1. ADMINISTRATIVE

Plant Organization Terms

257 Reactor Division

514 Technical Division ( SRTC)

517 Stores, Salvage, Forestry, SREL,(ERDA),

558 Construction Division

Emergency Response/Criticality Terms

005 Criticality

041 Natural Phenomenon

097 NIM

285 Evacuation

439 Bell

Procedure Terms

030 Procedure Violation

054 Procedure Difficulty

395 Procedure Inadequacy

Human Factors Terms

073 Stress

298 Personnel Difficulty

Vendor Related Terms

084 Nooter

127 Vendor
671 Wackenhut Services

684 SREL, DOE, Subcontractor

905 SCDHEC

923 Bechtel 
TABLE 9

EQUIPMENT CODE (cont'd)

(CATEGORY ORDER NUMERICAL LISTING)

\section{CONTAINERS: SHIPPING AND STORAGE}

Generic Terms - General

$166 \quad \mathrm{Bag}$

186 Offsite

239 Shipping Container

243 Shipper, Responsibility

251 Shipping Papers

507 Primary Packaging

508 Secondary Packaging

Defined Terms

$\begin{array}{ll}117 & \text { Crucible } \\ 143 & \text { Vessel/Tank/Container } \\ 166 & \text { Bag } \\ 207 & \text { Cask } \\ 269 & \text { Storage Rack } \\ 416 & \text { Storage ( In Cell) } \\ 457 & \text { Dumpster } \\ 505 & \text { Cans, Drums }\end{array}$

\section{COMMUNICATION}

159 Communication - Phone

806 Communication Systems

\section{ELECTRICAL}

Generic Terms- General
512 Tie Down/Closure

620 Salvage Yard

\author{
511 Tank Trailer \\ 555 Vault \\ 742 Canister \\ 746 Container: B-25 \\ 750 B12 \\ 922 Special Engineered Box, \\ Sleeve, tube, etc. \\ 925 Skip Pan
}

025 Jumper

$438 \quad$ Battery 
TABLE 7

EQUIPMENT CODE (cont'd)

(CATEGORY ORDER NUMERICAL LISTING)

\section{ELECTRICAL (Cont'd)}

Generic Terms- General (Cont'd)

092 Electrical Distribution

201 Cables/Wiring

218 Short Circuit- Electrical

263 Lights

282 Alarm/Horn

286 Power Outage

428 Heater/Heating Element/

Steam Tracing

Defined Terms

- Emergency Power

$\begin{array}{lll}098 & \text { Emergency Power } \\ 470 & \text { Diesel }\end{array}$

- Equipment

066 Motor

131 Motor Generator

193 Switch

201 Cables/Wiring/Cords

222 Breaker

224 Electrical Contact

308 Transformer-Electrical

438 Battery

445 Meter

446 Regulator

447 Relay
444 Flasher

449 Trouble Light

475 Electrical Equipment Damaged

525 Loss of Load

939 Support Structures, Electrical Wire/

Steam Header

739 Uninterruptable Power Supply (UPS)

792 Standby

545 Interlock

625 Motor Control Center

761 Fuse

$767^{\circ}$ Field Terminal Cabinet/Junction Box

793 Power Supply 
TABLE 9

EQUIPMENT CODE (cont'd)

(CATEGORY ORDER NUMERICAL LISTING)

\section{ELECTRICAL (Cont'd)}

Defined Terms

- Equipment (cont'd)

$\begin{array}{llcll}448 & \text { Resistor } & \cdot & \\ 462 & \text { Insulation/Insulator } & 942 & \begin{array}{l}\text { Automatic } \\ \text { Switch }\end{array} & \text { Transfer } \\ & \text { - } & & \text { Capacitor } & \\ 492 & \text { Generator/Charger } & 943 & \text { Capacid } & \\ 501 & \text { Bearings } & 952 & \text { Solenoid } \\ & & 966 & \text { Circuit }\end{array}$

\section{ENVIRONMENTAL}

Generic Terms General

041 Natural Phenomenon

042 Ice, Frozen

536 Rain

047 Seepage Basin

550 Drill/Drilling

072 Lightning

631 Wind

145 Humidity

162 Underground

643 Storm Water

180 Retention Basin

727 Well Water

264 Water

728 Heat/Temperature

281 Oil Film

887 Seismic

290 Released

893 Tornado

983 Diversion/Flood/Sluice Gate/Valve

294 Surface Water

306 Delaying Basin

489 Wells

989 Vegetation/Algae

991 Erosion. 
TABLE 9

EQUIPMENT CODE (cont'd)

(CATEGORY ORDER NUMERICAL LISTING)

\section{ENVIRONMENTAL (Cont'd)}

Defined Terms

- Air/Effluent Monitor System Terms

279 Stack Monitor

559 Meteorological Instruments

636 Trebler Monitor

811 Process Contamination Monitors(Trebler, CAM, Storm Water etc.)

- Environmental Releases Terms

$\begin{array}{ll}046 & \text { Four Mile Creek } \\ 057 & \text { Sewer } \\ 095 & \text { Three Runs Creek } \\ 158 & \text { Stack } \\ 162 & \text { Underground } \\ 196 & \text { Outlet Weir } \\ 281 & \text { Oil Film }\end{array}$

290 Released

295 Ground Water

304 Diverted

619 Release Guides

644 Ditch

196 Outlet Weir

6. ERRORS/FAILURES/CAUSES/ROOT CAUSES

Generic Terms

$\begin{array}{llll}058 & \text { Design } & 255 & \text { Unauthorized } \\ 073 & \text { Stress } & 283 & \text { Ruptured } \\ 250 & \text { Errors } & 284 & \text { False }\end{array}$

Defined Terms (See 12, PROCESS, Abnormal Condition)

001 Transfer Error

002 Overflow 
TABLE 9

EQUIPMENT CODE (cont'd) (CATEGORY.ORDER NUMERICAL LISTING)

\section{ERRORS/FAILURES/CAUSES/ROOT CAUSES (Cont ${ }^{*}$ ) \\ Defined Terms (See 12, PROCESS, Abnormal Condition)}

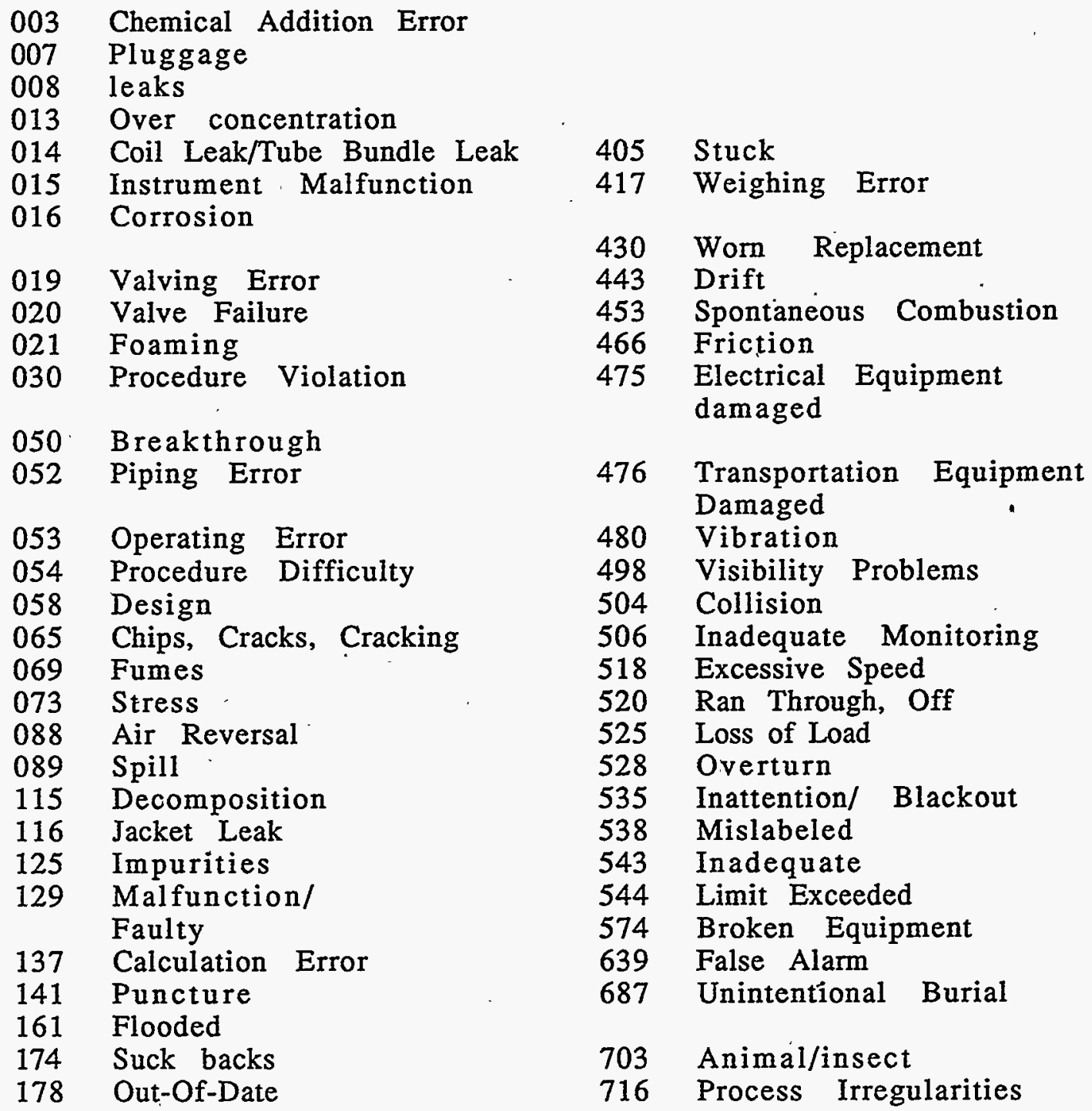


TABLE 9

EQUIPMENT CODE (cont'd) (CATEGORY ORDER NUMERICAL LISTING)

\section{ERRORS/FAILURES/CAUSES/ROOT CAUSES (Cont'd)}

Defined Terms (See 12. PROCESS, Abnormal Condition)

\begin{tabular}{|c|c|c|c|}
\hline 192 & Loss & 724 & Unauthorized container \\
\hline 202 & Alignment & 725 & Improper storage \\
\hline 205 & Dropped/Fell & 749 & Micro biologically \\
\hline 206 & Mechanical Failure & & Induced \\
\hline 212 & Overload & & Corrosion ( MIC) \\
\hline 214 & Hit Floor & & \\
\hline 215 & Hit Other Than Floor & & \\
\hline 217 & Jammed Component & & \\
\hline 218 & Short-Circuit Electrical & & \\
\hline 219 & Mechanical Damage & & \\
\hline 221 & Inadequate Function & & \\
\hline 225 & Faulty Component & & \\
\hline 229 & Protective Device Missing & 766 & Steam Collapse \\
\hline 231 & Broken Damaged & 803 & Control Program Error \\
\hline 235 & Supervisor Error & 809 & Lock and Tag Violation \\
\hline 236 & Incomplete Job & 822 & $\begin{array}{l}\text { Inadequate } \\
\text { Administrative Control }\end{array}$ \\
\hline 237 & Incorrect Position & 823 & Work Order Deficiency \\
\hline 249 & Improper Packaging & 872 & Personnel Error \\
\hline 250 & Errors & 880 & Conduct Of Operations \\
\hline 254 & Missing/Unavailable & 888 & Pipe Leak \\
\hline 255 & Unauthorized & & \\
\hline 259 & Locked Out & & \\
\hline 271 & Not Locked Out or tagged Out & 895 & $\begin{array}{l}\text { Design Error/Design } \\
\text { Improvement }\end{array}$ \\
\hline 283 & Ruptured & & \\
\hline $\begin{array}{l}284 \\
286\end{array}$ & $\begin{array}{l}\text { False } \\
\text { Power Outage }\end{array}$ & & \\
\hline 298 & Personnel Difficulty & & - \\
\hline 309 & Derail & & \\
\hline 39. & Procedure Inadequate & & \\
\hline 403 & Water Hammer & & \\
\hline
\end{tabular}


7. FIRE

Generic Terms-General

069 Fumes

076 Fire

109 Dust

282 Alarm

291 Cutting

412 Welding

453 Spontaneous Combustion

460 Sparks

467 Smoking Material

473 Misc. .Or Unknown Material Burned

Generic Terms-Combustible Types

$\begin{array}{ll}276 & \text { Waste } \\ 401 & \text { Coal } \\ 458 & \text { Paper } \\ 459 & \text { Cloth/Canvas } \\ 463 & \text { Grass } \\ 465 & \text { Solvent }\end{array}$

8. GENERIC TERMS-GENERAL

\section{ACTION TERMS}

\section{Loss}

212 Overload

238 Insecure

290 Released

304 Diverted
474 Misc. or Unknown Ignition Source

639 False Alarm

646 Fire Detection \& Suppression

673 Halon

762 Smoke

468 Gasoline

469 Wood

471 Metal

472 Gas

692 Hydraulic Fluid

$\begin{array}{ll}192 & \text { Loss } \\ 212 & \text { Overload } \\ & \\ 238 & \text { Insecure } \\ 290 & \text { Released } \\ 304 & \text { Diverted }\end{array}$

432 Repair

504 Collision.

512 Tie Down, Closure

552 Install, Replace and/or Remove

792 Standby 
TABLE 9

EQUIPMENT CODE (cont'd) (CATEGORY ORDER NUMERICAL LISTING)

\section{GENERIC TERMS-GENERAL}

EOUIPMENT TERMS

128 Equipment

226 Protective Device Added

227 Brake

MATERIAL TERMS

.462 Insulation/Insulator

469 Wood

471 Metal

QTHER TERMS

130 Accountability

133 Caustic Alkali

154 Personnel

162 Underground

178 Out of Date

186 Off-Site
229 Protective Device Missing

455 Gear Box /Gears

477 Refrigeration

501 Bearing,

626 Door

640 Encasement/Jacket

658 Tool

472 Gas

633 Concrete

431 Missiles

542 Room

543 Inadequate

620 Salvage Yard

692 Hydraulic Fluid

694 Burner

699 Less Than 10 Gallons

700 Greater Than 10 Gallons 
TABLE 9

EQUIPMENT CODE (cont'd)

(CATEGORY ORDER NUMERICAL LISTING)

\section{HANDLING EQUIPMENT}

\section{GENERIC TERMS}

$227 \quad$ Brake

DEFINED TERMS

059 Drop Line

086 Screw Conveyor

107 Elevator/Dumbwaiter

118 Hydraulic. Systems

181 Lifting Bail

200 Cranes, Hoists, Traveling Bridge Turntables

201 Cables/Wires/Cords

208 Monorail

211 Yoke/Grapple

213 Hook

\section{HEALTH PROTECTION}

\section{GENERIC TERMS}

037 Activity

048 Contamination: Facility or Equipment

055 RCA
300 Impact Wrench

692 Hydraulic Fluid

404 Bucket/Clamshell

406 Coal Handling Equipment

512 Closure. Tie Down

529 Fork lift/Motor Crane, Tractor/Lift Truck

627 Manipulator

648 Cart

691 Lazy Susan

768 Center Load

790 Conveyor

801 Pneumatic Conveyor

807 Incell Crane

812 Transporter

908 Transfer Car
153 Contamination Airborne

415 Decontamination

548 Skin Contamination

549 Monitoring 
TABLE 9

EQUIPMENT CODE (cont'd) (CATEGORY ORDER NUMERICAL LISTING)

\section{HEALTH PROTECTION (Cont'd)}

\section{GENERIC TERMS ·}

$\begin{array}{ll}093 & \text { Blowers, Fans } \\ 106 & \text { Radiation } \\ 114 & \text { Exhausters } \\ 120 & \text { Sampling }\end{array}$

751 Contamination

752 Radioactive

875 Fixed Contamination

876 Smearable Contamination

937 Industrial Hygiene

993 Detector

DEFINED TERMS

- ASSIMILATION-EXPOSURE TERMS

056 Personal Exposure 479 Uptake/ Injection 153 Contamination- Airborne 548 Skin Contamination 155 Uptake/ Ingestion 582

156 Inhalation 682 Nasal Contamination

478 Uptake/ Inhalation 683 Uptake/ Absorption

- CONTAMINATION CONTROL TERMS

$\begin{array}{llrl}050 & \text { Breakthrough } & 429 & \text { Glove Change } \\ 079 & \text { Protective Clothing } & 672 & \text { Clothing Contamination } \\ 111 & \text { Gloves } & 877 & \text { Personal Clothing } \\ 150 & \text { Hut } & 924 & \text { Shielding }\end{array}$

- MONITORING INSTRUMENTATION/ALARM TERMS

$158 \quad$ Stack

270 H.M. Alarm

804 Personnel Contamination Monitor 
TABLE 9

EQUIPMENT CODE (cont'd) (CATEGORY ORDER NUMERICAL LISTING)

\section{HEALTH PROTECTION (Cont'd)}

- MONITORING INSTRUMENTATION/ALARM TERMS

$\begin{array}{llrl}279 & \text { Stack Monitor } & 805 & \begin{array}{l}\text { Personnel Radiation } \\ \text { monitor }\end{array} \\ 441 & \begin{array}{l}\text { Chamber } \\ 450\end{array} & \begin{array}{l}\text { Neutron Monitoring } \\ \text { Process Radiation Monitor }\end{array} \\ 506 & \begin{array}{l}\text { Inadequate Monitoring } \\ \text { M59 }\end{array} & 811 & \begin{array}{l}\text { Process Contamination } \\ \text { Monitorological }\end{array} \\ & \begin{array}{l}\text { Instrumentation } \\ \text { Trebler Monitor }\end{array} & 824 & \begin{array}{l}\text { Source Check } \\ \text { Detector }\end{array} \\ & & 993 & \end{array}$

11. INSTRUMENTATION/CONTROL

GENERIC TERMS

263 Lights

282 Alarm

428 Heater/ Heating Element/

Stream Tracing

438 Battery

441 Chamber

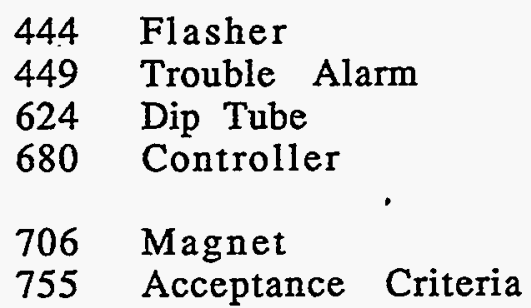

DEFINED TERMS

- ANALYTICAL TERMS (See 12. PROCESS - Sampling)

120 Sampling

124 Analytical

125. Impurities

427 Mass Spectrometer
523 Samples

628 Coil Pressure System

635 PHA

698 Oxygen Analyzer

753 Hydrogen Analyzer 


\section{INSTRUMENTATION/ CONTROL (Cont'd)}

DEFINED TERMS

- ANALYTICAL TERMS ( See 12. PROCESS - Sampling)

491 Balance

- CALIBRATION TERMS

440 Calibration

- CONTROL ACTIVITIES TERMS

\section{Temperature Control}

096 Flow Control

440 Calibration
754 Lower Explosive Limit Analyzer

882 Benzene Analyzer

902 Minimum Oxygen For Combustion (MOC)

994 Lysimeter

443 Drift

500 Liquid Level

759 Pressure Control

- DISTRIBUTIVE CONTROL SYSTEM (DCS)/COMPUTER TERMS

$\begin{array}{llrl}481 & \text { Computer } & 871 & \text { Cards/Modules/Boards } \\ 675 & \text { Robot } & & \\ 697 & \text { Combustion Programmers } 873 & \text { Programmable Logic } \\ 803 & \text { Computer Program Error } & \text { Control } \\ & & 899 & \begin{array}{l}\text { Distributed Control System } \\ \text { Printer/Scanner }\end{array}\end{array}$

- EQUIPMENT TERMS

026 Thermohm

074 Thermocouple

123 Thermometers

545 Interlock

624 Dip Tube 
TABLE 9

EQUIPMENT CODE (cont'd) (CATEGORY ORDER NUMERICAL LISTING)

11. INSTRUMENTATION/CONTROL (Cont'd) DEFINED TERMS

- EQUIPMENT TERMS

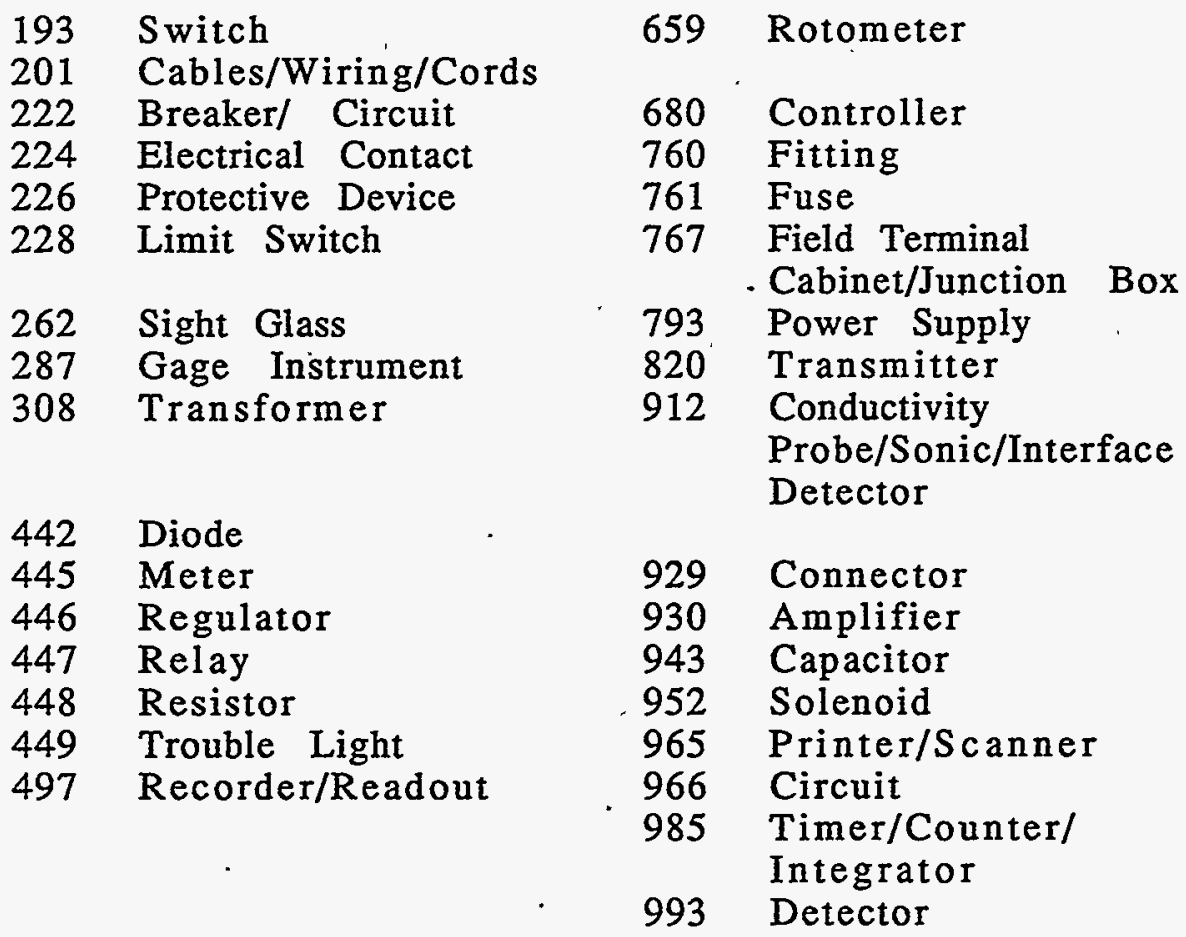

\section{PROCESS:}

\section{GENERIC TERMS}

- Equipment

$\begin{array}{llll}024 & \text { Gasket/Flange } & 790 & \text { Conveyor } \\ 049 & \text { Acid Absorber } & 794 & \text { Decanter }\end{array}$.


TABLE 9

EQUIPMENT CODE (cont'd) (CATEGORY ORDER NUMERICAL LISTING)

\section{PROCESS: (Cont'd)}

\section{GENERICTERMS}

- Equipment

$\begin{array}{ll}066 & \text { Motor } \\ 110 & \text { Compressor } \\ 118 & \text { Hydraulic System } \\ 136 & \text { Header } \\ & \\ 182 & \text { Decon Cell } \\ 277 & \text { Waste Cell } \\ 305 & \text { Cooling Tower } \\ 477 & \text { Refrigeration } \\ 493 & \text { Precipitator } \\ 501 & \text { Bearings } \\ 676 & \text { Funnel/Hopper } \\ 681 & \text { Dryer } \\ 770 & \text { Collection tank }\end{array}$

- Facilities

\begin{tabular}{|c|c|c|c|}
\hline 825 & Cold Feed Prep & 851 & Process Cell \\
\hline 844 & Chemical Process Cell & 860 & 299-H \\
\hline 845 & Salt Process Cell & 869 & $\begin{array}{l}\text { ITP Filter Building 241- } \\
96 \mathrm{H}\end{array}$ \\
\hline $\begin{array}{l}846 \\
847\end{array}$ & $\begin{array}{l}\text { Canister Cell } \\
\text { Contact Decon and } \\
\text { Maintenance Cell }\end{array}$ & 906 & Pump House \\
\hline $\begin{array}{l}848 \\
849\end{array}$ & $\begin{array}{l}\text { Weld Cell } \\
\text { Sample and Analytical } \\
\text { Cell }\end{array}$ & $\begin{array}{l}992 \\
999\end{array}$ & $\begin{array}{l}\text { Barricade/Fence/ } \\
\text { Enclosure/Dike } \\
\text { RBOF. }\end{array}$ \\
\hline 850 & Melter Cell & & \\
\hline
\end{tabular}

826 Transfer tank

827 Receiving tank

828 Storage tank

855 Condensate Tank

856 Feed Tank

881 Surge Tank

883 Waste Tank

892 Pump Tank

935 Process Chiller

951 Seals/Packing/Caulking

996 Diaphram 
TABLE 9

EQUIPMENT CODE (cont'd) (CATEGORY ORDER NUMERICAL LISTING)

\section{PROCESS: (Cont'd)}

\section{GENERIC TERMS}

- General Chemical

$\begin{array}{llll}039 & \text { Organic } & 465 & \text { Solvent } \\ 064 & \text { Teflon } & 494 & \text { Coating (lining)/Liner } \\ 133 & \text { Caustic/Alkali } & 799 & \text { Refractory } \\ & & 800 & \text { Plug }\end{array}$

\section{DEFINED TERMS}

- Abnormal Condition Terms

$\begin{array}{ll}002 & \text { Overflow } \\ 003 & \text { Chemical Addition Error } \\ 004 & \text { Uncontrolled Reaction } \\ & \\ 006 & \text { Siphoning } \\ 007 & \text { Pluggage } \\ & \\ 008 & \text { Leaks } \\ 009 & \text { Eructation } \\ 010 & \text { Boilover } \\ 011 & \text { Boiloff } \\ 012 & \text { Explosion } \\ 013 & \text { Over Concentration } \\ 014 & \text { Coil Leak } \\ 015 & \text { Instrument Malfunction } \\ 021 & \text { Foaming } \\ 042 & \text { Ice/Frozen } \\ 043 & \text { Column Flooding } \\ 050 & \text { Breakthrough } \\ 053 & \text { Operating Error } \\ 054 & \text { Procedure Difficulty }\end{array}$

204 Snatched

205 Dropped/fell

214 Hit Floor

215 Hit Other Than Floor

217 Jammed Component

219 Mechanical Damage'

223 Unknown Material

231 Broken, Damaged

237 Incorrect Position

245 Came Apart

254 Missing

258 Material Lost

282 Alarm/Horn

304 Diverted

309 Derail

430 Worn Replacement

431 Missile

449 Trouble Light

475 Electric Equipment

Damaged

480 Vibration 
TABLE 9

EQUIPMENT CODE (cont'd) (CATEGORY ORDER NUMERICAL LISTING)

\section{PROCESS: (Cont'd)}

\section{DEFINED TERMS}

- Abnormal Condition Terms (cont'd)

054 Procedure Difficulty

065 Chipped, Cracks Cracking

069 Fumes

089 Spill

112 Deformation

125 Impurities

129 Malfunction/Faulty

161 Flooded

- Air/Water Terms

Air

110 Compressor

151 Breathing Air

477 Refrigeration

482 Compressed Air

496 Instrument Air

Water

036 Process Water
498 Visibility Problem

504 Collision

506 Inadequate Monitoring

520 Ran Through, Off

538 Mislabeled

639 .False Alarm

701 Ash Buildup

716 Process Irregularities

884 Deflagration

887 Seismic

888 Pipe Leak
622 Process Air

681 Air Dryer

705 Plant Air
303 Segregated Water

305 Cooling Tower

477 Refrigeration

489 Wells 
TABLE 9

EQUIPMENT CODE (cont'd)

(CATEGORY ORDER NUMERICAL LISTING)

\section{PROCESS: (Cont'd)}

\section{DEFINED TERMS}

- Air/Water Terms (cont'd)

Water

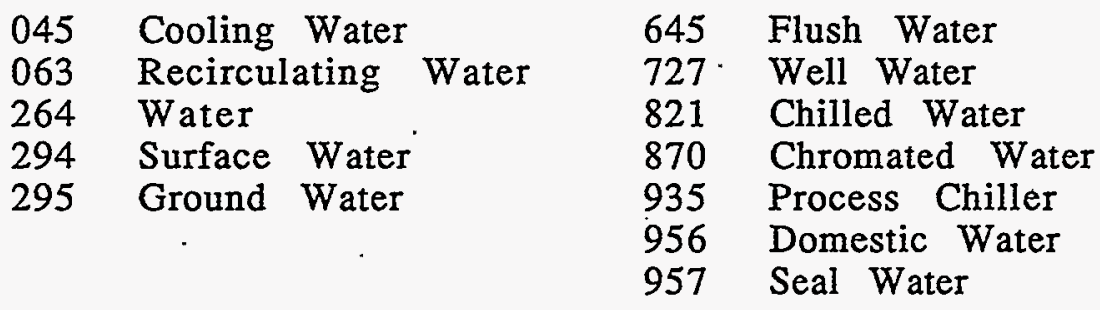

- Cell Covers/Trunions

\section{Cell Covers}

203 Trunions

- Chemicals and Compounds Terms
038 Uranium
044 Nitric Acid
630 Ruthenium
642 Sodium
668 Helium
051 Silica Gel
690 Hazardous Chemicals
101 Inert Gas
709 Mercury
103 Plutonium
758 Nitrogen
104 Neptunium
785 Benzene
133 Caustic/Alkali
786 Formic Acid
152 Resin
787 Oxalic Acid
172 HAN
788 Sodium Hydroxide
188 Catalyst
798 Frit
194 Iodine
198 Hydrogen
452 Chlorides
502 Ammonia Compounds
897 Sodium Nitrite
898 Sodium Nitrate
890 Sodium Tetraphẹnyborate 
TABLE 9

EQUIPMENT CODE (cont'd) (CATEGORY ORDER NUMERICAL LISTING)

\section{PROCESS: (Cont'd)}

\section{DEFINED TERMS}

- Chemicals and Compounds Terms (cont'd)

503 Floride Compounds

901 Formic Acid

527 Tritium, D2O

904 Carbon Dioxide

537 Thorium

554 Americium

- Control Operations Terms

$\begin{array}{ll}100 & \text { Purging } \\ 126 & \text { Drain } \\ 135 & \text { Sparge } \\ 141 & \text { Puncture } \\ 149 & \text { Valence Adjustment } \\ 164 & \text { Decant }\end{array}$

$\begin{array}{ll}304 & \text { Diverted } \\ 410 & \text { Melting/Pouring } \\ 414 & \text { Cabinet Entry/Exit of } \\ & \text { Material } \\ 433 & \text { Leak Check } \\ 512 & \text { Tie Down/Closure } \\ 552 & \text { Install/Replace and/or } \\ & \text { Remove } \\ 553 & \text { Test } \\ 701 & \text { Spray Quench/Spray/ } \\ & \text { Spray Chamber }\end{array}$

- Control Room/Shops Terms

179

$$
\begin{aligned}
& \text { Control Room/ } \\
& \text { Control Board/ } \\
& \text { Panel Control }
\end{aligned}
$$

- Control Variables Terms

\section{Temperature Control \\ 070 Vacuum}

183 Shop

541 Maintenance Room

542 Room

626 Door 
TABLE 9

EQUIPMENT CODE (cont'd) (CATEGORY ORDER NUMERICAL LISTING)

12. PROCESS: (Cont'd)

DEFINED TERMS

- Control Variables Terms (cont'd)

096 Flow Control

144 Pressure

145 Humidity

280 Turbidity 728

456 Process Heat 729

480 Vibration . 759

Heat/Temperature

Flow

Pressure Control

Specific Gravity

- Effluent Treatment Facility

911 Effluent Treatment Facility

- Design/Inspections

058 Design

233 Structural Component

252 Dimensions

433 Leak Check

- Efficiency Terms

289 Inventory

- Energy/Propulsion

$\begin{array}{ll}060 & \text { Steam } \\ 066 & \text { Motor }\end{array}$

551

553

717

718

889

895

665

Downtime/Repair Time

170

456

Inspection

Test

Inspections Annulus

Inspection inside Tank

Hydrotest

Design Error/Design

Improvement

665 Downtime/Repair Time

$$
\begin{aligned}
& \text { Decay Heat } \\
& \text { Process Heat }
\end{aligned}
$$


12. PROCESS: (Cont'd)

\section{DEFINED TERMS}

- Energy/Propulsion (cont'd)

070 Vacuum

466

728

Friction

Heat/Temperature

- Evaporator/Reactor Terms

034 Condenser

163 Evaporator

713 Transfer Tank to Evaporator

797

714 Transfer: Evaporator to Tank

715 Transfer: Evaporator to Condensate

732 Evaporator 1

733 Evaporator 2

- Heating and Cooling Terms

060 Steam

102 Furnace

132 Heating Coil/Vaporizer

167 Cooling Coil

187 Heat Exchanger/Cooler

232

402

422

425

428
Bundle/Tube Bundle

Boiler

Furnace, Vacuum

Furnace, Induction

Heater, Heating Element
477

632

728

764

941

988
772

796

Slurry Mix Evaporator

Precipitate Reactor

Organic Evaporator

Condensate System

Evaporator Overhead-

Evaporator Bottom

Evaporator Bottom

Pump Tank

954 Desalt/Descale
Refrigeration

Dehumidifier

Heat/Temperature

Process Chillers

Condensate System

Air Condition 
TABLE 9

EQUIPMENT CODE (cont'd)

(CATEGORY ORDER NUMERICAL LISTING)

\section{PROCESS: (Cont'd)}

\section{DEFINED TERMS}

- Identified - Process Tanks/Vessels/Pits/Facilities

390

732

733

740

741

757

770

772

773

774

775

776

777

778

779

780

781

782

783

784

796

797

802

825

826

827
Pump Pits

Evaporator 1

Evaporator 2

Sludge Receipt and Adjustment Tank

Glass Melter

Building 641-G 1AL

Pump Pit

Collection tank

Slurry Mix Evaporato

Low Point Pump Pit 511-S

Service Building 210-S

Glass Waste Storage

Building 250S

Exhaust Stack 291-S

Fan House 292-S

Sand Filter 294-S

Bulk Frit \& Chemical

Storage 422-S

Organic Waste Storage

Facility 430-S

Cooling Tower 981-S

Primary Substation 951-S DCS Staging Building 706-S

Water \& Chemical Waste 960 Treatment Building 980S 961

Precipitate Reactor 962

Organic Evaporator 963

Aux. Pump Pit(512-S) 979

Cold Feed Prep

Transfer Tank

982

984

986
Surge Tank

Waste Tank

Cesium Removal Column

Pump. Tank

Vitrification Building 221-S

906 Pump House

938 Recycle Collection Tank

940 Decontamination Waste Treatment tank

958 Hot Decontamination Waste Header

959 Warm Decontamination Waste Header

Formic Acid Waste Header Mercury Transfer Header Floor Drain Catch Tank Acid Drain Catch Tank Catch Tánk Building 210-Z Valve pit/valve box Late Wash Facility 
TABLE 9

EQUIPMENT CODE (cont'd) (CATEGORY ORDER NUMERICAL LISTING)

\section{PROCESS: (Cont'd)}

\section{DEFINED TERMS}

- Identified Process Tanks/Vessels/Pits/Facilities (cont'd)

828 Storage Tank

855 Condensate Tank

856 Feed Tank

860 299-H

- Inter/Intra Area Waste Transfer Terms

\begin{tabular}{|c|c|c|c|}
\hline 136 & Header & 931 & $\begin{array}{l}\text { Lead Detection, } \\
\text { Box/Sump/Pot }\end{array}$ \\
\hline 89 & Diversion Box & 756 & Bldg. 511-S Pump Pit \\
\hline & Pump Pit & 757 & Bldg. 641-G IAL Pump Pit \\
\hline 18 & Transfer Locks & 773 & Low Point Pump Pit \\
\hline 3 & Leak Check & & \\
\hline y & Coating/lining & 802 & Auxiliary Pump Pit(Late \\
\hline 47 & Transfer System & & Wash Facility) \\
\hline 40 & Encasement/Jacket & 906 & Pump House \\
\hline 545 & Flush Water & 920 & Gang Valve House \\
\hline 6 & $\begin{array}{l}\text { Transfer Device } \\
\text { Transfer } 221 \text { to } 241\end{array}$ & 931 & $\begin{array}{l}\text { Lead Detection, Box/Sump } \\
\text { /Pot }\end{array}$ \\
\hline & Transfer within 241 & 947 & $\begin{array}{l}\text { Evaporator Bottom Pump } \\
\text { Tank }\end{array}$ \\
\hline 12 & $\begin{array}{l}\text { Transfers: Concentrate } \\
\text { Transfer System }\end{array}$ & 948 & CTS Loop Line \\
\hline 1 & $\begin{array}{l}\text { Concentrate Transfer } \\
\text { System ( CTS ) }\end{array}$ & 953 & $\begin{array}{l}\text { Transfers: Inter Area } \\
\text { Transfer Line }\end{array}$ \\
\hline
\end{tabular}

- Intermediate State Terms

018 Precipitation

814 Precipitate

169 Emulsification 
TABLE 9

EQUIPMENT CODE (cont'd) (CATEGORY ORDER NUMERICAL LISTING)

\section{PROCESS: (Cont'd)}

\section{DEFINED TERMS}

- Intermediate State Terms (cont'd)

256 Sediments

266 Saturation

280 Turbidity

521 Debris

808 Salt Formation
$816 \quad$ Sludge

817 Salt

818 Glass

819 Recycle

896 Supernate

- Ion Exchange and Separation Terms

105 Elution

119 Deionizer/

Ion Exchange

Columns

149 Valence Adjustment

152 Resin

164 Decant

173 Separator

- Isolation Terms

$\begin{array}{ll}138 & \text { Blank } \\ 157 & \text { Gang Valve } \\ 165 & \text { Pipe } \\ 196 & \text { Outlet/Weir } \\ 227 & \text { Brake }\end{array}$

- Measuring/Cutting

291 Cutting

435 Reel Tape
188

493

634

743

795

813

886

981

389

546

623

640

931

738

932
Catalyst

Precipitator

Scrubber

Mercury Purification

Column

Stripping Column

Reverse Osmosis

Cesium Removal Column

Catalyst

\section{Diversion Box}

Trap

Damper

Encasement/Jacket

Leak Detection, Box/

Sump/Pot

Pipe Cutter

Steel Tape 
TABLE 9

EQUIPMENT CODE (cont'd) (CATEGORY ORDER NUMERICAL LISTING)

12. PROCESS: (Cont'd)

DEFINED TERMS

- Mixing/Filtering/Sealing Terms

Filtering 090 Filter (General)/Screen 813 Reverse Osmosis
Strainer

Mixing

068 Agitator

184 Mixer-Settler Bank

539 Vibrator

299 Washer

Sealing Equipment

075 Weld

176 Seal Pot

412 Welding

789 Welder

$800 \quad$ Plug

- Operating Modes Terms

146 Shutdown

730 Startup

765 Switchover

- Piping/Jumper/Hose/Duct/Belt

Generic Terms

$\begin{array}{lllll}024 & \text { Flange/Gasket } & 261 & \text { hose } & \\ 025 & \text { Jumper } & 490 & \text { Duct } & \\ 136 & \text { Header } & & & \\ 147 & \text { Embedded Pipe } & & & \\ 148 & \text { Rack Pipe } & 771 & \text { Temporary Piping }\end{array}$


EQUIPMENT CODE (cont'd)

(CATEGORY ORDER NUMERICAL LISTING)

\section{PROCESS: (Cont'd)}

\section{DEFINED TERMS}

- Piping/Jumper/Hose/Duct/Belt

Generic Terms (cont'd)

$\begin{array}{llll}165 & \text { Pipe } & 862 & \text { Expansion Joint } \\ 168 & \text { Nozzle } & 929 . & \text { Connectors } \\ 175 & \text { Steam Trap } & 980 & \text { Belt }\end{array}$

- Powder Terms

$\begin{array}{llll}080 & \text { Collection Dust } & 109 & \text { Dust } \\ 082 & \text { Dust Bag } & 426 & \text { Powder } \\ 085 & \text { Pulverizer } & 736 & \text { Premix-Blending } \\ 086 & \text { Screw Conveyor } & 737 & \text { Salt-Cement Mixing } \\ & & 790 & \text { Conveyor } \\ & & 801 & \text { Pneumatic Conveyor }\end{array}$

- Safety Terms

Administrative

142 Technical Standards/ Operating Limits

240 Responsibility SR00

241 Responsibility SRS

260 Closed Vessel Entry

619 Release Guides

731 Operation Safety Requirement (OSR)

734 OSR Violation

748. Permit Violation

755 Acceptance Requirements

809 Lock and Tag Violation 
TABLE 9

EQUIPMENT CODE (cont'd) (CATEGORY ORDER NUMERICAL LISTING)

\section{PROCESS: (Cont'd)}

\section{DEFINED TERMS}

- Safety Terms
Administrative
823 Work Order Deficiency
880 Conduct of Operations
885 Unreviewed Safety Question
967 Process Hazard
889 Hydrotest
995 Safety Analysis Report (SAR)

- Safety Terms

Equipment

649 Rupture Disk

- Sampling (See 11. INSTRUMENT CONTROL • Analytical)

$\begin{array}{ll}120 & \text { Sampling } \\ 124 & \text { Analytical } \\ 125 & \text { Impurities } \\ 302 & \text { Door Stop }\end{array}$

- Structural

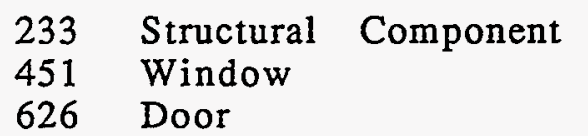

523 Samples

791 Pneumatic Transfer

System

\author{
852 Roof \\ 853 Floor \\ 854 Wall \\ 862 Expansion Joints
}


TABLE 9

EQUIPMENT CODE (cont'd) (CATEGORY ORDER NUMERICAL LISTING)

\section{PROCESS: (Cont'd)}

DEFINED TERMS

- Surveillance/test

$$
\begin{array}{ll}
301 & \text { Optics } \\
433 & \text { Leak Check } \\
451 & \text { Window } \\
549 & \text { Monitoring }
\end{array}
$$

- Tank Farm Basins

047 Seepage Basin

180 Retention Basin

- Tanks/Vessels/Sumps Terms

GENERIC TERMS

032 Sump

TANK FARM TERMS

$\begin{array}{ll}311 & \text { Tank } 1 \\ 312 & \text { Tank } 2 \\ 313 & \text { Tank } 3 \\ 314 & \text { Tank } 4 \\ 315 & \text { Tank } 5 \\ 316 & \text { Tank } 6 \\ 317 & \text { Tank } 7 \\ 318 & \text { Tank } 8 \\ 319 & \text { Tank } 9 \\ 320 & \text { Tank 10 } \\ 321 & \text { Tank 11 } \\ 322 & \text { Tank } 12\end{array}$

551 Inspection

553 Test

717 Inspection Annulus

718 Inspection Inside tank

720 TV Camera
306 Delaying Basin

948 Fuel/Target Storage Basin
143 Vessel, Tank, Container

$\begin{array}{ll}341 & \text { Tank } 31 \\ 342 & \text { Tank 32 } \\ 343 & \text { Tank 33 } \\ 344 & \text { Tank 34 } \\ 345 & \text { Tank } 35 \\ 346 & \text { Tank } 36 \\ 347 & \text { Tank } 37 \\ 348 & \text { Tank 38 } \\ \text { 349 } & \text { Tank 39 } \\ 350 & \text { Tank 40 } \\ \text { 351 } & \text { Tank 41 } \\ \text { 352 } & \text { Tank 42 }\end{array}$




\section{PROCESS: (Cont'd)}

\section{DEFINED TERMS}

TANK FARM TERMS (cont'd)

$\begin{array}{lll}323 & \text { Tank } 13 \\ 324 & \text { Tank } 14 \\ 325 & \text { Tank } 15 \\ 326 & \text { Tank } 16 \\ 327 & \text { Tank } 17 \\ 328 & \text { Tank } 18 \\ 329 & \text { Tank } 19 \\ 330 & \text { Tank 20 } \\ 331 & \text { Tank 21 } \\ 332 & \text { Tank 22 } \\ 333 & \text { Tank 23 } \\ 334 & \text { Tank 24 } \\ & \\ 335 & \text { tank 25 } \\ 336 & \text { tank 26 } \\ 337 & \text { Tank 27 } \\ 338 & \text { Tank 28 } \\ 339 & \text { Tank 29 } \\ 340 & \text { Tank } 30\end{array}$

353 Tank 43

354. Tank 44

355 Tank 45

356 Tank 46

357 Tank 47

358 Tank 48

359 Tank 49

360 . Tank 50

361 Tank 51

362 Tank 52

386 Tank Ventilation

387 Tank Annulus

Ventilation

435 Reel Tape

.346 Annulus

556 Riser

717 Inspection Annulus

718 Inspection Inside Tank

732 Tank Farm Evaporator 1

733 Tank Farm Evaporator 2

808 Salt Formation

ITP (In Tank Processing) TERMS

090 Filter(General)

351 Tank 41

358 Tank 48

758 Nitrogen

359 Tank 49

785 Benzene

795 Stripping Column

869 ITP Filter Building 
TABLE 9

EQUIPMENT CODE (cont'd) (CATEGORY ORDER NUMERICAL LISTING )

\section{PROCESS: (Cont'd)}

\section{DEFINED TERMS}

- Tanks/Vessels/Sumps Terms

ESP (Extended Sludge Processing) TERMS

332 Tank 22

361 Tank 51

350 Tank 40

352 Tank 42

- Transfer Terms (See 12. PROCESS - Inter/Intra Area: Waste Transfer Terms)

$\begin{array}{ll}.017 & \text { Jet } \\ 091 & \text { Pump } \\ & \\ 157 & \text { Gang Valve } \\ 265 & \text { Pump Seals } \\ 390 & \text { Pump Pits } \\ 547 & \text { Transfer System }\end{array}$

13. QUALITY ASSURANCE

723 Quality Control

14. SAFETY

Personal Safety

056 Personal Exposure

077 Injury

141 Puncture

216 Potential Major Injury
669 Transfer Device

791 Pneumatic Transfer

System

815 Slurry Pump

933 Feed Pump

934 Transfer Pump

955 Recirculation/Drum Off/Side

978 Impeller 
TABLE 9

EQUIPMENT CODE (cont'd)

(CATEGORY ORDER NUMERICAL LISTING)

14. SAFETY (Cont'd)

Personal Safety (cont'd)

$532 \quad$ Fatalities

708 Safety Shower

981 Safety Equipment

992 Barricade/Fence/

Enclosure

Process Safety (.See Process-Safety)

15. SECURITY

660 Safeguards

671 Wackenhut Services

747 Security

16. TRAINING

679 Training

17. TRANSPORTATION

Generic Terms

$\begin{array}{llll}141 & \text { Puncture } & & \\ 186 & \text { Off-Site } & 501 & \text { Bearing/Wheels } \\ 261 & \text { Hose } & & \\ 438 & \begin{array}{l}\text { Battery } \\ \text { Transportation } \\ \text { equipment damaged }\end{array} & 513 & \text { Chocks } . \\ & & \end{array}$


TABLE 9

EQUIPMENT CODE (cont'd) (CATEGORY ORDER NUMERICAL LISTING)

\section{TRANSPORTATION 、(Cont'd)}

\section{Defined Terms}

- Aircraft, Fuel, Roadways, Vehicles Terms

\begin{tabular}{|c|c|c|c|}
\hline $\begin{array}{l}066 \\
118\end{array}$ & $\begin{array}{l}\text { Motor } \\
\text { Hydraulic Systems }\end{array}$ & & \\
\hline 227 & Brake & 524 & Car, Truck \\
\hline 230 & Fuel & 529 & Fork Lift, Mc \\
\hline $\begin{array}{l}248 \\
438\end{array}$ & $\begin{array}{l}\text { Trailer } \\
\text { Battery }\end{array}$ & & Tractor \\
\hline 468 & Gasoline & $\begin{array}{l}533 \\
648\end{array}$ & $\begin{array}{l}\text { Pulpwood tr } \\
\text { Cart }\end{array}$ \\
\hline 470 & Diesel & 685 & Highway 125 \\
\hline 483 & Pavement/Asphalt & 686 & Aircraft \\
\hline 504 & Collision & 692 & Hydraulic Flu \\
\hline 509 & Roadway & 812 & Transporter \\
\hline 511 & Tank Trailer & 928 & Oil \\
\hline roac & Terms & & \\
\hline 210 & Rail Car/Cask Car/ & & \\
\hline & Flat Car & 309 & Derail \\
\hline 242 & Railroad Responsibility & $\begin{array}{l}510 \\
519\end{array}$ & $\begin{array}{l}\text { Road Bed (RF } \\
\text { Locomotive }\end{array}$ \\
\hline 296 & Tunnel & 522 & RR Crossing \\
\hline 297 & Railroad & 629 & Rail/ Track \\
\hline
\end{tabular}

- Consequence

$530 \cdot$ Jackknife

531 Skid 
EQUIPMENT CODE (cont'd) (CATEGORY ORDER NUMERICAL LISTING)

\section{VENTILATION}

Generic Terms

$\begin{array}{ll}050 & \text { Breakthrough } \\ 082 & \text { Dust Bag } \\ 477 & \text { Refrigeration }\end{array}$

Defined Terms

$\begin{array}{lll}023 & \text { Demister } \\ 087 & \text { Air Lock } & \\ 088 & \text { Air Reversal } & \\ 090 & \text { Filters(General, } & \text { Screens } \\ & \text { Strainers) } & \\ 093 & \text { Blowers, Fans } \\ 100 & \text { Purging } \\ 114 & \text { Exhauster } \\ 145 & \text { Humidity } \\ 158 & \text { Stack } \\ 166 & \text { Bag } \\ 199 & \text { Sand filter }\end{array}$

19. VESSEL VENT

023 Demister

034 Condenser

069 Fumes

070 Vacuum

071 Offgas

093 Blowers, Fans

292 Ventilation

386 Ventilation Tank

387 Ventilation Annulus

114 Exhausters

173 Separator

176 Seal Pot

386 Ventilation tank

623 Damper

634 Scrubber

638 HEPA Filter

764 Process Chiller

910 Vessel Vent/Vent 
TABLE 9

EQUIPMENT CODE (cont'd) (CATEGORY ORDER NUMERICAL LISTING)

\section{WASTE/RESTORATION}

Generic Terms

$186^{\circ}$ Off Site

195 Slugs

197 Transplutonium

234 Burial Ground

276 Waste

909 Hazardous Waste

913 Mixed Waste

914 TRU Waste

915 Waste-Process(Pipe, Valves, Jumper, Jets)

916 Waste-ElectricalInstrumentation Equipment

917 Waste-Job Control

918 Waste-Excavation ( Dirt, Asphalt, etc.)

690 Hazardous Chemicals

919 Waste-Ventilation Equipment (Filters, etc.)

829 Low Level Waste

830 Sanitary waste

834 Intermediate Level Waste
926 Scrap Process Metal

927 Hazardous Metals ( $\mathrm{CD}, \mathrm{Pb}$, $\mathrm{Hg}, \mathrm{Ag}$ )

992 Barricade/Fence/ Enclosure

- Composition

$\begin{array}{ll}039 & \text { Organic } \\ 121 & \text { Scrap } \\ 276 & \text { Waste } \\ 458 & \text { Paper } \\ 459 & \text { Cloth/Canvas } \\ 464 & \text { Plastic } \\ 465 & \text { Solvent } \\ 469 & \text { Wood }\end{array}$

521 Debris

527 Tritium, D20

633 Concrete

752 Radioactive

998 Asbestos 
TABLE 9

EQUIPMENT CODE (cont'd) (CATEGORY ORDER NUMERICAL LISTING)

\section{WASTE/RESTORATION (Cont'd)}

- Disposal

555 Vault

837 Low Level Waste Trench 842 Intermediate Level Waste /ELLT

- With Tritium Vault

- Disposal

838 Intermediate Level Waste Trench/GCD

843 Long lived Waste Vault

839 Intermediate Level NonTritium Waste Vault 907 Sanitary Landfill

840 Hazardous/Mixed Waste 964 Solid Waste Disposal. Vault Facility

841 Low Level Waste Vault 977 High Level Waste Trench 990 Interim Above Ground Storage

- Incineration/Compactor

\begin{tabular}{|c|c|c|c|c|}
\hline $\begin{array}{l}485 \\
693\end{array}$ & $\begin{array}{l}\text { Refractory } \\
\text { Beta Gamma }\end{array}$ & Incinerator & $\begin{array}{l}696 \\
701\end{array}$ & $\begin{array}{l}\text { Solid Burning } \\
\text { Spray/Spray } \\
\text { Chamber/Spray } \\
\text { Ouench }\end{array}$ \\
\hline & & & 702 & Ash Buildup \\
\hline $\begin{array}{l}694 \\
695\end{array}$ & $\begin{array}{l}\text { Burner } \\
\text { Solvent Bur }\end{array}$ & & 726 & Waste Compactor \\
\hline
\end{tabular}

- Restoration

863 Capping

865 Closure 
TABLE 9

EQUIPMENT CODE (cont'd) (CATEGORY ORDER NUMERICAL LISTING)

20 WASTE/RESTORATION (Cont'd)

- Restoration (cont'd)

$864^{\circ}$ Excavation

866 Waste Removal

867 Stabilization

997 Demolition/

Decommissioning

- Storage

831 Interim Mixed waste Treatment and storage

832 Interim Hazardous Waste Storage

833 Interim TRU Waste Storage/WCF

$835^{\circ}$ Organic Waste Storage

- Treatment

831 Interim Mixed Waste

836 Chemical/Industrial Treatment and Storage Waste Treatment

857. Dewatering Facility

950 Soils

\section{WORKS ENGINEERING}

Generic Terms

$\begin{array}{ll}075 & \text { Weld } \\ 202 & \text { Alignment } \\ 209 & \text { Hand Tools } \\ 273 & \text { Band Saw } \\ 291 & \text { Cutting } \\ 407 & \text { Machining } \\ 412 & \text { Welding } \\ 419 & \text { Dies }\end{array}$


TABLE 9

EQUIPMENT CODE (cont'd)

(CATEGORY ORDER NUMERICAL LISTING)

21. WORKS ENGINEERING (Cont'd)

Generic Terms (cont'd)

432 Repairs

454 Maintenance Operation

550 Drill/Drilling

552 Install/Replace/Remove

541 Maintenance Room

658 Tool

675. Robot

738 Pipe Cutter

763 Preventative Maintenance

769 Digging/Chipping

789 Welder

900 Predictive Maintenance

- Work Package

259 Locked Out

260 Closed Vessel Entry

271 Not locked Out or Tagged Out

771 Temporary Piping

809 Lock and Tag Violation

823 Work Order Deficiency

- Design

058 Design

233 Structural Component

252 Dimensions 
TABLE 10

\section{APPLICATIONS OF WASTE MANAGEMENT FAULT TREE DATABANK}

- Failure Rate Data

- Equipment Breakdown Histories

- Generic Incident Histories

- Data For Systems Analyses and Safety Analysis Reports

- Dates of Specific Events

- Consequences of Incidents

- Data for Design Studies

- Data for Quality Assurance Studies

- Trend Analyses

- Data for Project Justification

- Data for Process Hazards Analysis

- Training

- Process Problem Solving

- Management Decision Data

- Studies of Effectiveness of Administrative Controls

- Incident Audit

- Data for Reliability Studies

- Reference to Source Documents 